Linköping studies in science and technology. Licentiate Thesis No. 1838

\title{
On Informative Path \\ Planning for Tracking \\ and Surveillance
}

Per Boström-Rost

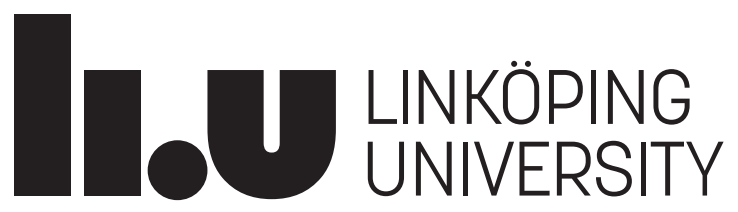




\section{On Informative Path Planning for Tracking and Surveillance}

\section{Per Boström-Rost}


This is a Swedish Licentiate's Thesis.

Swedish postgraduate education leads to a Doctor's degree and/or a Licentiate's degree.

A Doctor's Degree comprises 240 ECTS credits (4 years of full-time studies).

A Licentiate's degree comprises 120 ECTS credits,

of which at least 60 ECTS credits constitute a Licentiate's thesis.

Linköping studies in science and technology. Licentiate Thesis

No. 1838

On Informative Path Planning for Tracking and Surveillance

Per Boström-Rost

per.bostrom-rost@liu.se

ww. control.isy.liu.se

Department of Electrical Engineering

Linköping University

SE-581 83 Linköping

Sweden

ISBN 978-91-7685-075-6 ISSN 0280-7971

Copyright (C) 2019 Per Boström-Rost

Printed by LiU-Tryck, Linköping, Sweden 2019 



\section{Abstract}

This thesis studies a class of sensor management problems called informative path planning (IPP). Sensor management refers to the problem of optimizing control inputs for sensor systems in dynamic environments in order to achieve operational objectives. The problems are commonly formulated as stochastic optimal control problems, where to objective is to maximize the information gained from future measurements. In IPP, the control inputs affect the movement of the sensor platforms, and the goal is to compute trajectories from where the sensors can obtain measurements that maximize the estimation performance. The core challenge lies in making decisions based on the predicted utility of future measurements.

In linear Gaussian settings, the estimation performance is independent of the actual measurements. This means that IPP becomes a deterministic optimal control problem, for which standard numerical optimization techniques can be applied. This is exploited in the first part of this thesis. A surveillance application is considered, where a mobile sensor is gathering information about features of interest while avoiding being tracked by an adversarial observer. The problem is formulated as an optimization problem that allows for a trade-off between informativeness and stealth. We formulate a theorem that makes it possible to reformulate a class of nonconvex optimization problems with matrix-valued variables as convex optimization problems. This theorem is then used to prove that the seemingly intractable IPP problem can be solved to global optimality using off-the-shelf optimization tools.

The second part of this thesis considers tracking of a maneuvering target using a mobile sensor with limited field of view. The problem is formulated as an IPP problem, where the goal is to generate a sensor trajectory that maximizes the expected tracking performance, captured by a measure of the covariance matrix of the target state estimate. When the measurements are nonlinear functions of the target state, the tracking performance depends on the actual measurements, which depend on the target's trajectory. Since these are unavailable in the planning stage, the problem becomes a stochastic optimal control problem. An approximation of the problem based on deterministic sampling of the distribution of the predicted target trajectory is proposed. It is demonstrated in a simulation study that the proposed method significantly increases the tracking performance compared to a conventional approach that neglects the uncertainty in the future target trajectory. 



\section{Populärvetenskaplig sammanfattning}

Flygplan har varit en del av vårt samhälle i över hundra år. De första flygplanen var svårmanövrerade, vilket innebar att dåtidens piloter fick lägga en stor del av sin tid och kraft på att hålla planet i luften. Under åren har dock flygplanens styrsystem förbättrats avsevärt, vilket har möjliggjort för piloterna att utföra andra uppgifter utöver att styra dem. Som en följd av detta har allt fler sensorer installerats i flygplanen, vilket ger piloterna mer information om omvärlden. Även sensorerna måste dock styras, och numera lägger piloterna mer tid på att styra sensorer än att styra själva flygplanet.

Många sensorer som tidigare styrts för hand börjar nu bli alltför komplexa för att hanteras manuellt. Det här gör att behovet av automatiserad sensorstyrning (eng. sensor management) blir allt större. Den här avhandlingen fokuserar på en del av automatiserad sensorstyrning som ofta kallas informationbaserad ruttplanering (eng. informative path planning). Målet är att styra de rörliga plattformar som bär sensorerna så att mätningarna ger så mycket användbar information som möjligt.

Att automatiskt optimera styrsignaler till ett sensorsystem är en komplicerad process som görs i flera steg. Först används de mätningar som redan gjorts för att skatta tillståndet, vilket till exempel kan representera positionen och hastigheten, för ett visst objekt. Den här tillståndsskattningen används sedan för att prediktera vilka mätningar som sensorn kommer att kunna samla in i framtiden om en viss styrsignalssekvens används. Genom att använda de predikterade mätningarna går det att förutsäga hur tillståndsskattningen kommer att utvecklas, och vi kan därigenom välja styrsignalssekvensen på ett sådant sätt att kvaliteten på tillståndsskattningen blir så bra som möjligt.

Matematisk optimering används ofta för att formulera problem inom informationbaserad ruttplanering. Optimeringsproblemen är dock i allmänhet svåra att lösa, och även om man lyckas beräkna en rutt för sensorplattformen är det svårt att garantera att det inte finns en annan rutt som skulle vara ännu bättre. I den första delen av den här avhandlingen presenteras ett sätt att omformulera optimeringsproblemen så att de bästa möjliga rutterna går att beräkna. Omformuleringen går även att tillämpa i de fall där sensorplattformen behöver undvika att upptäckas av andra sensorer i området. Det här resultatet kan till exempel vara användbart när andra ruttplaneringsmetoder ska utvärderas.

Den andra delen av den här avhandlingen handlar om hur osäkerheter i optimeringsproblem inom informationbaserad ruttplanering ska hanteras. Scenariot som studeras är ett målföljningsscenario, där en rörlig sensor ska styras på ett sådant sätt att ett manövrerande objekt hålls inom sensorns synfält. En svårighet med detta är att sensorn måste prediktera hur objektet kommer att röra sig i framtiden. Den här prediktionen kan göras på flera sätt. Tidigare metoder för liknande problem har bara tagit hänsyn till den mest sannolika manövern. I det här arbetet presenteras en ny metod, som tar hänsyn till osäkerheten i objektets framtida rörelser genom att inkludera flera potentiella manövrar i beräkningarna. En simuleringsstudie visar på en signifikant förbättring av målföljningsprestandan när den nya metoden används jämfört med tidigare metoder. 



\section{Acknowledgments}

I am sincerely grateful to my supervisor, Assoc. Prof. Gustaf Hendeby, and my cosupervisor Assoc. Prof. Daniel Axehill. Your guidance and support during the last couple of years have been nothing but amazing. Thank you for all the feedback, encouragement, and for always being available for discussions.

I would like to thank Prof. Fredrik Gustafsson and Prof. Svante Gunnarsson for letting me join the Automatic Control group. I would also like to thank Prof. Svante Gunnarsson and Assoc. Prof. Martin Enqvist for maintaining a friendly and professional work environment, and Ninna Stensgård for taking care of all practicalities.

Dr. Per-Johan Nordlund, Lic. Oskar Ljungqvist, M.Sc. Kristoffer Bergman, M.Sc. Erik Hedberg, Lic. Martin Lindfors, and Dr. Jonatan Olofsson have proofread all or parts of this thesis. All your comments and suggestions are much appreciated. Thank you!

I would like to thank all my colleagues at the Automatic Control group for making this a great place to work. Special thanks to Kristoffer Bergman for all the quality time we have spent in our office. Thanks to the man with the confidence, Oskar Ljungqvist, for explaining how things should be done. Thank you Andreas Bergström, Angela Fontan, Erik Hedberg, Du Ho, Ylva Jung, Parinaz Kasebzadeh, Martin Lindfors, Jonas Linder, Gustav Lindmark, Magnus Malmström, Isak Nielsen, Shervin Parvini Ahmadi, Kamiar Radnosrati, Zoran Sjanic, Martin Skoglund, Clas Veibäck, Farnaz Adib Yaghmaie and everyone else for all the great moments during fika breaks and other occasions.

This work was supported by the Wallenberg AI, Autonomous Systems and Software Program (WASP) funded by the Knut and Alice Wallenberg Foundation. Their funding is gratefully acknowledged. WASP also introduced me to a large number of inspiring people. Thanks to all the students in AS batch 1 for the good times during conferences and trips. Thanks also go to Lars Pääjärvi at Saab Aeronautics for giving me the opportunity to start this journey.

Many thanks to my family and friends for your love and support. Last but not least, I would like to say a few words to my wonderful wife Emma. I cannot thank you enough for all your support and encouragement. I love you with all my heart.

Linköping, May 2019 Per Boström-Rost 



\section{Contents}

Notation

xiii

1 Introduction 1

1.1 Background and motivation . . . . . . . . . . . . . . 1

1.2 Research questions . . . . . . . . . . . . . . . . 3

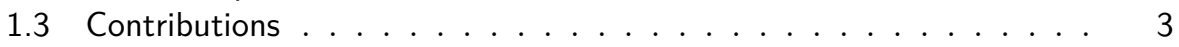

1.4 Thesis outline . . . . . . . . . . . . . . . . . 4

2 Background 7

2.1 State estimation $\ldots \ldots \ldots \ldots \ldots \ldots \ldots \ldots \ldots$

2.1.1 State-space models . . . . . . . . . . . . . . . . 7

2.1 .2 Bayesian filtering . . . . . . . . . . . . . . . 8

2.1 .3 Kalman filter . . . . . . . . . . . . . . . . 9

2.1 .4 Information filter . . . . . . . . . . . . . . 12

2.1 .5 Information measures . . . . . . . . . . . . . . . . . 14

2.2 Mathematical optimization . . . . . . . . . . . . . . . . . 15

2.2 .1 Convex optimization . . . . . . . . . . . . . . . . . 16

2.2 .2 Nonlinear optimization . . . . . . . . . . . . . . . . 17

2.3 Optimal control . . . . . . . . . . . . . . . . . 23

2.3.1 Deterministic optimal control . . . . . . . . . . . . . 23

2.3 .2 Stochastic optimal control . . . . . . . . . . . . . . . . 24

2.3 .3 Optimal motion planning . . . . . . . . . . . . . 27

2.3 .4 Informative path planning . . . . . . . . . . . . 29

3 Informative path planning in linear Gaussian settings 33

3.1 Separation principle . . . . . . . . . . . . . . . 33

3.2 Semidefinite program formulation . . . . . . . . . 36

3.2.1 Reformulation of nonlinear equality constraints . . . . . . 36

3.2.2 Application to informative path planning . . . . . . . 39

3.3 Informative path planning for surveillance . . . . . . . . . . 42

3.3.1 Problem formulation . . . . . . . . . . . . . . . . 43

3.3.2 Reformulation to mixed-binary semidefinite program . . . . 46

3.3.3 Numerical illustrations . . . . . . . . . . . . . . . . . . 47 
3.4 Avoiding adversarial observers ................. 50

3.4.1 Problem formulation ... . . . . . . . . . . . 50

3.4.2 Computing globally optimal solutions . . . . . . . . . . . 52

3.4.3 Stealthy informative path planning .......... 55

3.5 Summary ...................... 57

4 Informative path planning for active target tracking $\quad 59$

4.1 Problem formulation .................. 59

4.1.1 Motion and measurement models . . . . . . . . . . . . 59

4.1.2 Information representation . . . . . . . . . . . 60

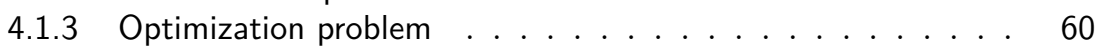

4.2 Approximations ...................... 61

4.2.1 Baseline approach . . . . . . . . . . . . . 6 62

4.2.2 Proposed approach . . . . . . . . . . . . . . 62

4.3 Graph search algorithm .................. . . . . . . 64

4.3.1 Search tree generation ............... . . . 64

4.3.2 Pruning strategies . . . . . . . . . . . . . . . 66

4.3.3 Resulting algorithm . . . . . . . . . . . . . 67

4.4 Simulation study . . . . . . . . . . . . . . . . . . . 69

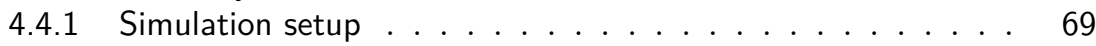

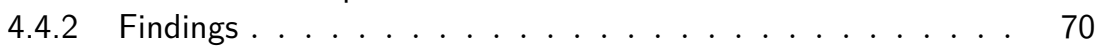

4.5 Summary . . . . . . . . . . . . . . . . . . 78

5 Concluding remarks $\quad \mathbf{7 9}$

5.1 Conclusions . . . . . . . . . . . . . . . . . . . . 79

5.2 Future work . . . . . . . . . . . . . . . . 80

$\begin{array}{lr}\text { Bibliography } & 81\end{array}$ 
Notation

Sets, spaces, and subspaces

\begin{tabular}{cl}
\hline Notation & Meaning \\
\hline $\mathbf{R}$ & Real numbers \\
$\mathbf{R}^{n}$ & Real $n$-vectors $(n \times 1$ matrices $)$ \\
$\mathbf{R}^{m \times n}$ & Real $m \times n$ matrices \\
$\mathbf{Z}$ & Integers \\
$\mathbf{Z}^{n}$ & Integer $n$-vectors \\
$\mathbf{S}^{n}$ & Symmetric $n \times n$ matrices \\
$\mathbf{S}_{+}^{n}$ & Symmetric positive semidefinite $n \times n$ matrices \\
$\mathbf{S}_{++}^{n}$ & Symmetric positive definite $n \times n$ matrices \\
{$[a, b]$} & Interval of real numbers $x$ such that $a \leq x \leq b$ \\
$a: b$ & Interval of integers $x$ such that $a \leq x \leq b$ \\
$\{a, b, \ldots\}$ & Discrete set of elements $a, b, \ldots$ \\
\hline
\end{tabular}

Symbols, operators, and functions

\begin{tabular}{cl}
\hline Notation & Meaning \\
\hline$I$ & Identity matrix \\
$X^{\top}, x^{\top}$ & Transpose of matrix $X$ or vector $x$ \\
$\operatorname{tr} X$ & Trace of matrix $X$ \\
$\lambda_{i}(X)$ & ith largest eigenvalue of matrix $X$ \\
$\lambda_{\max }(X)$ & Largest eigenvalue of matrix $X, \lambda_{1}(X)$ \\
$\operatorname{diag}(x)$ & Diagonal matrix with diagonal entries $x_{1}, \ldots, x_{n}$ \\
blkdiag $(X, Y, \ldots)$ & Block diagonal matrix with diagonal blocks $X, Y, \ldots$ \\
$X \succeq 0$ & Matrix $X$ is positive semidefinite \\
$X \succ 0$ & Matrix $X$ is positive definite \\
$x \succeq 0$ & All components of vector $x$ are nonnegative \\
$x \succ 0$ & All components of vector $x$ are positive \\
$f: A \rightarrow B$ & Function $f$ maps dom $f \subseteq A$ into $B$ \\
$\|x\|_{2}$ & Euclidean norm of vector $x$ \\
$\operatorname{dom} f$ & Domain of function $f$ \\
\hline &
\end{tabular}




\section{Symbols, operators, and functions, cont'd}

\begin{tabular}{cl}
\hline Notation & Meaning \\
\hline $\arg \max$ & Maximizing argument \\
$\arg \min$ & Minimizing argument \\
$\mathbf{E} x$ & Expected value of random variable $x$ \\
$\operatorname{cov} x$ & Covariance of random variable $x$ \\
$x \sim y$ & $x$ is distributed as $y$ \\
$\mathcal{N}(\mu, \Sigma)$ & Gaussian distribution with mean $\mu$ and covariance $\Sigma$ \\
$\mathcal{N}(x \mid \mu, \Sigma)$ & Gaussian PDF for $x$ with mean $\mu$ and covariance $\Sigma$ \\
$p(x)$ & Probability density function $($ PDF) of random variable $x$ \\
$p(x \mid y)$ & Conditional PDF of random variable $x$ given $y$ \\
$x_{k}$ & State at time $k$ \\
$x_{1: k}$ & Set of states from time 1 to $k$ \\
$s_{k}$ & Sensor state at time $k$ \\
$s_{1: k}$ & Set of sensor states from time 1 to $k$ \\
$u_{k}$ & Control input at time $k$ \\
$y_{k}$ & Measurement at time $k$ \\
$y_{1: k}$ & Set of measurements from time 1 to $k$ \\
$\hat{x}_{k \mid k}$ & Estimate of $x_{k}$ given measurements $y_{1: k}$ \\
$P_{k \mid k}$ & Covariance matrix at time $k$ given measurements $y_{1: k}$ \\
$\mathcal{I}_{k \mid k}$ & Information matrix at time $k$ given measurements $y_{1: k}$ \\
\hline &
\end{tabular}

\section{Acronyms and abbreviations}

\begin{tabular}{cl}
\hline Abbreviation & Meaning \\
\hline CEC & Certainty-equivalent control \\
EIF & Extended information filter \\
EKF & Extended Kalman filter \\
IF & Information filter \\
IPP & Informative path planning \\
KF & Kalman filter \\
MPC & Model predictive control \\
PDF & Probability density function \\
RIG & Rapidly exploring information gathering \\
RRT & Rapidly exploring random tree \\
RHC & Receding horizon control \\
RMSE & Root mean square error \\
SDP & Semidefinite program
\end{tabular}




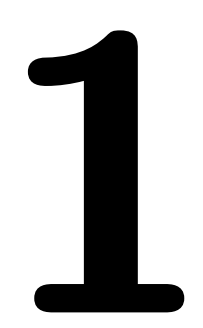

\section{Introduction}

Modern sensing systems often include several controllable operating modes and parameters. Sensor management, the problem of selecting the control inputs for this type of systems, is the topic of this thesis. The focus is on controlling the movement of the platforms that carry the sensors, a problem often referred to as informative path planning. This introductory chapter gives an overview of the concept of sensor management, lists the contributions, and outlines the content of the thesis.

\subsection{Background and motivation}

In the early days of aviation, controlling the aircraft was a challenging task for the pilot. Little time was available for anything aside from keeping the aircraft in the air. Over time, the capabilities of flight control systems have improved significantly, allowing pilots to perform more tasks than just controlling the aircraft while airborne. To enable this, an increasing number of sensors are being mounted on the aircraft to provide the pilots with information about the surroundings. As a result, a major part of the pilot's workload has shifted from controlling the aircraft to controlling the sensors. In a way, the pilots have become sensor operators.

An increasing number of sensors that traditionally have been controlled manually are now becoming too complex for a human to operate. This has led to the need for sensor management, which refers to the automation of sensor control systems, i.e., coordination of sensors in dynamic environments in order to achieve operational objectives.

With recent advances in remotely piloted aircraft technology, the sensor operators are no longer necessarily controlling sensors carried by a single platform. Modern sensing systems can consist of a large number of sophisticated sensors with many operating modes and functions. These can be distributed over several 

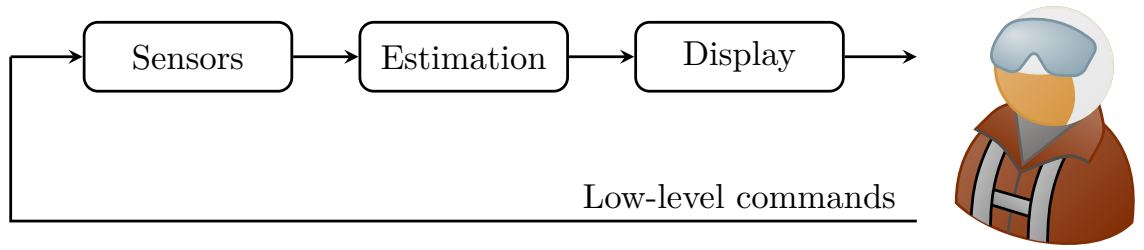

Operator

(a) Classical control of a sensing system
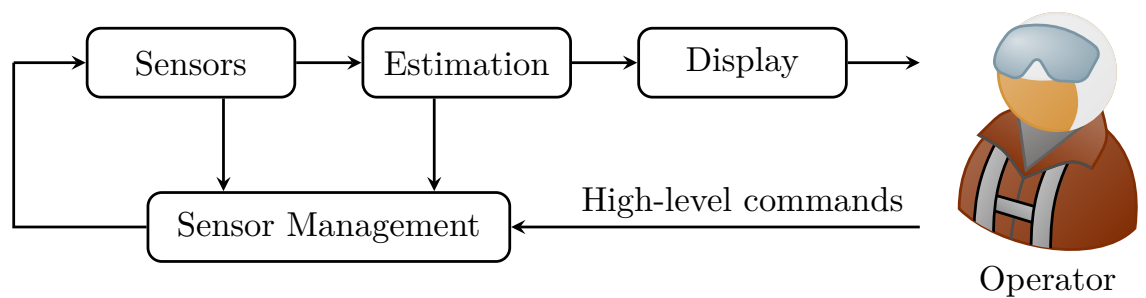

(b) Sensing system with sensor management component

Figure 1.1: Including a sensor management component in a sensing system reduces the workload of the operator.

different platforms with varying levels of communication capabilities. This means that the workload of the human operators that are controlling such systems is increasing rapidly. A sensor management system can reduce this workload. One of the main applications of sensor management is target tracking [6, 14, 27], where the sensor management problem corresponds to "directing the right sensor on the right platform to the right target at the right time" [47], but it can also be used for search and rescue [37], area exploration [75], and environmental monitoring $[22,48,62,66]$.

The authors of [14] argue that the significance of sensor management becomes clearer when considering the role of the operator in sensing systems with and without sensor management. The control loops of sensing systems with and without sensor management are illustrated in Figure 1.1. In a system that lacks sensor management, the operator acts as the controller and sends low-level commands to the sensors. If a sensor management component is available, it computes and sends low-level commands to the sensors based on high-level commands from the operator and the current situation at hand. According to [14], sensor management thus yields the following benefits:

- Reduced operator workload. Since the sensor management handles the lowlevel commands to the sensor, the operator can focus on the operational objectives of the sensing system rather than the details of its operation.

- More information available for decision making. A sensor management com- 
ponent can use all available information when deciding which low-level commands to use, while an operator is limited to the information presented on the displays.

- Faster control loops. An automated inner control loop allows for faster adaptation to changing conditions than one which involves the operator.

Sensor management has the potential of improving the performance of sensing systems and significantly decreasing the workload of their operators. Optimizing future sensor commands requires making decisions based on measurements that need to be predicted. Thus, sensor management is a research area in the intersection of estimation and control.

This thesis considers informative path planning (IPP), which is a subclass of sensor management. In informative path planning, the control inputs affect the movement of the sensor platforms. The goal is to compute trajectories from where the sensors can obtain measurements in order to accurately estimate the state of some system of interest. In general, the performance of state estimation methods depends on the actual measurements that are obtained. This is a complicating factor for informative path planning, as the objective function hence depends on future measurements that are unavailable at the time of planning. Due to this uncertainty, the informative path planning problem is a stochastic optimal control problem.

\subsection{Research questions}

The aim of this thesis is to provide answers to the following research questions:

- Which guarantees on the optimality of a sensor trajectory computed by an informative path planning method can be provided?

- How can a wish to avoid being observed be included in the informative path planning problem?

- How can the uncertainty in predictions of future measurements be handled?

\subsection{Contributions}

The main contributions of this thesis are presented in chapters 3 and 4 and are based on the following list of publications.

The first publication considers global optimization for informative path planning in linear Gaussian settings:

Per Boström-Rost, Daniel Axehill, and Gustaf Hendeby. On global optimization for informative path planning. IEEE Control Systems Letters, 2(4):833-838, 2018. ${ }^{1}$

\footnotetext{
${ }^{1}$ The contents of this paper were also selected for presentation at the $57^{\text {th }}$ IEEE Conference on Decision and Control, Miami Beach, FL, USA, 2018.
} 
The main contribution of this publication is a theorem that states equivalence between a convex and a nonconvex problem. The theorem can be used to reformulate a seemingly intractable informative path planning problem as a problem that can be solved to global optimality using off-the-shelf optimization tools based on branch and bound. This makes up the first part of Chapter 3.

The second part of Chapter 3 is made up of the following manuscript:

Per Boström-Rost, Daniel Axehill, and Gustaf Hendeby. Informative path planning in the presence of adversarial observers. Accepted for publication in Proceedings of the International Conference on Information Fusion (FUSION), Ottawa, Canada, 2019.

This contribution considers informative path planning in scenarios where the sensor platform has to avoid being tracked by an adversarial observer. By applying the theorem from the first contribution, it is shown that the considered problem can be solved to global optimality.

Chapter 4 is an edited version of the publication:

Per Boström-Rost, Daniel Axehill, and Gustaf Hendeby. Informative path planning for active tracking of agile targets. In Proceedings of IEEE Aerospace Conference, Big Sky, MT, USA, 2019.

This contribution deals with stochasticity in informative path planning problems. An active target tracking problem is solved by a new method that handles the uncertainty by considering multiple candidate target trajectories in parallel. The method is shown to outperform a standard method for informative path planning.

In all of the listed contributions, the author of this thesis has been the main driving force in the research, evaluations, and writing. The co-authors have contributed through technical discussions and by improving the manuscripts.

\subsection{Thesis outline}

This thesis is organized as follows.

- Chapter 1 (this chapter) introduces the research problem and lists the contributions of this thesis.

- Chapter 2 outlines the theoretical foundation of sensor management and informative path planning. It gives an introduction to state estimation, mathematical optimization, and optimal control theory.

- Chapter 3 is based on $[15,16]$ and presents results on informative path planning for surveillance and environmental monitoring applications. Assuming that the systems are linear and Gaussian, it is shown that the considered informative path planning problems are deterministic and can be solved to global optimality. 
- Chapter 4 is based on [17] and considers informative path planning for target tracking. Due to a nonlinear relation between the state of the target and the measurements, the planning problem becomes a stochastic optimal control problem. A deterministic approximation is proposed and evaluated in a simulation study.

- Chapter 5 concludes the thesis and gives some suggestions for future work. 



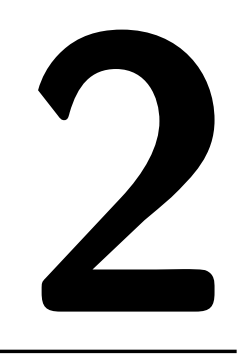

\section{Background}

This chapter provides the background theory that is utilized in this thesis. Section 2.1 gives an overview of statistical models of dynamical systems and methods to estimate the corresponding states. Section 2.2 briefly outlines the theory of mathematical optimization, and Section 2.3 describes the subject of optimal control, in which informative path planning belongs.

\subsection{State estimation}

This section provides an introduction to Bayesian state estimation, which considers the problem of inferring the state of an observed system from uncertain, noisy observations.

\subsubsection{State-space models}

A state-space model $[34,76]$ is a set of equations that characterize the evolution of a dynamical system and the relation between the state of the system and available measurements. One of the most general formulations of a state-space model is a probabilistic state-space model [67], which can be described by the conditional probability distributions:

$$
\begin{aligned}
x_{0} & \sim p\left(x_{0}\right), \\
x_{k+1} \mid x_{k} & \sim p\left(x_{k+1} \mid x_{k}\right), \\
y_{k} \mid x_{k} & \sim p\left(y_{k} \mid x_{k}\right),
\end{aligned}
$$

where $x_{k}$ is the state of the system and $y_{k}$ is a measurement, both at time index $k$. The distribution $p\left(x_{k+1} \mid x_{k}\right)$ is a state transition model that describes the stochastic dynamics of the system, the measurement likelihood function $p\left(y_{k} \mid x_{k}\right)$ 
describes the distribution of measurements given the state, and $p\left(x_{0}\right)$ is the probability distribution of the initial state of the system.

In state-space modeling, it is assumed that the system is Markovian, i.e., the state $x_{k+1}$ given $x_{k}$ is assumed to be conditionally independent of the earlier states and measurements, and the measurement $y_{k}$ depends only on the state $x_{k}$ :

$$
\begin{aligned}
p\left(x_{k+1} \mid x_{1: k}, y_{1: k}\right) & =p\left(x_{k+1} \mid x_{k}\right), \\
p\left(y_{k} \mid x_{1: k}, y_{1: k-1}\right) & =p\left(y_{k} \mid x_{k}\right) .
\end{aligned}
$$

Here, $x_{1: k}$ is the ordered set of states up until and including time $k$ and $y_{1: k}$ is defined analogously for the measurements. Due to the Markovian assumption, the state $x_{k}$ holds all information about the history of a system to predict its future behavior [76].

Instead of using conditional distributions to describe a state-space model, it can often be described by the functional representation

$$
\begin{aligned}
x_{k+1} & =f\left(x_{k}, w_{k}\right), \\
y_{k} & =h\left(x_{k}, e_{k}\right),
\end{aligned}
$$

where the state transition function $f$ and measurement function $h$ are general nonlinear functions, and $w_{k}$ and $e_{k}$ are the process noise and measurement noise, respectively. The noise terms $w_{k}$ and $e_{k}$, and the initial state $x_{0}$ are assumed to be mutually independent and described by probability distributions.

The general model (2.3) can be specialized by imposing constraints on the functions and distributions involved. An important special case is the linear statespace model with additive noise:

$$
\begin{aligned}
x_{k+1} & =F x_{k}+G w_{k}, \\
y_{k} & =H x_{k}+e_{k},
\end{aligned}
$$

where the state transition matrix $F$ and measurement matrix $H$ are independent of the state $x_{k}$. If the process noise and measurement noise are Gaussian distributed, (2.4) is referred to as a linear Gaussian state-space model [76].

\subsubsection{Bayesian filtering}

Recursive Bayesian estimation, or Bayesian filtering, is a probabilistic approach for estimating the posterior distribution $p\left(x_{k} \mid y_{1: k}\right)$ of the state $x_{k}$ given measurements $y_{1: k}$, and knowledge of the state transition function and measurement model. This section gives a brief introduction to the subject. For more details, see e.g. [67].

With the Markovian assumption, the Bayesian filtering problem for the probabilistic state-space model (2.1) can be solved by recursively computing the following distributions:

$$
\begin{aligned}
p\left(x_{k} \mid y_{1: k}\right) & =\frac{p\left(y_{k} \mid x_{k}\right) p\left(x_{k} \mid y_{1: k-1}\right)}{p\left(y_{k} \mid y_{1: k-1}\right)}, \\
p\left(x_{k+1} \mid y_{1: k}\right) & =\int p\left(x_{k+1} \mid x_{k}\right) p\left(x_{k} \mid y_{1: k}\right) d x_{k},
\end{aligned}
$$




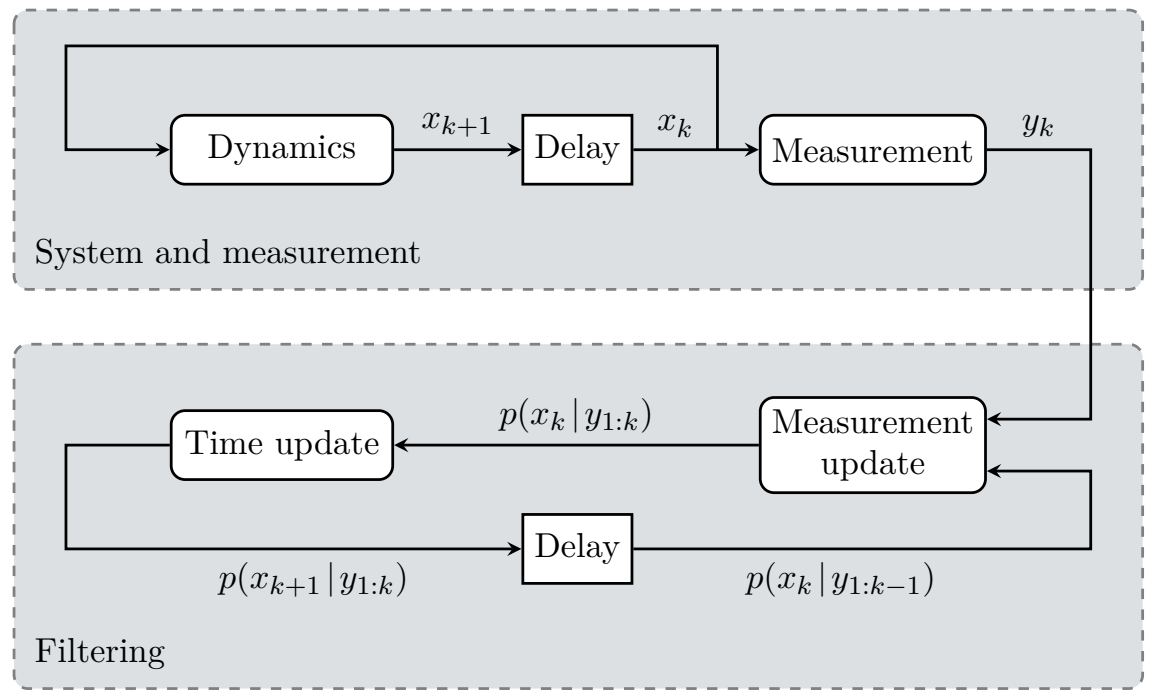

Figure 2.1: Bayesian filtering.

where the first equation is a measurement update in which the filtering distribution $p\left(x_{k} \mid y_{1: k}\right)$ is computed, and the second equation corresponds to computing the predictive distribution $p\left(x_{k+1} \mid y_{1: k}\right)$ in a time update. The normalization factor $p\left(y_{k} \mid y_{1: k-1}\right)$ is given by

$$
p\left(y_{k} \mid y_{1: k-1}\right)=\int p\left(y_{k} \mid x_{k}\right) p\left(x_{k} \mid y_{1: k-1}\right) d x_{k} .
$$

Figure 2.1 illustrates the two update steps of the Bayesian filter and its relation to the system dynamics and measurements. While the Bayesian recursion is theoretically appealing, the filtering distribution can in general not be computed in closed form, and analytical solutions exist only for a few special cases.

\subsubsection{Kalman filter}

In the special case of linear Gaussian systems, the well-known Kalman filter (KF) provides an analytical solution to the Bayesian filtering problem. The Kalman filter, which was first derived in [35], is briefly outlined in this section. For more in-depth treatments, the reader is referred to [34, 67].

Consider the linear state-space model (2.4), with white Gaussian process noise with mean $\mathbf{E} w_{k}=0$ and covariance $Q$, white Gaussian measurement noise with mean $\mathbf{E} e_{k}=0$ and covariance $R$, and the initial state $x_{0}$ Gaussian distributed with mean $\hat{x}_{0}$ and covariance $P_{0}$. The state transition model $(2.1 \mathrm{~b})$ and measurement likelihood function (2.1c) of the corresponding probabilistic state-space model are then Gaussian distributed according to

$$
\begin{aligned}
p\left(x_{k+1} \mid x_{k}\right) & =\mathcal{N}\left(x_{k+1} \mid F x_{k}, G Q G^{\top}\right), \\
p\left(y_{k} \mid x_{k}\right) & =\mathcal{N}\left(y_{k} \mid H x_{k}, R\right) .
\end{aligned}
$$


The Kalman filter is obtained by analytical evaluation of (2.5). Given the a priori state distribution $p\left(x_{k} \mid y_{1: k-1}\right)=\mathcal{N}\left(x_{k} \mid \hat{x}_{k \mid k-1}, P_{k \mid k-1}\right)$, where the index $k_{1} \mid k_{2}$ indicates estimate at time $k_{1}$ given measurements up until time $k_{2}$, the measurement update step (2.5a) reduces to

$$
\begin{aligned}
p\left(x_{k} \mid y_{1: k}\right) & =\frac{p\left(y_{k} \mid x_{k}\right) p\left(x_{k} \mid y_{1: k-1}\right)}{p\left(y_{k} \mid y_{1: k-1}\right)} \\
& =\mathcal{N}\left(x_{k} \mid \hat{x}_{k \mid k-1}+K_{k}\left(y_{k}-H \hat{x}_{k \mid k-1}\right), P_{k \mid k-1}-K_{k} H P_{k \mid k-1}\right) \\
& =\mathcal{N}\left(x_{k} \mid \hat{x}_{k \mid k}, P_{k \mid k}\right),
\end{aligned}
$$

where the matrix

$$
K_{k}=P_{k \mid k-1} H^{\top}\left(H P_{k \mid k-1} H^{\top}+R\right)^{-1}
$$

is called the Kalman gain. Similarly, since the state transition model is Gaussian, the time update step $(2.5 \mathrm{~b})$ reduces to

$$
\begin{aligned}
p\left(x_{k+1} \mid y_{1: k}\right) & =\int p\left(x_{k+1} \mid x_{k}\right) p\left(x_{k} \mid y_{1: k}\right) d x_{k} \\
& =\mathcal{N}\left(x_{k+1} \mid F \hat{x}_{k \mid k}, F P_{k \mid k} F^{\top}+G Q G^{\top}\right) \\
& =\mathcal{N}\left(x_{k+1} \mid \hat{x}_{k+1 \mid k}, P_{k+1 \mid k}\right) .
\end{aligned}
$$

Since both the predictive distribution and the filtering distribution are Gaussian, they are completely described by their corresponding means and covariance matrices. As outlined in Algorithm 2.1, these are the quantities that are propagated by the Kalman filter.

Note that the equations for the covariance matrices $P_{k \mid k}$ and $P_{k+1 \mid k}$ in the Kalman filter are both independent of the measurements $y_{1: k}$. Accordingly, the posterior covariance matrix can computed in advance, before any measurements have been obtained. This is an important property of the Kalman filter, which we will exploit in Chapter 3, where a measure of the resulting covariance matrix will be used to design sensing strategies.

In practical applications, nonlinearities often are present in either the dynamics or the measurement model and no analytical solution to the Bayesian filtering recursion can be computed. Hence, approximations are needed. A common idea is to approximate the true posterior distribution by a Gaussian with mean and covariance corresponding to those of $p\left(x_{k} \mid y_{1: k}\right)$. The extended Kalman filter (EKF) [31] does this by propagating estimates of the mean and covariance time by first linearizing the dynamics and measurement functions at the current state estimate, and then applying the Kalman filter equations in the measurement and time updates. In contrast to the Kalman filter, the covariance matrices computed by the EKF depend on the measurements since the nominal values used for linearization depend on the measurement values. Thus, computation of the resulting covariance matrix at design time is no longer possible. Algorithm 2.2 outlines the EKF for a general state-space model with additive white measurement noise, i.e.,

$$
\begin{aligned}
x_{k+1} & =f\left(x_{k}, w_{k}\right), \\
y_{k} & =h\left(x_{k}\right)+e_{k},
\end{aligned}
$$

where $\operatorname{cov} w_{k}=Q, \operatorname{cov} e_{k}=R$, and it is assumed that $\mathbf{E} w_{k}=0$ and $\mathbf{E} e_{k}=0$. 


\section{Algorithm 2.1: Kalman filter}

Input: Prior $x_{0} \sim p\left(x_{0}\right)$ and measurements $y_{0: N}$

Initialize:

$$
\begin{aligned}
& \hat{x}_{0 \mid-1}=\mathbf{E} x_{0} \\
& P_{0 \mid-1}=\operatorname{cov} x_{0}
\end{aligned}
$$

for $k=0: N$ do

\section{Measurement update:}

$$
\begin{aligned}
& K_{k}=P_{k \mid k-1} H^{\top}\left(H P_{k \mid k-1} H^{\top}+R\right)^{-1} \\
& \hat{x}_{k \mid k}=\hat{x}_{k \mid k-1}+K_{k}\left(y_{k}-H \hat{x}_{k \mid k-1}\right) \\
& P_{k \mid k}=P_{k \mid k-1}-K_{k} H P_{k \mid k-1}
\end{aligned}
$$

\section{Time update:}

end for

$$
\begin{aligned}
& \hat{x}_{k+1 \mid k}=F \hat{x}_{k \mid k} \\
& P_{k+1 \mid k}=F P_{k \mid k} F^{\top}+G Q G^{\top}
\end{aligned}
$$

Output: $\hat{x}_{k \mid k}, P_{k \mid k}, k=0, \ldots, N$

\section{Algorithm 2.2: Extended Kalman filter}

Input: Prior $x_{0} \sim p\left(x_{0}\right)$ and measurements $y_{0: N}$

Initialize:

$$
\begin{aligned}
& \hat{x}_{0 \mid-1}=\mathbf{E} x_{0} \\
& P_{0 \mid-1}=\operatorname{cov} x_{0}
\end{aligned}
$$

for $k=0: N$ do

\section{Measurement update:}

$$
\begin{gathered}
H_{k}=\left.\frac{\partial}{\partial x} h(x)\right|_{x=\hat{x}_{k \mid k-1}} \\
K_{k}=P_{k \mid k-1} H_{k}^{\top}\left(H_{k} P_{k \mid k-1} H_{k}^{\top}+R\right)^{-1} \\
\hat{x}_{k \mid k}=\hat{x}_{k \mid k-1}+K_{k}\left(y_{k}-h\left(\hat{x}_{k \mid k-1}\right)\right) \\
P_{k \mid k}=P_{k \mid k-1}-K_{k} H_{k} P_{k \mid k-1} \\
\text { Time update: } \\
F_{k}=\left.\frac{\partial}{\partial x} f(x, 0)\right|_{x=\hat{x}_{k \mid k}} \\
G_{k}=\left.\frac{\partial}{\partial w} f\left(\hat{x}_{k \mid k}, w\right)\right|_{w=0} \\
\hat{x}_{k+1 \mid k}=f\left(\hat{x}_{k \mid k}, 0\right) \\
P_{k+1 \mid k}=F_{k} P_{k \mid k} F_{k}^{\top}+G_{k} Q G_{k}^{\top}
\end{gathered}
$$

end for

Output: $\hat{x}_{k \mid k}, P_{k \mid k}, k=0, \ldots, N$ 


\subsubsection{Information filter}

An alternative formulation of the Kalman filter is the information filter (IF) [34]. Instead of maintaining the mean and covariance as in the Kalman filter, the information filter maintains an information state and an information matrix. The information matrix is the inverse of the covariance matrix $\mathcal{I}_{k}=P_{k}^{-1}$, and the information state is defined as $\iota_{k}=P_{k}^{-1} \hat{x}_{k}$.

Assuming that the measurement noise covariance matrix in the linear Gaussian state-space model is positive definite, and that $P_{k}^{-1}$ exists, the information filter can be derived from the Kalman filter equations in Algorithm 2.1. Using the Woodbury matrix identity [24], which states that for invertible matrices $A$ and $C$ and any matrices $U$ and $V$ of appropriate dimensions

$$
(A+U C V)^{-1}=A^{-1}-A^{-1} U\left(C^{-1}+V A^{-1} U\right)^{-1} V A^{-1},
$$

the measurement update step for the information matrix can be obtained as follows [34]:

$$
\begin{aligned}
\mathcal{I}_{k \mid k} & =P_{k \mid k}^{-1} \\
& =\left(P_{k \mid k-1}-K_{k} H P_{k \mid k-1}\right)^{-1} \\
& =\left(P_{k \mid k-1}-P_{k \mid k-1} H^{\top}\left(H P_{k \mid k-1} H^{\top}+R\right)^{-1} H P_{k \mid k-1}\right)^{-1} \\
& =P_{k \mid k-1}^{-1}+H^{\top} R^{-1} H \\
& =\mathcal{I}_{k \mid k-1}+H^{\top} R^{-1} H .
\end{aligned}
$$

Note that the posterior information matrix $\mathcal{I}_{k \mid k}$ is the sum of the prior information matrix $\mathcal{I}_{k \mid k-1}$ and the information contained in the new measurement, $H^{\top} R^{-1} H$. Equation (2.13), together with the fact that the Kalman gain can be rewritten as

$$
K_{k}=P_{k \mid k} H^{\top} R^{-1},
$$

lead to the following expression for the measurement update step for the information state [34]:

$$
\begin{aligned}
\iota_{k \mid k} & =P_{k \mid k}^{-1} \hat{x}_{k \mid k} \\
& =P_{k \mid k}^{-1}\left(\hat{x}_{k \mid k-1}+K_{k}\left(y_{k}-H \hat{x}_{k \mid k-1}\right)\right) \\
& =P_{k \mid k}^{-1}\left(\hat{x}_{k \mid k-1}+P_{k \mid k} H^{\top} R^{-1}\left(y_{k}-H \hat{x}_{k \mid k-1}\right)\right) \\
& =\underbrace{\left(P_{k \mid k}^{-1}-H_{k}^{\top} R^{-1} H_{k}\right) \hat{x}_{k \mid k-1}}_{=\iota_{k \mid k-1}}+H^{\top} R^{-1} y_{k} \\
& =\iota_{k \mid k-1}+H^{\top} R^{-1} y_{k} .
\end{aligned}
$$

The information filter is outlined in Algorithm 2.3. The change of variables from the Kalman filter results in a shift of computational complexity from the measurement update step to the time update step. Since information is additive, 


\section{Algorithm 2.3: Information filter}

Input: Prior $x_{0} \sim p\left(x_{0}\right)$ and measurements $y_{0: N}$

Initialize:

$$
\begin{gathered}
\mathcal{I}_{0 \mid-1}=\left(\operatorname{cov} x_{0}\right)^{-1} \\
\iota_{0 \mid-1}=\mathcal{I}_{0 \mid-1} \mathbf{E} x_{0} \\
\text { for } k=0: N \text { do }
\end{gathered}
$$

\section{Measurement update:}

\section{end for}

$$
\begin{aligned}
& \mathcal{I}_{k \mid k}=\mathcal{I}_{k \mid k-1}+H^{\top} R^{-1} H \\
& \iota_{k \mid k}=\iota_{k \mid k-1}+H^{\top} R^{-1} y_{k}
\end{aligned}
$$

\section{Time update:}

$$
\begin{aligned}
& \mathcal{I}_{k+1 \mid k}=\left(F_{k} \mathcal{I}_{k \mid k}^{-1} F_{k}^{\top}+G Q G^{\top}\right)^{-1} \\
& \iota_{k+1 \mid k}=\mathcal{I}_{k+1 \mid k} F_{k} \mathcal{I}_{k \mid k}^{-1} \iota_{k \mid k}
\end{aligned}
$$

Output: $\iota_{k \mid k}, \mathcal{I}_{k \mid k}, k=0, \ldots, N$

the measurement update step is cheaper in an information filter, but the time update step is cheaper in a Kalman filter. Both the time update step and measurement update step for the information matrix are independent of the actual measurement values, which means that the posterior information matrix can be recursively computed in advance, before any measurements have been obtained.

The extended information filter (EIF) [68] is an extension of the information filter that can handle nonlinear models as (2.11), in the same way that the EKF extends the Kalman filter to the nonlinear case. It is derived from the EKF by applying the change of variables $\mathcal{I}_{k}=P_{k}^{-1}$ and $\iota_{k}=P_{k}^{-1} \hat{x}_{k}$. The main difference compared to the linear information filter is in the measurement update step for the information state, which becomes

$$
\begin{aligned}
\iota_{k \mid k} & =P_{k \mid k}^{-1} \hat{x}_{k \mid k} \\
& =P_{k \mid k}^{-1}\left(\hat{x}_{k \mid k-1}+K_{k}\left(y_{k}-h\left(\hat{x}_{k \mid k-1}\right)\right)\right) \\
& =P_{k \mid k}^{-1}\left(\hat{x}_{k \mid k-1}+P_{k \mid k} H_{k}^{\top} R^{-1}\left(y_{k}-h_{k}\left(\hat{x}_{k \mid k-1}\right)\right)\right) \\
& =\left(P_{k \mid k}^{-1}-H_{k}^{\top} R^{-1} H_{k}+H_{k}^{\top} R^{-1} H_{k}\right) \hat{x}_{k \mid k-1}+H_{k}^{\top} R^{-1}\left(y_{k}-h_{k}\left(\hat{x}_{k \mid k-1}\right)\right) \\
& =\underbrace{\left(P_{k \mid k}^{-1}-H_{k}^{\top} R^{-1} H_{k}\right) \hat{x}_{k \mid k-1}}_{=\iota_{k \mid k-1}}+H_{k}^{\top} R^{-1}\left(y_{k}-h_{k}\left(\hat{x}_{k \mid k-1}\right)+H_{k} \hat{x}_{k \mid k-1}\right) \\
& =\iota_{k \mid k-1}+H_{k}^{\top} R^{-1}\left(y_{k}-h_{k}\left(\hat{x}_{k \mid k-1}\right)+H_{k} \hat{x}_{k \mid k-1}\right) .
\end{aligned}
$$

The extended information filter is outlined for the model (2.11) in Algorithm 2.4. 


\section{Algorithm 2.4: Extended information filter}

Input: Prior $x_{0} \sim p\left(x_{0}\right)$ and measurements $y_{0: N}$

Initialize:

$$
\begin{aligned}
& \mathcal{I}_{0 \mid-1}=\left(\operatorname{cov} x_{0}\right)^{-1} \\
& \iota_{0 \mid-1}=\mathcal{I}_{0 \mid-1} \mathbf{E} x_{0}
\end{aligned}
$$

for $k=0: N$ do

Measurement update:

$$
\begin{aligned}
& \hat{x}_{k \mid k-1}=\mathcal{I}_{k \mid k-1}^{-1} \iota_{k \mid k-1} \\
& H_{k}=\left.\frac{\partial}{\partial x} h(x)\right|_{x=\hat{x}_{k \mid k-1}} \\
& \mathcal{I}_{k \mid k}=\mathcal{I}_{k \mid k-1}+H_{k}^{\top} R^{-1} H_{k} \\
& \iota_{k \mid k}=\iota_{k \mid k-1}+H_{k}^{\top} R^{-1}\left(y_{k}-h_{k}\left(\hat{x}_{k \mid k-1}\right)+H_{k} \hat{x}_{k \mid k-1}\right)
\end{aligned}
$$

\section{Time update:}

$$
\begin{aligned}
& \hat{x}_{k \mid k}=\mathcal{I}_{k \mid k}^{-1} \iota_{k \mid k} \\
& F_{k}=\left.\frac{\partial}{\partial x} f(x, 0)\right|_{x=\hat{x}_{k \mid k}} \\
& G_{k}=\left.\frac{\partial}{\partial w} f\left(\hat{x}_{k \mid k}, w\right)\right|_{w=0} \\
& \mathcal{I}_{k+1 \mid k}=\left(F_{k} \mathcal{I}_{k \mid k}^{-1} F_{k}^{\top}+G Q G^{\top}\right)^{-1} \\
& \iota_{k+1 \mid k}=\mathcal{I}_{k+1 \mid k} f\left(\hat{x}_{k \mid k}, 0\right)
\end{aligned}
$$

end for

Output: $\iota_{k \mid k}, \mathcal{I}_{k \mid k}, k=0, \ldots, N$

\subsubsection{Information measures}

This section introduces a number of scalar measures for performance evaluation of state estimation methods. When using a Kalman filter or information filter, a measure based on the covariance matrix or information matrix can be used as an indication of the estimation performance. There are many different scalarizations that could be employed; [74] gives a thorough analysis of several alternatives. The use of scalar measures of covariance and information matrices occurs also in the field of experiment design [59], e.g., in the $A-, T-, D$ - and $E$-optimality criteria, which are introduced next.

\section{Trace}

Using the $A$-optimality criterion, the objective is to minimize the trace of the covariance matrix,

$$
\ell_{A}(P)=\operatorname{tr} P=\sum_{i=1}^{n} \lambda_{i}(P),
$$

where $\lambda_{i}(P)$ is the $i$ th largest eigenvalue of $P$ and $n$ is the number of rows in $P$. This results in minimizing the expected mean square error of the state estimate [74]. Note that the $A$-optimality criterion, i.e., minimizing the sum of the eigenvalues of the covariance matrix, is not equivalent to minimizing the negative trace of the 
information matrix, i.e., the $T$-optimality criterion,

$$
\ell_{T}(\mathcal{I})=-\operatorname{tr} \mathcal{I}=-\sum_{i=1}^{n} \lambda_{i}(\mathcal{I})=-\sum_{i=1}^{n} 1 / \lambda_{i}(P),
$$

which instead corresponds to minimizing the negative sum of the inverse eigenvalues of the covariance matrix.

\section{Determinant}

Using the $D$-optimality criterion, the objective is to maximize the determinant of the information matrix,

$$
\ell_{D}(\mathcal{I})=-\operatorname{det} \mathcal{I}=-\prod_{i=1}^{n} \lambda_{i}(\mathcal{I})
$$

The $D$-optimality criterion has an information-theoretic interpretation. The differential entropy [21], which is a measure of the uncertainty, is defined for a random variable $x$ with distribution $p(x)$ as:

$$
H(x)=-\mathbf{E} \log p(x)=-\int p(x) \log p(x) d x .
$$

If $x$ is an $n$-dimensional Gaussian distributed vector with covariance $P$, its differential entropy is given by

$$
H(x)=\frac{1}{2}(n \log (2 \pi e)+\log \operatorname{det} P)=-\frac{1}{2}(-n \log (2 \pi e)+\log \operatorname{det} \mathcal{I}) .
$$

Since the natural logarithm is a monotonically increasing function [18], this means that minimizing the $D$-optimality criterion is equivalent to minimizing the differential entropy in the Gaussian case.

\section{Eigenvalue}

Another example from experiment design is the $E$-optimality criterion, for which the maximum eigenvalue of the covariance matrix is minimized,

$$
\ell_{E}(P)=\lambda_{\max }(P) .
$$

This can be interpreted as minimizing the largest semi-axis of the confidence ellipsoid, or simply minimizing uncertainty in the most uncertain direction [74].

\subsection{Mathematical optimization}

This section introduces the concept of mathematical optimization and some methods for solving optimization problems. The notation and explanations are inspired by [18], which is an extensive resource on mathematical optimization. 
A general optimization problem can be written as

$$
\begin{array}{ll}
\underset{x}{\operatorname{minimize}} & f_{0}(x) \\
\text { subject to } & f_{i}(x) \leq 0, \quad i=1, \ldots, m \\
& h_{i}(x)=0, \quad i=1, \ldots, p,
\end{array}
$$

where $x \in \mathbf{R}^{n}$ is the decision variable and $f_{0}: \mathbf{R}^{n} \rightarrow \mathbf{R}$ is the objective function. The functions $f_{i}: \mathbf{R}^{n} \rightarrow \mathbf{R}, i=1, \ldots, m$, are denoted inequality constraint functions and the functions $h_{i}: \mathbf{R}^{n} \rightarrow \mathbf{R}, i=1, \ldots, p$, are called equality constraint functions. The goal is to compute a solution $x^{\star}$ that minimizes the objective function while belonging to the feasible set, defined by the set of points that satisfies all constraints of (2.23). A solution can either be a globally or a locally optimal solution. If $x^{\star}$ is a globally optimal solution, no other feasible point $z$ gives a smaller objective function value, i.e., $f_{0}(z) \geq f_{0}\left(x^{\star}\right)$ for any $z$ that satisfies $f_{i}(z) \leq 0, i=1, \ldots, m$, and $h_{i}(z)=0, i=1, \ldots, p$. A locally optimal solution is a point $x^{\star}$ at which the objective function value is smaller than at all other feasible points in a neighborhood of $x^{\star}$. The optimal value $p^{\star}$ of the problem (2.23) is defined as

$$
p^{\star}=\inf \left\{f_{0}(x) \mid f_{i}(x) \leq 0, i=1, \ldots, m, h_{i}(x)=0, i=1, \ldots, p\right\} .
$$

Two optimization problems are said to be equivalent if the optimal solution to one of the problems can be trivially computed from the solution to the other problem, and vice versa.

\subsubsection{Convex optimization}

This section introduces a class of optimization problems referred to as convex optimization problems. The following definitions will be needed:

Definition 2.1 (Convex set). A set $\mathcal{C}$ is a convex set if it holds that

$$
\theta x+(1-\theta) y \in \mathcal{C}
$$

for all $x, y \in \mathcal{C}$ and $\theta \in[0,1]$.

Definition 2.2 (Convex function). A function $f: \mathbf{R}^{n} \rightarrow \mathbf{R}$ is a convex function if $\operatorname{dom}(f)$ is a convex set and it satisfies

$$
f(\theta x+(1-\theta) y) \leq \theta f(x)+(1-\theta) f(y)
$$

for all $x, y \in \operatorname{dom}(f)$ and $\theta \in[0,1]$.

Having defined convex sets and functions, we can now characterize a convex optimization problem. We follow [18] and define a convex optimization problem as a special case of the general optimization problem that has the following properties:

- the objective function $f_{0}$ is convex,

- the inequality constraint functions $f_{i}$ are convex, 
- the equality constraint functions $h_{i}$ are affine.

A convex optimization problem can thus be formulated as

$$
\begin{array}{ll}
\underset{x}{\operatorname{minimize}} & f_{0}(x) \\
\text { subject to } & f_{i}(x) \leq 0, \quad i=1, \ldots, m \\
& a_{i}^{\top} x=b_{i}, \quad i=1, \ldots, p,
\end{array}
$$

where $f_{i}, i=0, \ldots, m$, are convex functions.

Convexity is an important concept in optimization. For convex optimization, there exist effective algorithms that can reliably and efficiently solve even large problems [18]. A fundamental property of convex optimization problems is that any locally optimal solution is also a globally optimal solution.

\section{Semidefinite programming}

Semidefinite programs (SDPs) are convex optimization problems that involve matrix inequalities [18]. Typically, the matrix inequality constrains all eigenvalues of a matrix-valued function of the decision variable to be non-positive, i.e., the resulting matrix is constrained to be negative semidefinite. Mathematically, an SDP problem can be formulated as

$$
\begin{array}{cl}
\underset{X}{\operatorname{minimize}} & f_{0}(X) \\
\text { subject to } & f_{i}(X) \preceq 0, \quad i=1, \ldots, m \\
& h_{i}(X)=0, \quad i=1, \ldots, p .
\end{array}
$$

Many problems in control and estimation can be formulated as SDPs [19].

\subsubsection{Nonlinear optimization}

An instance of the general optimization problem (2.23) is referred to as a nonlinear optimization problem if at least one of the constraint functions or the objective function is nonlinear [18]. An important subclass of nonlinear optimization problems is the previously discussed convex optimization problems, for which locally optimal solutions are also globally optimal. This is however not the case for nonlinear optimization problems in general. For many problems, it is difficult to verify if a locally optimal solution is in fact a globally optimal solution and finding the globally optimal solution can be extremely challenging [50]. Due to the difficulty of locating and verifying globally optimal solutions, much attention has been focused towards local optimization, i.e., computing locally optimal solutions. See e.g. $[7,12,50]$ for extensive textbooks on the subject. A solution that is obtained using local optimization might depend on the initial guess that was given to the optimization algorithm and there is no guarantee that it does not exist a different feasible point that produces a lower objective function value.

The procedure of locating and verifying the globally optimal solution to a general nonlinear optimization problem is called global optimization [18]. As mentioned earlier, this is in general a difficult task. However, for certain problem 
classes, global optimization techniques can be used to retrieve a global solution. Next, a class of such problems is introduced.

\section{Mixed-binary convex optimization problems}

In the preceding sections, all decision variables are real-valued. Mathematical optimization is however not restricted to only real-valued variables. An optimization problem that involves both real-valued variables $x \in \mathbf{R}^{n_{x}}$ and integer-valued variables $\delta \in \mathbf{Z}^{n_{\delta}}$ is referred to as a mixed-integer optimization problem. Any optimization problem of this type is a nonconvex problem, since the corresponding feasible set is a nonconvex set.

A subset of mixed-integer optimization is mixed-binary optimization, where all integer variables are constrained to be binary. A special case of mixed-binary problems is the mixed-binary convex problem, which is defined as

$$
\begin{array}{cl}
\underset{x, \delta}{\operatorname{minimize}} & f_{0}(x, \delta) \\
\text { subject to } & f_{i}(x, \delta) \leq 0, \quad i=1, \ldots, m \\
& h_{i}(x, \delta)=0, \quad i=1, \ldots, p \\
& \delta_{i} \in\{0,1\}, \quad i=1, \ldots, n_{\delta},
\end{array}
$$

where $x \in \mathbf{R}^{n_{x}}$ and $\delta \in\{0,1\}^{n_{\delta}}$ are the decision variables, the inequality constraint functions are convex, and the equality constraint functions are affine. The property that makes mixed-binary convex problems different from standard mixed-binary problems is that when the binary constraints $\delta_{i} \in\{0,1\}, i=1, \ldots, n_{\delta}$, are relaxed to interval constraints $\delta_{i} \in[0,1], i=1, \ldots, n_{\delta}$, the relaxed optimization problem,

$$
\begin{array}{cl}
\underset{x, \delta}{\operatorname{minimize}} & f_{0}(x, \delta) \\
\text { subject to } & f_{i}(x, \delta) \leq 0, \quad i=1, \ldots, m \\
& h_{i}(x, \delta)=0, \quad i=1, \ldots, p \\
& \delta_{i} \in[0,1], \quad i=1, \ldots, n_{\delta},
\end{array}
$$

is convex and can be solved efficiently to global optimality. The problem (2.30) is referred to as a convex relaxation of (2.29). The two problems have the same objective function and the feasible set of (2.29) is a subset of the feasible set of (2.30). It is thus possible to draw the following conclusions regarding the original problem by solving the relaxed one:

- The optimal value of the relaxed problem is a lower bound on the optimal value of the original problem.

- If the relaxed problem is infeasible, then so is the original problem.

- If an optimal solution to the relaxed problem is feasible in the original one, then it must also be an optimal solution to the original problem. 


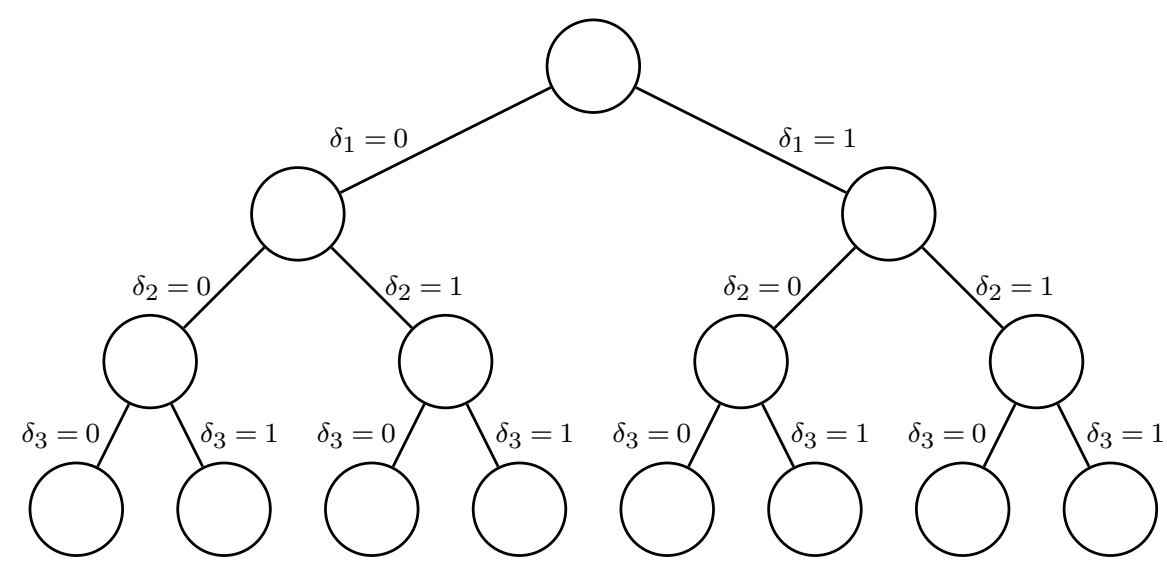

Figure 2.2: Example of a binary search tree with three binary variables.

The most straightforward approach to compute an optimal solution to a mixedbinary convex problem is to perform an exhaustive search, in which a convex problem is solved for each possible combination of the binary variables. The globally optimal solution is then the point that is feasible with respect to the constraints and gives the smallest objective function value. Since the number of convex problems that need to be solved increases exponentially with the number of binary variables, the computational cost of performing an exhaustive search quickly becomes overwhelming. Several successful approaches to solve mixed-binary problems more efficiently have been proposed [23], and the next section introduces one of them, namely the branch and bound method.

\section{Branch and bound for mixed-binary optimization}

Branch and bound methods for mixed-binary optimization make use of binary search trees to represent the possible combinations of the binary variables in a structured way. The key idea is to reduce the computational effort by implicit enumeration of the possible combinations of binary variables. In order to do this, branch and bound methods maintain an upper bound on the globally optimal objective function value and compute local lower bounds on the optimal objective function value valid for certain parts of the tree. Often, these bounds can be used to conclude that a certain subtree cannot contain the optimal solution to the original problem and hence can be pruned. Furthermore, these bounds also implies that the methods can be terminated at any time with a certificate that proves the level of suboptimality of the best point found so far. The general concept of the branch and bound method that is presented in this section is based on [23] and [72].

Let $P$ denote the mixed-binary optimization problem to be solved, and let $p_{\mathrm{ub}}$ be an upper bound on the globally optimal objective function value of this problem. The branch and bound method initializes the binary search tree, illustrated in Figure 2.2, with a single node that is called the root node, that holds the original problem $P$. A convex relaxation of $P$, denoted $P_{\mathrm{r}}$, where all binary 
constraints are replaced by interval constraints, is then solved. If the solution to $P_{\mathrm{r}}$ satisfies the binary constraints of $P$, an optimal solution to $P$ has been found and the algorithm terminates. Otherwise, the optimal value of $P_{\mathrm{r}}$, denoted $p_{\mathrm{r}}^{\star}$, is a lower bound on the optimal value of $P$. An upper bound $p_{\mathrm{ub}}$ can be obtained by rounding each of the relaxed binary variables in the solution of $P_{\mathrm{r}}$ and computing the objective function value at this point, provided that it is feasible in $P$.

Branching is then performed by splitting $P$ into two subproblems $P_{0}$ and $P_{1}$. The value of one of the binary variables is fixed to zero in one of the subproblems, and to one in the other. These new subproblems are placed in new nodes, $N_{0}$ and $N_{1}$. Convex relaxations of both these subproblems, where the remaining binary constraints are replaced by interval constraints, are then solved. The optimal value of a convex relaxation of $P_{i}$, denoted $p_{i, \mathrm{r}}^{\star}$, is a lower bound on the objective function value that is valid for the subtree rooted at the corresponding node, $N_{i}$. Thus, if $p_{i, \mathrm{r}}^{\star}>p_{\mathrm{ub}}$, the subtree rooted at $N_{i}$ cannot contain a globally optimal solution and does not need to be further explored. If possible, the solutions to the convex relaxation of the subproblems are also used to tighten the upper bound on the globally optimal objective function value. If rounding the binary variables in the solution of $P_{i, \mathrm{r}}$ yields a point that is feasible in $P$ and gives an objective function value $\bar{p}_{i, \mathrm{r}}^{\star}$ that is smaller than the current upper bound, i.e., $\bar{p}_{i, \mathrm{r}}^{\star}<p_{\mathrm{ub}}$, the upper bound is updated.

By recursively applying this branching technique, the feasible set of the original problem is systematically partitioned until all possible combinations have been explored or pruned and the solution obtained. The conditions for when a subtree rooted at the node $N_{i}$ holding the subproblem $P_{i}$ can be pruned are the following:

- Infeasibility. If the relaxed problem $P_{i, \mathrm{r}}$ is infeasible, then so is $P_{i}$. The subtree rooted at $N_{i}$ will thus not contain any feasible points, and does not need to be further explored.

- Dominance. If the optimal value of $P_{i, \mathrm{r}}$ is greater than an upper bound on the globally optimal value of the original problem $P$, i.e., $p_{i, \mathrm{r}}^{\star}>p_{\mathrm{ub}}$, the subtree rooted at $N_{i}$ cannot contain an optimal solution to $P$ and does not need to be further explored.

- Optimality. If an optimal solution to $P_{i, \mathrm{r}}$ is feasible in $P_{i}$, then it must also be an optimal solution of $P_{i}$. No better solution can be found in the subtree rooted at $N_{i}$, which thus does not need the be further explored.

A general branch and bound method for solving mixed-binary problems that makes use of these pruning criteria is outlined in Algorithm 2.5.

As mentioned earlier, branch and bound methods can be used to generate solutions with a certified level of suboptimality. A $\sigma$-suboptimal solution is defined as follows:

Definition 2.3 ( $\sigma$-suboptimal solution). A $\sigma$-suboptimal solution is a solution to an optimization problem with a corresponding objective function value $\hat{p}$ that satisfies $\hat{p}-p^{\star} \leq \sigma$ if $p^{\star}<\infty$. 
Both absolute suboptimality and relative suboptimality can be considered [5]. In Algorithm 2.5, the absolute suboptimality is controlled by the constant parameter $\epsilon \geq 0$ and the relative suboptimality of the solution is controlled by the constant parameter $\xi \geq 0$. Given a feasible mixed-binary problem $P$ with optimal objective function value $p^{\star}$, the branch and bound method in Algorithm 2.5 terminates with a solution $\hat{x}$ that is an $\left(\epsilon+\xi p^{\star}\right)$-suboptimal solution to $P$, as shown in [5]. If a globally optimal solution is needed, the suboptimality parameters are set to zero. In Example 2.1, the branch and bound method is applied to a small binary linear program.

\section{Algorithm 2.5: Branch and bound}

Input: Mixed-binary problem $P$, absolute suboptimality tolerance $\epsilon$, relative suboptimality tolerance $\xi$

$$
\begin{aligned}
& \text { Initialize: } \\
& p_{\mathrm{ub}}=\infty \\
& p_{\mathrm{lb}}=-\infty \\
& x_{\mathrm{ub}}=\emptyset \\
& \mathcal{L}=\{P\}
\end{aligned}
$$

\section{Search:}

while $\mathcal{L} \neq \emptyset$ do

Pick a problem $P_{i} \in \mathcal{L}$ and set $\mathcal{L}=\mathcal{L} \backslash P_{i}$

$\left(x_{i, \mathrm{r}}^{\star}, p_{i, \mathrm{r}}^{\star}\right) \leftarrow$ Solve relaxed problem $P_{i, \mathrm{r}}$

$\bar{x}_{i, \mathrm{r}}^{\star} \leftarrow$ Round the relaxed binary variables in $x_{i, \mathrm{r}}^{\star}$

$\bar{p}_{i, \mathrm{r}}^{\star} \leftarrow$ Objective function value at $\bar{x}_{i, \mathrm{r}}^{\star}$

if $\bar{p}_{i, \mathrm{r}}^{\star}<p_{\mathrm{ub}}$ and $\bar{x}_{i, \mathrm{r}}^{\star}$ feasible in $P_{i}$ then

$$
x_{\mathrm{ub}}=\bar{x}_{i, \mathrm{r}}^{\star}
$$

end if

$$
p_{\mathrm{ub}}=\bar{p}_{i, \mathrm{r}}^{\star}
$$

if $P_{i, \mathrm{r}}$ infeasible then

Infeasible: No need to expand this node further.

\section{else if $\epsilon+(1+\xi) p_{i, \mathrm{r}}^{\star}>p_{\mathrm{ub}}$ then}

Suboptimal: No need to expand this node further.

else if $x_{i, \mathrm{r}}^{\star}$ feasible in $P_{i}$ then

Optimal in subtree: No need to expand this node further. else

\section{end if}

Split $P_{i}$ at a binary variable into $P_{i, 0}$ and $P_{i, 1}$

$\mathcal{L}=\mathcal{L} \cup\left\{P_{i, 0}, P_{i, 1}\right\}$

\section{end while}

Output: Solution $\hat{x}=x_{\mathrm{ub}}$, objective function value $\hat{p}=p_{\mathrm{ub}}$ 


\section{Example 2.1: Branch and bound applied to a binary linear program}

In this small example, the branch and bound method in Algorithm 2.5 is used to solve the following binary linear program:

$$
\begin{array}{cl}
\underset{\delta}{\operatorname{minimize}} & \delta_{1}+3 \delta_{2}+2 \delta_{3} \\
\text { subject to } & 5 \delta_{1}+3 \delta_{2}+\delta_{3} \geq 6 \\
& \delta_{i} \in\{0,1\}, \quad i=1,2,3 .
\end{array}
$$

Figure 2.3 illustrates the search tree created by the branch and bound method, which finds the optimal solution in five iterations. The solution and optimal value of the corresponding relaxed problem are indicated at each node. The variable that is fixed, and its value, is showed at each edge.

The branch and bound method starts by putting the original problem (2.31), denoted $P$, in the root node of the tree. A convex relaxation of this problem, $P_{\mathrm{r}}$, where the binary constraints are replaced by interval constraints, is then solved. The solution is $\delta_{\mathrm{r}}^{\star}=\left[1, \frac{1}{3}, 0\right]^{\top}$, which yields a lower bound $p_{\mathrm{r}}^{\star}=2$ on the globally optimal value of $P$. No upper bound is obtained at this stage, since rounding $\delta_{\mathrm{r}}^{\star}$ does not produce a point that is feasible in $P$.

Branching is then performed. Two subproblems of $P$ are created by fixing the variable $\delta_{1}$ to zero in one subproblem, $P_{0}$, and to one in the other subproblem, denoted $P_{1}$. The convex relaxation of $P_{0}$ is infeasible, which means that the corresponding subtree does not need be further explored. If there is a feasible solution to $P, \delta_{1}$ must take the value one. The convex relaxation of $P_{1}$ has the same solution as $P_{\mathrm{r}}$, i.e., $\delta_{1, \mathrm{r}}^{\star}=\left[1, \frac{1}{3}, 0\right]^{\top}$. No new bounds on the globally optimal value are obtained in this iteration.

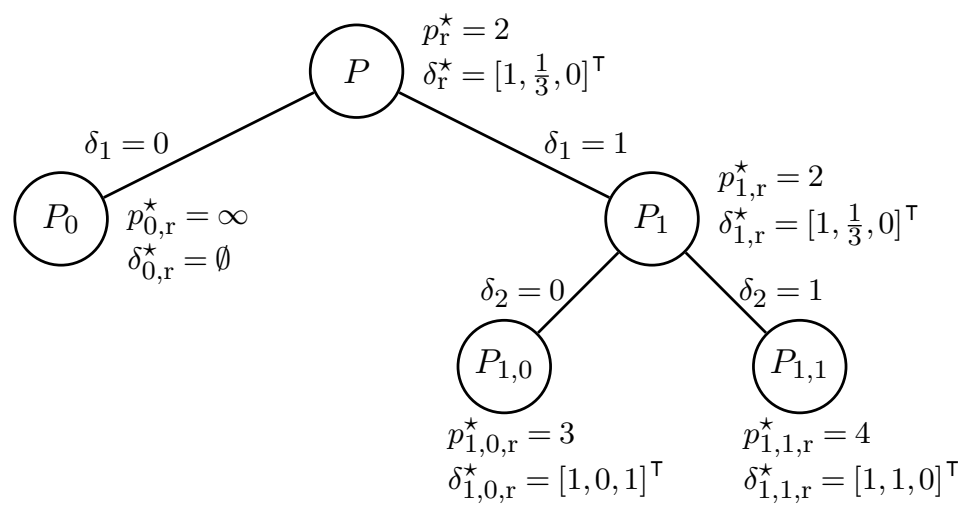

Figure 2.3: Search tree created by branch and bound when solving problem (2.31).

Branching is then performed again. This time by splitting $P_{1}$ at the variable $\delta_{2}$ into $P_{1,0}$ and $P_{1,1}$. Solving the convex relaxation of $P_{1,0}$ yields a solution $\delta_{0,1, \mathrm{r}}^{\star}=$ $[1,0,1]^{\top}$ that is binary feasible in the original problem $P$. This means that an upper bound on the globally optimal value is $p_{\mathrm{ub}}=p_{0,1, \mathrm{r}}^{\star}=3$ and that $\delta_{0,1, \mathrm{r}}^{\star}$ is 
an optimal point in the corresponding subtree, which does not need to be further explored. The optimal value of the convex relaxation of $P_{1,1}$, denoted $p_{1,1, \mathrm{r}}^{\star}$, is attained at the point $\delta_{1,1, \mathrm{r}}^{\star}=[1,0,1]^{\top}$, which is also binary feasible in the original problem. However, since $p_{1,1, \mathrm{r}}^{\star}=4>p_{\mathrm{ub}}$, it is suboptimal and there is no need to further explore the corresponding subtree.

Since there are no more nodes remaining, the branch and bound method has found the globally optimal solution, $\delta^{\star}=[1,0,1]^{\top}$, with the optimal value $p^{\star}=3$, and terminates.

\subsection{Optimal control}

Optimal control theory combines mathematical optimization and control of dynamical systems. It deals with the problem of computing an optimal control input for a given system such that a performance criterion is minimized. For detailed descriptions of optimal control theory, see e.g. [11, 13]. This section provides an overview of optimal control for discrete-time systems.

\subsubsection{Deterministic optimal control}

Consider a dynamical system characterized by the dynamics

$$
x_{k+1}=f\left(x_{k}, u_{k}\right) \text {, }
$$

where $x_{k}$ is the state of the system and $u_{k}$ is the control input, which is constrained to take values in the set $\mathcal{U}_{k}=\mathcal{U}\left(x_{k}\right)$ of admissible control inputs that might depend on the current state $x_{k}$. The set of feasible states at time $k$ is denoted $\mathcal{X}_{k}$. The initial state of the system is assumed to be known and given by $x_{0}=\bar{x}_{0}$.

The objective function that captures the performance criterion of an optimal control problem generally consists of two terms: a terminal cost $\ell_{N}\left(x_{N}\right)$ that penalizes deviation from a desired terminal state, and an accumulation of stage costs $\ell_{k}\left(x_{k}, u_{k}\right)$, which are the costs associated with the state and control input at the time indices $k=1, \ldots, N-1$, where $N$ is the planning horizon.

Given the model of a dynamics, constraints on the states and the control inputs, and an objective function, a discrete-time optimal control problem can be formulated as the following optimization problem:

$$
\begin{array}{cl}
\underset{u_{0: N-1}}{\operatorname{minimize}} & \ell_{N}\left(x_{N}\right)+\sum_{k=0}^{N-1} \ell_{k}\left(x_{k}, u_{k}\right) \\
\text { subject to } & x_{k+1}=f\left(x_{k}, u_{k}\right) \\
& x_{k} \in \mathcal{X}_{k}, \quad u_{k} \in \mathcal{U}_{k} \\
& x_{0}=\bar{x}_{0} .
\end{array}
$$

Solving this optimal control problem gives a sequence of admissible control inputs, which yields a state trajectory that minimizes the objective function. 
Deterministic optimal control problems are formulated in the open-loop sense since the future states can be exactly predicted given an initial state and a sequence of control inputs. Once $u_{k}$ is chosen, $x_{k+1}$ is known, and there is no advantage in waiting until the next time step to decide on $u_{k+1}$.

\subsubsection{Stochastic optimal control}

The dynamical system in (2.32) is deterministic; when a control input is applied at a given state, the next state is fully determined. Problem (2.33) is thus a deterministic optimal control problem. If the dynamical system is instead stochastic, and the succeeding state cannot be exactly determined from a given state and control input due to the presence of disturbances, the optimal control problem becomes a stochastic optimal control problem [11]. The dynamics of such a system can be described by the conditional distribution

$$
x_{k+1} \mid x_{k}, u_{k} \sim p\left(x_{k+1} \mid x_{k}, u_{k}\right),
$$

with prior $x_{0} \sim p\left(x_{0}\right)$, or using the functional representation

$$
x_{k+1}=f\left(x_{k}, u_{k}, w_{k}\right),
$$

where, as in (2.32), $x_{k}$ is the state and $u_{k} \in \mathcal{U}_{k}$ is the control input. The disturbances are modeled as process noise $w_{k}$ characterized by a probability distribution $p\left(w_{k}\right)$. Furthermore, it is assumed that instead of having perfect knowledge of the state, we have access to measurements distributed as

$$
y_{k} \mid x_{k} \sim p\left(y_{k} \mid x_{k}\right)
$$

with the functional representation

$$
y_{k}=h\left(x_{k}, e_{k}\right),
$$

where $e_{k}$ is measurement noise characterized by a given probability distribution.

In stochastic optimal control, the optimization is typically done in the closedloop sense; before the controller commits to applying the next control input to the system, it is allowed to obtain an measurement of the current state. This means that rather than optimizing over control sequences, the optimization is done over control policies [11]. A control policy is a sequence of functions,

$$
\pi=\left\{\mu_{0}, \ldots, \mu_{N-1}\right\}
$$

where $\mu_{k}$ maps the information available to the controller at time $k$, denoted $\tau_{k}$, to a control input $u_{k}=\mu_{k}\left(\tau_{k}\right) \in \mathcal{U}_{k}$. The available information at time $k$ is summarized by the sequence of measurements until time $k$ and applied control inputs until time $k-1$ :

$$
\begin{aligned}
\tau_{0} & =\left\{y_{0}\right\}, \\
\tau_{k+1} & =\left\{\tau_{k}, u_{k}, y_{k+1}\right\}, \quad k=0, \ldots, N-1 .
\end{aligned}
$$


The optimal policy thus provides the optimal control input for every possible information vector $\tau_{k}$, i.e., every possible sequence of measurements and control inputs.

As the dimension of the information vector $\tau_{k}$ increases with the time $k$, there is a need to find a more compact representation of the available information. This can be done using the concept of sufficient statistics [11]. A function $\zeta_{k}$ is referred to as a sufficient statistic if it summarizes all the information in $\tau_{k}$ that is essential for computing the optimal control, i.e.,

$$
u_{k}=\mu_{k}\left(\tau_{k}\right)=\mu_{k}\left(\zeta_{k}\left(\tau_{k}\right)\right) \text {. }
$$

For Markovian systems, a sufficient statistic is the conditional probability distribution $p\left(x_{k} \mid \tau_{k}\right)$ of the state $x_{k}$ given the information vector $\tau_{k}$ [11]. The optimal policy is in this case a function of that probability distribution, i.e., $u_{k}=\mu_{k}\left(p\left(x_{k} \mid \tau_{k}\right)\right)$. Obtaining such a policy naturally requires a substantial amount of computations, but the optimization problem corresponding to finding the optimal policy for Markovian systems can nevertheless be formulated as follows:

$$
\begin{array}{cl}
\underset{\pi}{\operatorname{minimize}} & \mathbf{E}\left\{\ell_{N}\left(x_{N}\right)+\sum_{k=0}^{N-1} \ell_{k}\left(x_{k}, u_{k}\right)\right\} \\
\text { subject to } & x_{k+1}=f\left(x_{k}, u_{k}, w_{k}\right) \\
& y_{k}=h\left(x_{k}, e_{k}\right) \\
& u_{k}=\mu_{k}\left(p\left(x_{k} \mid \tau_{k}\right)\right) \in \mathcal{U}_{k} .
\end{array}
$$

Note that the problem is formulated with the expected cost in the objective function. Due to the uncertainties, the cost is a random variable. A reasonable choice is thus to minimize the expected value of this variable [11]. The expectation is taken with respect to the joint distribution of all the involved random variables, i.e.,

$$
p\left(x_{0: N}, y_{0: N-1}\right)=p\left(x_{0}\right) \prod_{k=0}^{N-1} p\left(x_{k+1} \mid x_{k}, u_{k}\right) p\left(y_{k} \mid x_{k}\right) .
$$

In principle, dynamic programming $[8,11]$ can be applied to compute the optimal policy. The dynamic programming algorithm is based on an intuitive idea called principle of optimality [8], which states the following fact. If the optimal policy for the problem (2.41) is $\pi_{0: N-1}^{\star}=\left\{\mu_{0}^{\star}, \ldots, \mu_{N-1}^{\star}\right\}$, then $\pi_{k: N-1}^{\star}=$ $\left\{\mu_{k}^{\star}, \ldots, \mu_{N-1}^{\star}\right\}$ is the optimal policy for the truncated problem starting at time $k$ and running for the remaining time steps until the planning horizon.

The dynamic programming algorithm starts at time step $N$ by computing

$$
J_{N}^{\star}\left(p\left(x_{N} \mid \tau_{N}\right)\right)=\mathbf{E}\left\{\ell_{N}\left(x_{N}\right) \mid p\left(x_{N} \mid \tau_{N}\right)\right\},
$$

and proceeds backward in time by recursively solving

$$
J_{k}^{\star}\left(p\left(x_{k} \mid \tau_{k}\right)\right)=\min _{u_{k} \in \mathcal{U}_{k}} \mathbf{E}\left\{\ell_{k}\left(x_{k}, u_{k}\right)+J_{k+1}^{\star}\left(p\left(x_{k+1} \mid \tau_{k+1}\right)\right) \mid p\left(x_{k} \mid \tau_{k}\right), u_{k}\right\} .
$$


By letting $\mu_{k}^{\star}\left(p\left(x_{k} \mid \tau_{k}\right)\right)=u_{k}^{\star}$, where $u_{k}^{\star}$ minimizes the right-hand side of $(2.44)$, this recursive algorithm yields the optimal policy $\pi^{\star}=\left\{\mu_{0}^{\star}, \ldots, \mu_{N-1}^{\star}\right\}[11]$.

In theory, dynamic programming gives the optimal solution to stochastic control problems. However, due to the curse of dimensionality [8], computing the optimal policy in closed form is intractable except in special cases, and approximations are necessary. Two popular approximations are described next.

\section{Certainty-equivalent control}

One approximate method is the certainty-equivalent control (CEC) strategy. At each time step $k$, the certainty-equivalent controller applies the control input that would be optimal if all stochastic quantities were replaced by their expected values. The control input $u_{k}=\hat{\mu}_{k}\left(\tau_{k}\right)$ at time $k$ is computed using the following scheme:

1. Compute an estimate of $x_{k}$, i.e., the mean of the conditional distribution of $x_{k}$ given the information available: $\hat{x}_{k}=\mathbf{E} p\left(x_{k} \mid \tau_{k}\right)$.

2. Fix each of the process noise terms at its expected value, i.e., let $\hat{w}_{j}=\mathbf{E} w_{j}$, $j=k, \ldots, N-1$.

3. Compute a sequence of control inputs $\left\{u_{k}, \ldots, u_{N-1}\right\}$ by solving the deterministic optimal control problem:

$$
\begin{array}{cl}
\underset{u_{k: N-1}}{\operatorname{minimize}} & \ell_{N}\left(x_{N}\right)+\sum_{j=k}^{N-1} \ell_{j}\left(x_{j}, u_{j}\right) \\
\text { subject to } & x_{j+1}=f\left(x_{j}, u_{j}, \hat{w}_{j}\right) \\
& x_{k}=\hat{x}_{k}, \quad u_{j} \in \mathcal{U}_{j} .
\end{array}
$$

Note that since the problem is deterministic, the optimization is performed over sequences of control inputs rather than control policies.

4. Apply the first element of the computed sequence of control inputs, $u_{k}$, to the system, and discard the remaining elements. At time $k+1$, the process is repeated from step 1, this time with a one step shorter planning horizon.

The decision variables in the optimization problem solved in each iteration of the certainty-equivalent control loop is a sequence of control inputs to be applied from the current time step $k$ until the end of the planning horizon $N$. As the time $k$ increases toward $N$, the time remaining until the end of the planning horizon decreases and these sequences become shorter.

\section{Receding horizon control}

Another approximate method is the receding horizon control (RHC) strategy, which is also known as model predictive control (MPC) [46]. It resembles the CEC method in the sense that it computes the control input based on the current estimate of the state $x_{k}$ and expected values of future process noise realizations. The difference is that MPC uses the same planning horizon length, denoted $M$, in each iteration 
of the control loop, i.e., the decision variables in the problem solved at time $k$ is a sequence of control inputs from time $k$ until time $k+M-1$. The optimization problem solved at time $k$ in the MPC loop is thus:

$$
\begin{array}{ll}
\underset{u_{k: k+M-1}}{\operatorname{minimize}} & \ell_{k+M}\left(x_{k+M}\right)+\sum_{j=k}^{k+M-1} \ell_{j}\left(x_{j}, u_{j}\right) \\
\text { subject to } & x_{j+1}=f\left(x_{j}, u_{j}, \hat{w}_{j}\right) \\
& x_{k}=\hat{x}_{k}, \quad u_{j} \in \mathcal{U}_{j} .
\end{array}
$$

Since this problem is based on the current state estimate, which is computed using the obtained measurements, the MPC controller operates in closed loop [46].

\subsubsection{Optimal motion planning}

Given a dynamical system, a description of the environment that the system is operating in, an initial state, and a set of goal states, the motion planning problem is to find a sequence of control inputs that drives the system from its initial state to one of the goal states while satisfying the dynamical constraints of the system and avoiding to collide with the surrounding obstacles [36]. In many cases, the focus of a motion planning algorithm is not only to find any feasible solution, but also to compute a feasible solution that is optimal with respect to a performance criterion. The performance criterion is typically encoded in an objective function, and the problem of computing a feasible sequence of control inputs that minimizes an objective function is referred to as optimal motion planning [36]. Commonly used objective functions are e.g. minimizing the energy consumption or the time required to reach the goal. A standard reference on motion planning algorithms is [39] and a survey on methods for self-driving vehicles, which is one of the main applications of motion planning, can be found in [51].

Mathematically, the optimal motion planning problem can be posed as an instance of the optimal control problem:

$$
\begin{array}{cl}
\underset{u_{0: N-1}, N}{\operatorname{minimize}} & \ell_{N}\left(x_{N}\right)+\sum_{k=0}^{N-1} \ell_{k}\left(x_{k}, u_{k}\right) \\
\text { subject to } & x_{k+1}=f\left(x_{k}, u_{k}\right) \\
& x_{k} \in \mathcal{X}_{\text {free }}, \quad u_{k} \in \mathcal{U}_{k} \\
& x_{0}=x_{\text {init }}, \quad x_{N} \in \mathcal{X}_{\text {goal }},
\end{array}
$$

where $x_{\text {init }}$ is the initial state, $\mathcal{X}_{\text {goal }}$ is the set of goal states, and $\mathcal{X}_{\text {free }}$ is the part of the state space that is not occupied by obstacles. The problem is in general nonconvex due to nonlinear system dynamics and complicated descriptions of the obstacles. Solving such problems using numerical optimization is challenging, especially for long time horizons $[10,55]$. Several methods to simplify the search for solutions to motion planning problems have been proposed. Next, we review one of them, namely the lattice-based motion planning approach. 


\section{Lattice-based motion planning}

The key idea in lattice-based motion planning is to convert the numerical optimization problem (2.47) into a graph search problem, without giving up constraints on the system dynamics [56]. This is done by sampling the continuous state space of the system in a regular fashion and constraining its motion to a finite set of motion primitives, that each drives the system from one of the sampled states to another in a dynamically feasible manner. The solution to a motion planning problem can then be obtained by conducting a search in a directed graph $\mathcal{G}=\langle\mathcal{V}, \mathcal{E}\rangle$, referred to as a state lattice [56]. The nodes of the state lattice, $x \in \mathcal{V}$, each represents a state of the system, and the edges $p \in \mathcal{E}$ encode motion primitives that satisfy the system dynamics.

The sampling of the state space defines the states that the system can reach [55]. For a system which state is specified by its two-dimensional position and heading angle, $x=\left[\mathrm{x}_{1} \mathrm{x}_{2} \theta\right]^{\top}$, the sampling implies that $\left(\mathrm{x}_{1}, \mathrm{x}_{2}\right)$ is constrained to values on a grid of resolution $r$, and that $\theta \in \Theta$, where $\Theta$ is a finite set of admissible heading directions. The motion primitives are generated offline using numerical optimal control, which means that constraints on inputs and states can be incorporated to guarantee that the motion primitives are dynamically feasible for the system. A commonly used assumption in the state-lattice motion planning framework is that the system is position-invariant, which means that a motion primitive can be translated and used to connect any two equivalent lattice states [56]. This is illustrated in Figure 2.4. All lattice states are however not equivalent, and to ensure that the computed sequence of motion primitives is dynamically feasible for the system, the motion primitives that can be applied from a given state $x_{k} \in \mathcal{V}$ are constrained to belong to the set of applicable motion primitives from $x_{k}$, denoted $\mathcal{P}\left(x_{k}\right)$. For notational convenience, we introduce the state transition function

$$
x_{k+1}=f_{p}\left(x_{k}, p_{k}\right),
$$

which should be interpreted as starting from the state $x_{k}$, applying the sequence of control inputs that constitutes the motion primitive $p_{k}$, and ending up at the state $x_{k+1}$. Note that the index $k$ here indicates the number of primitives that have been applied since the start, and not time step as in the previous sections, since a motion primitive can last for more than one time step. To ensure that the system avoids the obstacles at all times it is thus not enough to just constrain each $x_{k}$ to belong to $\mathcal{X}_{\text {free }}$. The full trajectory between $x_{k}$ and $x_{k+1}$ when applying $p_{k}$ must avoid collision, which is ensured using the constraint $c\left(x_{k}, p_{k}\right) \in \mathcal{X}_{\text {free }}$.

By discretizing the state space to specify the set of allowed system states $x_{k} \in \mathcal{V}$, and precomputing the set of motion primitives $\mathcal{P}$, the motion planning problem in (2.47) where the optimization is done over the continuum of control inputs $u_{k} \in \mathcal{U}$, 


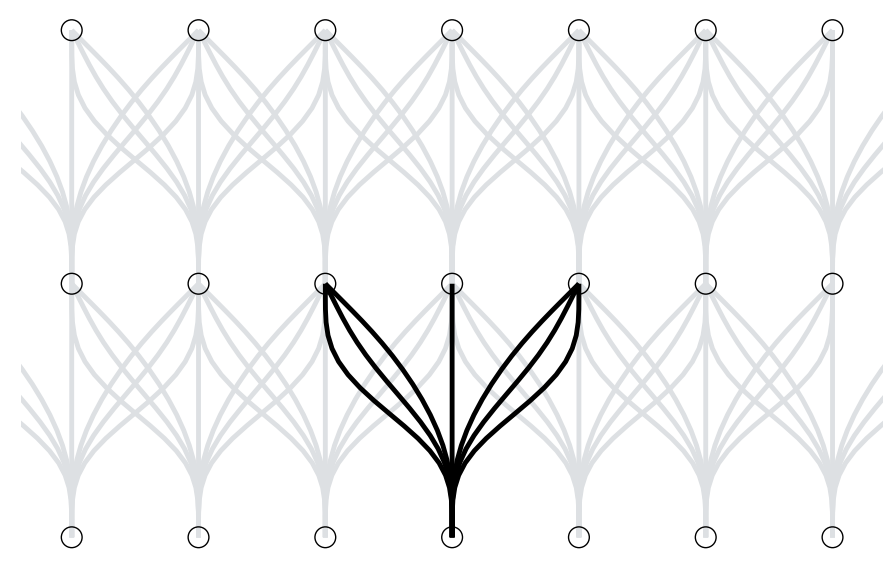

Figure 2.4: Example of equivalent states in a state lattice. The motion primitives (black) are position-invariant and can be applied to all equivalent grid positions of the lattice.

is approximated by the following problem:

$$
\begin{array}{ll}
\underset{p_{0: N-1}, N}{\operatorname{minimize}} & \ell_{N}\left(x_{N}\right)+\sum_{k=0}^{N-1} \ell_{k}\left(x_{k}, p_{k}\right) \\
\text { subject to } & x_{k+1}=f_{p}\left(x_{k}, p_{k}\right) \\
& c\left(x_{k}, p_{k}\right) \in \mathcal{X}_{\text {free }} \\
& p_{k} \in \mathcal{P}\left(x_{k}\right) \\
& x_{0}=x_{\text {init }}, \quad x_{N} \in \mathcal{X}_{\text {goal }},
\end{array}
$$

where the optimization is done over the finite set of motion primitives. This problem can be solved online using efficient graph search methods, e.g., A* [25] or ARA* [43]. The state-lattice approach has been successfully applied in several practical applications, e.g., $[2,44,56]$.

\subsubsection{Informative path planning}

Informative path planning (IPP) [64] considers the problem of optimizing trajectories for mobile sensors in order to maximize the state estimation performance. It can be considered as optimal motion planning with a certain class of performance criteria. In contrast to the typical motion planning problem, there is no set of goal states. Instead, the objective is to compute a trajectory from where the mobile sensor can obtain maximally informative measurements. In IPP, the state estimation problem is thus combined with a stochastic control problem, where decisions are made based on predictions of future measurements. This section formulates a general IPP problem, of which specializations are considered in the following chapters. 
Consider a sensor mounted on a vehicle, a mobile sensor, with state $s_{k}$ at time $k$, that is controlled using the control input $u_{k} \in \mathcal{U}$. The dynamics of the mobile sensor are described by the following model

$$
s_{k+1}=g\left(s_{k}, u_{k}\right) \text {. }
$$

In this thesis, the control input is always connected to the movement of the mobile sensor. However, it could also be used to control other properties of the sensor, e.g., sensing mode or an adjustable beamwidth.

The task of the mobile sensor in an IPP problem is to track the evolution of a system of interest, i.e., gather measurements that are to be used in a state estimation framework. The state of the system of interest, $x_{k}$, is modeled to evolve according to the following dynamics equation:

$$
x_{k+1}=f\left(x_{k}, w_{k}\right)
$$

where $w_{k}$ is process noise characterized by some probability distribution.

The mobile sensor obtains information about the system of interest through imperfect measurements, described by the following measurement model:

$$
y_{k}=h\left(x_{k}, s_{k}, e_{k}\right) \text {, }
$$

where $y_{k}$ is the measurement obtained by the sensor and $e_{k}$ is measurement noise.

Given (2.50)-(2.52), we can formulate a general IPP problem as a stochastic optimal control problem. The information available at time $k$ is summarized by the sensor state trajectory and the obtained measurements:

$$
\begin{aligned}
\tau_{0} & =\left\{s_{0}, y_{0}\right\}, \\
\tau_{k+1} & =\left\{\tau_{k}, s_{k+1}, y_{k+1}\right\}, \quad k=0, \ldots, N-1,
\end{aligned}
$$

for which a sufficient statistic is the current state of the mobile sensor, $s_{k}$, and the conditional probability distribution of the state of the system of interest $p\left(x_{k} \mid \tau_{k}\right)$. The optimal control policy $\pi$ is thus a sequence of functions $\mu_{k}$ that map $s_{k}$ and $p\left(x_{k} \mid \tau_{k}\right)$ to a control input. The optimization problem that corresponds to computing this policy can be formulated as

$$
\begin{array}{ll}
\underset{\pi}{\operatorname{minimize}} & \mathbf{E}\left\{\ell_{N}\left(p\left(x_{N} \mid \tau_{N}\right), s_{N}\right)+\sum_{k=0}^{N-1} \ell_{k}\left(p\left(x_{k} \mid \tau_{k}\right), s_{k}, u_{k}\right)\right\} \\
\text { subject to } & s_{k+1}=g\left(s_{k}, u_{k}\right) \\
& x_{k+1}=f\left(x_{k}, w_{k}\right) \\
& y_{k}=h\left(x_{k}, s_{k}, e_{k}\right) \\
& u_{k}=\mu_{k}\left(p\left(x_{k} \mid \tau_{k}\right), s_{k}\right) \in \mathcal{U}_{k} \\
& s_{0}=\bar{s}_{0},
\end{array}
$$

where $\bar{s}_{0}$ is the initial state of the mobile sensor and the expectation is taken with respect to the joint distribution of all the involved random variables. The goal of 
IPP is to optimize the overall state estimation performance. Hence, the objective function includes a measure of this performance and possibly also costs related to effort of controlling the mobile sensor.

Throughout this thesis, it is assumed that the dynamics of the mobile sensor are deterministic and that its state is perfectly known at all times. Incorporating the notion of uncertainty in the state of the sensor leads to a related problem, often referred to as belief space planning, which has been widely studied [52, 53, 58, 69]. For sensors that do not possess internal states, i.e., $g\left(s_{k}, u_{k}\right)=u_{k}$, the IPP problem coincides with the sensor selection problem. Instead of planning an informative path for a mobile sensor, the sensors are static and the challenge is to select when to activate which sensor. This problem has been studied extensively and several efficient approaches have been proposed $[32,70]$.

Computing an optimal policy by solving the stochastic control problem (2.54) would require a substantial computational effort, and approximations are needed in practice. Neglecting the uncertainty in the dynamics of the state of the system of interest and approximating the conditional distribution of its state by a Gaussian distribution leads to a deterministic problem [57], that can be solved using local numerical optimization [53], sampling-based techniques [28, 38] or graph search methods [4, 63]. 



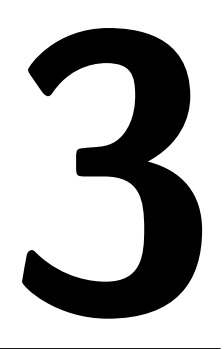

\section{Informative path planning in linear Gaussian settings}

This chapter addresses a special case of the general IPP problem, where the dynamics of the system of interest and the measurements are linear and Gaussian for a given sensor state. Under these assumptions, and for certain classes of objective functions that measure the informativeness of a sensor trajectory, the corresponding stochastic IPP problem reduces to a deterministic optimal control problem [41]. The proof of this is outlined in Section 3.1. In Section 3.2, it is shown that some linear Gaussian IPP problems can be formulated as SDPs. Section 3.3 makes use of the SDP formulation of IPP in a surveillance scenario, and Section 3.4 extends this scenario to also include adversarial observers.

\subsection{Separation principle}

This section shows that a version of the principle of separation of estimation and control [76] holds for linear Gaussian IPP problems. This is a well-known result that was used on a similar problem already in the 1960's [49], but also more recently in $[4,41,63]$. The discussion in this section follows the same chain of arguments as is used in [30], where it is shown that solving an open-loop formulation of the linear Gaussian sensor scheduling problem yields a control sequence that is optimal also in the closed-loop sense.

Let $s_{k}$ denote the state of the sensor and $x_{k}$ the state of a system of interest. Consider a modified version of the linear Gaussian state-space model (2.4):

$$
\begin{aligned}
x_{k+1} & =F x_{k}+G w_{k}, & & w_{k} \sim \mathcal{N}(0, Q), \\
y_{k} & =H\left(s_{k}\right) x_{k}+e_{k}\left(s_{k}\right), & & e_{k}\left(s_{k}\right) \sim \mathcal{N}\left(0, R\left(s_{k}\right)\right) .
\end{aligned}
$$

In contrast to (2.4), the measurement matrix, $H=H\left(s_{k}\right)$, and the covariance matrix of the measurement noise, $R=R\left(s_{k}\right)$, in the above model are not constant, 
but functions of the state of the sensor. The measurement model might depend nonlinearly on the sensor state, but it is important to note that the model is still linear in the state of the system of interest, $x_{k}$. The dynamics of the mobile sensor are deterministic and given by $s_{k+1}=g\left(s_{k}, u_{k}\right)$, where $u_{k}$ is the control input.

The problem of planning the trajectory for a mobile sensor that is estimating the state of the linear Gaussian system in (3.1) can be formulated as an instance of the stochastic IPP problem (2.54):

$$
\begin{array}{ll}
\underset{\pi}{\operatorname{minimize}} & \mathbf{E}\left\{\ell_{N}\left(p\left(x_{N} \mid \tau_{N}\right), s_{N}\right)+\sum_{k=0}^{N-1} \ell_{k}\left(p\left(x_{k} \mid \tau_{k}\right), s_{k}, u_{k}\right)\right\} \\
\text { subject to } & s_{k+1}=g\left(s_{k}, u_{k}\right) \\
& x_{k+1}=F x_{k}+G w_{k} \\
& y_{k}=H\left(s_{k}\right) x_{k}+e_{k}\left(s_{k}\right) \\
& u_{k}=\mu_{k}\left(p\left(x_{k} \mid \tau_{k}\right), s_{k}\right) \in \mathcal{U}_{k} \\
& s_{0}=\bar{s}_{0},
\end{array}
$$

where $\bar{s}_{0}$ is the initial state of the mobile sensor, and $\tau_{k}$ is the information available for decision making at time $k$. The optimization is performed over the space of control policies $\pi=\left\{\mu_{0}, \ldots, \mu_{N-1}\right\}$, and the expectation is taken with respect to the joint distribution of all involved random variables, i.e.,

$$
p\left(x_{0: N}, y_{0: N}\right)=p\left(x_{0}\right) \prod_{k=0}^{N-1} p\left(x_{k+1} \mid x_{k}\right) \prod_{k=0}^{N} p\left(y_{k} \mid x_{k}, s_{k}\right) .
$$

The fact that (3.1) constitutes a linear Gaussian state-space model for the system of interest means that a Kalman filter can be applied to estimate its corresponding state. If the prior distribution of the initial state of the system, $p\left(x_{0}\right)$, is Gaussian, the conditional probability distribution of $x_{k}$ given all measurements up until time $k$ will also be Gaussian according to

$$
p\left(x_{k} \mid y_{1: k}\right)=\mathcal{N}\left(x_{k} \mid \hat{x}_{k \mid k}, P_{k \mid k}\right),
$$

where $\hat{x}_{k \mid k}$ and $P_{k \mid k}$ are computed recursively using the Kalman filter equations from Algorithm 2.1. An equivalent alternative is to use an information filter, in which case the resulting information matrix $\mathcal{I}_{k \mid k}$ is computed using the information matrix recursion

$$
\begin{aligned}
\mathcal{I}_{k \mid k} & =\mathcal{I}_{k \mid k-1}+J_{k}\left(s_{k}\right), \\
\mathcal{I}_{k+1 \mid k} & =\left(F \mathcal{I}_{k \mid k}^{-1} F^{\top}+G Q G^{\top}\right)^{-1},
\end{aligned}
$$

where $J_{k}\left(s_{k}\right)=H^{\top}\left(s_{k}\right) R^{-1}\left(s_{k}\right) H\left(s_{k}\right)$ is the information contained in the measurement obtained at time step $k$. For a more compact notation, we introduce the function $\mathcal{I}_{k+1 \mid k+1}=f_{\mathcal{I}}\left(\mathcal{I}_{k \mid k}, s_{k+1}\right)$, that captures the two update steps of (3.5). As pointed out in Section 2.1.4, a key property of the information matrix recursion is 
that it is invariant to the measurements values, which means that the information matrix of future time steps can be computed in advance.

As discussed in Section 2.1.5, it is reasonable to use an objective function based on the resulting information matrix in order to judge the informativeness of a sensor trajectory. This gives the following terminal and stage cost functions:

$$
\begin{aligned}
\ell_{N}\left(p\left(x_{N} \mid \tau_{N}\right), s_{N}\right) & =\ell_{N}\left(\mathcal{I}_{N \mid N}, s_{N}\right), \\
\ell_{k}\left(p\left(x_{k} \mid \tau_{k}\right), s_{k}, u_{k}\right) & =\ell_{k}\left(\mathcal{I}_{k \mid k}, s_{k}, u_{k}\right), \quad k=0, \ldots, N-1 .
\end{aligned}
$$

The objective function is then independent of all involved random variables, since the state of the mobile sensor $s_{k}$ and control input $u_{k}$ are deterministic quantities, and the information matrix recursion is independent of the actual measurements. This means that the objective function value is deterministic for a given sensor trajectory, i.e., an initial state $\bar{s}_{0}$ and a sequence of control inputs $u_{k}, k=0, \ldots, N-1$. Taking the expectation with respect to all involved random variables thus has no effect.

In general, as described in Section 2.3.4, a sufficient statistic for a stochastic IPP problem is given by the state of the mobile sensor and the conditional distribution of the state of the system of interest. For Gaussian distributions, this is equivalent to the state of the mobile sensor, the current estimate of state of the system of interest, and the corresponding covariance or information matrix. However, the choice of terminal and stage cost functions in (3.6) gives an objective function where the state estimate does not appear explicitly. Since the state estimate also does not affect the information matrix recursion, its value at time $k$ is not needed to find the optimal policy. A sufficient statistic is in this case given by the sensor state $s_{k}$ and the information matrix $\mathcal{I}_{k \mid k}$ corresponding to the state estimate of the system of interest, i.e., $u_{k}=\mu_{k}\left(\mathcal{I}_{k \mid k}, s_{k}\right)$.

The choice of terminal and stage cost functions in (3.6) gives an objective function that is independent of the involved stochastic quantities. It can thus be computed exactly given the initial state of the mobile sensor $\bar{s}_{0}$, the initial information matrix $\overline{\mathcal{I}}_{0 \mid 0}=\left(\operatorname{cov} x_{0}\right)^{-1}$, and the sequence of control inputs $u_{k}, k=0, \ldots, N-1$. This means that the stochastic problem reduces to a deterministic one:

$$
\begin{array}{cl}
\underset{\pi}{\operatorname{minimize}} & \ell_{N}\left(\mathcal{I}_{N \mid N}, s_{N}\right)+\sum_{k=0}^{N-1} \ell_{k}\left(\mathcal{I}_{k \mid k}, s_{k}, u_{k}\right) \\
\text { subject to } & s_{k+1}=g\left(s_{k}, u_{k}\right) \\
& \mathcal{I}_{k+1 \mid k+1}=f_{\mathcal{I}}\left(\mathcal{I}_{k \mid k}, s_{k+1}\right) \\
& u_{k}=\mu_{k}\left(\mathcal{I}_{k \mid k}, s_{k}\right) \in \mathcal{U}_{k} \\
& s_{0}=\bar{s}_{0}, \quad \mathcal{I}_{0 \mid 0}=\overline{\mathcal{I}}_{0 \mid 0} .
\end{array}
$$

An important property of deterministic optimal control problems is that, in contrast to stochastic optimal control problems, the use of feedback does not result in a possible reduction of the optimal objective function value. This means that optimizing over control policies $\pi=\left\{\mu_{0}, \ldots, \mu_{N-1}\right\}$ results in the same optimal value as optimizing over sequences of control inputs $\left\{u_{0}, \ldots, u_{N-1}\right\}[11]$. Hence, 
the linear Gaussian IPP problem can be formulated as the following deterministic problem,

$$
\begin{array}{cl}
\underset{u_{0: N-1}}{\operatorname{minimize}} & \ell_{N}\left(\mathcal{I}_{N \mid N}, s_{N}\right)+\sum_{k=0}^{N-1} \ell_{k}\left(\mathcal{I}_{k \mid k}, s_{k}, u_{k}\right) \\
\text { subject to } & s_{k+1}=g\left(s_{k}, u_{k}\right) \\
& \mathcal{I}_{k+1 \mid k+1}=f_{\mathcal{I}}\left(\mathcal{I}_{k \mid k}, s_{k+1}\right) \\
& s_{0}=\bar{s}_{0}, \quad \mathcal{I}_{0 \mid 0}=\overline{\mathcal{I}}_{0 \mid 0}, \quad u_{k} \in \mathcal{U}_{k},
\end{array}
$$

where the optimization is performed over sequences of control inputs. Even though this problem is deterministic, it is still difficult to solve, since it is both nonlinear and nonconvex. One option would be to try to solve it using one of the many available software packages for nonlinear optimization, e.g., IPOPT [73], ACADO [29] or WORHP [20], but due to the nonconvexity there is no guarantee that a globally optimal solution will be found [18]. Instead, we will focus on a special case of the problem where the relation between the sensor state and the measurement equation makes it possible to reformulate the problem as a convex optimization problem.

\subsection{Semidefinite program formulation}

All equality constraints need to be affine for an optimization problem to be convex. This in not the case for the linear Gaussian IPP problem (3.8), since the equality constraints corresponding to the information matrix recursion are nonlinear, which means that the problem is nonconvex. In this section, it will be shown that under reasonable assumptions, an equivalent convex problem can be obtained by relaxing these nonlinear equality constraints to inequality constraints, which can be further reformulated as linear matrix inequalities.

\subsubsection{Reformulation of nonlinear equality constraints}

For certain classes of nonconvex optimization problems that involve nonlinear equality constraints, it is possible to find the optimal solution by solving convex relaxations of the original problem. Specifically, this is the case when any optimal solution to the relaxed problem satisfies the inequality constraints with equality. This concept is formalized in Theorem 3.1, for which the following definition of matrix monotone (increasing, decreasing) functions will be needed:

Definition 3.1 (Matrix monotone functions, extension of the definition in [18]). Assume $\mathcal{C} \subseteq \mathbf{S}^{n}$. A function $f: \mathbf{S}^{n} \rightarrow \mathbf{S}^{m}$ is called matrix increasing on $\mathcal{C}$ if

$$
X \preceq Y, X \neq Y \Longrightarrow f(X) \preceq f(Y), f(X) \neq f(Y),
$$

for all $X, Y \in \mathcal{C}$. For $m=1, f$ is called matrix increasing on $\mathcal{C}$ if

$$
X \preceq Y, X \neq Y \Longrightarrow f(X)<f(Y) .
$$

Matrix decreasing is defined analogously. 
Some examples of matrix monotone functions of the variable $X \in \mathbf{S}^{n}$ are:

- $\operatorname{tr}(X)$ is matrix increasing [18].

- $\operatorname{tr}\left(X^{-1}\right)$ is matrix decreasing on $\mathbf{S}_{++}^{n}[18]$.

- $\operatorname{det}(X)$ is matrix increasing on $\mathbf{S}_{++}^{n}[18]$.

- The covariance matrix recursion $f_{P}$ is matrix increasing on $\mathbf{S}_{++}^{n}[26]$.

- The information matrix recursion $f_{\mathcal{I}}$ is matrix increasing $\mathbf{S}_{++}^{n}[26]$.

Having defined the notion of matrix monotone functions, the following theorem can be stated.

Theorem 3.1. Assume that

- $\ell_{k}^{X}: \mathbf{S}^{n} \rightarrow \mathbf{R}, k=0, \ldots, N$, are matrix decreasing on $\mathbf{S}_{++}^{n}$,

- $f_{k}(X, y): \mathbf{S}^{n} \times \mathbf{R}^{m} \rightarrow \mathbf{S}^{n}, k=1, \ldots, N$, are finite-valued and matrix increasing in $X$ on $\mathbf{S}_{++}^{n}$ for any feasible $y$,

- $\bar{X}_{0} \in \mathbf{S}_{++}^{n}$.

Then, the optimal solution to the problem

$$
\begin{array}{ll}
\underset{X_{0: N}, y}{\operatorname{minimize}} & \sum_{k=0}^{N} \ell_{k}^{X}\left(X_{k}\right)+f_{0}^{y}(y) \\
\text { subject to } & g(y)=0 \\
& X_{k+1}=f_{k+1}\left(X_{k}, y\right), \quad k=0, \ldots, N-1 \\
& X_{0}=\bar{X}_{0}
\end{array}
$$

coincides with the optimal solution to the problem

$$
\begin{array}{ll}
\underset{X_{0: N}, y}{\operatorname{minimize}} & \sum_{k=0}^{N} \ell_{k}^{X}\left(X_{k}\right)+f_{0}^{y}(y) \\
\text { subject to } & g(y)=0 \\
& X_{k+1} \preceq f_{k+1}\left(X_{k}, y\right), \quad k=0, \ldots, N-1 \\
& X_{0} \preceq \bar{X}_{0} .
\end{array}
$$

Proof: Note that a problem equivalent to (3.10) can be obtained by introducing positive semidefinite slack variables, $\Delta_{k}, k=0, \ldots, N$, and replacing each inequality constraint in (3.10) with an equality constraint. This yields the following 
equivalent problem:

$$
\begin{array}{cll}
\underset{X_{0: N}, \Delta_{0: N}, y}{\operatorname{minimize}} & \sum_{k=0}^{N} \ell_{k}^{X}\left(X_{k}\right)+f_{0}^{y}(y) & \\
\text { subject to } & g(y)=0 & \\
& X_{k+1}+\Delta_{k+1}=f_{k+1}\left(X_{k}, y\right), & \\
& X_{0}+\Delta_{0}=\bar{X}_{0} & \\
& \Delta_{k} \succeq 0, \ldots, N-1
\end{array}
$$

By optimizing over the variables $X_{k}, \Delta_{k}, k=0, \ldots, N$, in (3.11), it will now be shown that the optimal choice of the slack variables is $\Delta_{k}=0, k=0, \ldots, N$, which means that the inequality constraints in (3.10) are satisfied with equality at the optimal solution. We begin by optimizing over the variables in the final stage, namely $X_{N}$ and $\Delta_{N}$. To do this, we introduce the function $f_{0}: \mathbf{S}^{n} \rightarrow \mathbf{R}$, which we assume is matrix decreasing, and make use of the following helper problem

$$
\begin{array}{cl}
\underset{X, \Delta}{\operatorname{minimize}} & f_{0}(X) \\
\text { subject to } & X+\Delta=c_{0} \\
& \Delta \succeq 0,
\end{array}
$$

which can be considered as a parametric program of the parameter $c_{0}$. Since the objective function is matrix decreasing, a nonzero slack $\Delta$ implies a suboptimal $X$ :

$$
X \preceq X+\Delta, X \neq X+\Delta \Longrightarrow f_{0}(X)>f_{0}(X+\Delta) .
$$

Hence, for any finite choice of $c_{0}$, the unique optimal solution to (3.12) is given by $X^{\star}=c_{0}, \Delta^{\star}=0$. In particular, this holds for $c_{0}=f_{k+1}\left(X_{k}, y\right)$, which means that the variables $\Delta_{N}=0, X_{N}=f_{N}\left(X_{N-1}, y\right)$ in (3.11) can be eliminated together with the corresponding equality constraint. This elimination reduces the problem to

$$
\begin{array}{cll}
\underset{X_{0: N-1}, \Delta_{0: N-1}, y}{\operatorname{minimize}} & \sum_{k=0}^{N-1} \ell_{k}^{X}\left(X_{k}\right)+\ell_{N}^{X}\left(f_{N}\left(X_{N-1}, y\right)\right)+f_{0}^{y}(y) \\
\text { subject to } & g(y)=0 & \\
& X_{k+1}+\Delta_{k+1}=f_{k+1}\left(X_{k}, y\right), & \\
& X_{0}+\Delta_{0}=\bar{X}_{0} & \\
& \Delta_{k} \succeq 0, \ldots, N-2
\end{array}
$$

which has the same structure as the original problem (3.11); the objective function is still matrix decreasing in $X_{k}, k=0, \ldots, N-1$, as the second term of the objective function is a matrix decreasing function of a matrix increasing function, the constraint functions are matrix increasing in $X_{k}$ and finite-valued, and $\bar{X}_{0} \in \mathbf{S}_{++}^{n}$.

The procedure of optimizing over, and eliminating, the variables in the final stage can thus be applied repeatedly until all variables except $X_{0}, \Delta_{0}$, and $y$ have 
been eliminated, which eventually gives the equivalent problem

$$
\begin{array}{ll}
\underset{X_{0}, \Delta_{0}, y}{\operatorname{minimize}} & \sum_{k=1}^{N} \ell_{k}^{X}\left(f_{k}\left(\ldots\left(f_{1}\left(X_{0}, y\right), \ldots\right), y\right)\right)+\ell_{0}^{X}\left(X_{0}\right)+f_{0}^{y}(y) \\
\text { subject to } & g(y)=0 \\
& X_{0}+\Delta_{0}=\bar{X}_{0} \\
& \Delta_{0} \succeq 0 .
\end{array}
$$

Since also this problem has the same structure as (3.11), the optimal and finite choice of $X_{0}$ and $\Delta_{0}$ is given by $X_{0}=\bar{X}_{0}, \Delta_{0}=0$. This means that for any feasible $y$, the optimal choice of $X_{k}$ and $\Delta_{k}$ in (3.11) and thus also in (3.10) is given by $X_{0}^{\star}=\bar{X}_{0}, X_{k+1}^{\star}=f_{k+1}\left(X_{k}^{\star}, y\right)$ for $k=0, \ldots, N-1$, and $\Delta_{k}^{\star}=0$ for $k=0, \ldots, N$, and that $X_{k}^{\star}$, by induction, is finite for all $k$. The optimal choice of $y$ can be obtained by solving

$$
\begin{array}{ll}
\underset{y}{\operatorname{minimize}} & \sum_{k=1}^{N} \ell_{k}^{X}\left(f_{k}\left(\ldots\left(f_{1}\left(\bar{X}_{0}, y\right), \ldots\right), y\right)\right)+\ell_{0}^{X}\left(\bar{X}_{0}\right)+f_{0}^{y}(y) \\
\text { subject to } & g(y)=0 .
\end{array}
$$

Note that the constraints of (3.10) are relaxations of the constraints of (3.9). Hence, the feasible set of (3.9) is a subset of the feasible set of (3.10) and the optimal value of (3.9) can thus not be lower than the optimal value of (3.10). Since $X_{0}^{\star}=\bar{X}_{0}, X_{k+1}^{\star}=f_{k}\left(X_{k}^{\star}, y\right), k=0, \ldots, N-1$, is optimal in (3.10) and feasible in (3.9), it must also be optimal in (3.9). Hence, the optimal solution to (3.10) coincides with the optimal solution to (3.9), which concludes the proof.

\subsubsection{Application to informative path planning}

The information matrix recursion $f_{\mathcal{I}}$ is matrix increasing [26] and finite-valued provided that the largest eigenvalue of $J_{k}\left(s_{k}\right)$ is finite. Assume that the following conditions are satisfied for (3.8):

- The objective function is separable in $\mathcal{I}_{k \mid k}$ and $\left(s_{k}, u_{k}\right)$, i.e.,

$$
\begin{aligned}
\ell_{N}\left(\mathcal{I}_{N \mid N}, s_{N}\right) & =\ell_{N}^{\mathcal{I}}\left(\mathcal{I}_{N \mid N}\right)+\ell_{N}^{s}\left(s_{N}\right), \\
\ell_{k}\left(\mathcal{I}_{k \mid k}, s_{k}, u_{k}\right) & =\ell_{k}^{\mathcal{I}}\left(\mathcal{I}_{k \mid k}\right)+\ell_{k}^{s, u}\left(s_{k}, u_{k}\right), \quad k=0, \ldots, N-1,
\end{aligned}
$$

- The stage cost functions $\ell_{k}^{\mathcal{I}}, k=0, \ldots, N$, are matrix decreasing on $\mathbf{S}_{++}^{n}$,

- The initial information matrix $\overline{\mathcal{I}}_{0 \mid 0}$ is positive definite.

Then, Theorem 3.1 can be applied to the problem (3.8), and the equality constraints representing the information matrix recursion can equivalently be replaced by inequality constraints. These new inequality constraints can be further reformulated. Assuming that the matrices $F$ and $Q$ are positive definite, which implies 
that their inverses exist and are positive definite, the Woodbury matrix identity (2.12) gives

$$
\begin{aligned}
& \mathcal{I}_{k+1 \mid k+1} \preceq f_{\mathcal{I}}\left(\mathcal{I}_{k \mid k}, s_{k+1}\right)=\left(F \mathcal{I}_{k \mid k}^{-1} F^{\top}+G Q G^{\top}\right)^{-1}+J_{k+1}\left(s_{k+1}\right) \Longleftrightarrow \\
& \mathcal{I}_{k+1 \mid k+1} \preceq F^{-\top} \mathcal{I}_{k \mid k} F^{-1}+J_{k+1}\left(s_{k+1}\right)-F^{-\top} \mathcal{I}_{k \mid k} F^{-1} G \times \\
& \quad \times\left(Q^{-1}+G^{\top} F^{-\top} \mathcal{I}_{k \mid k} F^{-1} G\right)^{-1} G^{\top} F^{-\top} \mathcal{I}_{k \mid k} F^{-1} F^{\top} Q^{-1} .
\end{aligned}
$$

Furthermore, since the assumption of positive definite $Q$ and $\mathcal{I}_{k \mid k}$ implies that

$$
Q^{-1}+G^{\top} F^{-\top} \mathcal{I}_{k \mid k} F^{-1} G \succ 0
$$

the Schur complement condition for positive semidefiniteness of a block matrix [18] can be applied to (3.18), which gives:

$$
\begin{aligned}
& 0 \preceq\left[\begin{array}{cc}
F^{-\mathrm{\top}} \mathcal{I}_{k \mid k} F^{-1}-\mathcal{I}_{k+1 \mid k+1}+J_{k+1}\left(s_{k+1}\right) & F^{-\mathrm{\top}} \mathcal{I}_{k} F^{-1} G \\
G^{\mathrm{\top}} F^{-\mathrm{\top}} \mathcal{I}_{k} F^{-1} & Q^{-1}+G^{\mathrm{\top}} F^{-\mathrm{\top}} \mathcal{I}_{k} F^{-1} G
\end{array}\right] \\
& \triangleq \Gamma\left(\mathcal{I}_{k \mid k}, \mathcal{I}_{k+1 \mid k+1}, J_{k+1}\left(s_{k+1}\right)\right) .
\end{aligned}
$$

This eliminates the use of the inverse of the information matrix in the constraint. Instead, (3.20) is a linear matrix inequality in $\mathcal{I}_{k \mid k}, \mathcal{I}_{k+1 \mid k+1}$, and $J_{k+1}\left(s_{k+1}\right)$. Hence, under the above listed assumptions, the problem (3.8) can be equivalently reformulated as

$$
\begin{array}{cl}
\underset{z}{\operatorname{minimize}} & \ell_{N}\left(\mathcal{I}_{N \mid N}, s_{N}\right)+\sum_{k=0}^{N-1} \ell_{k}\left(\mathcal{I}_{k \mid k}, s_{k}, u_{k}\right) \\
\text { subject to } & s_{k+1}=g\left(s_{k}, u_{k}\right) \\
& 0 \preceq \Gamma\left(\mathcal{I}_{k \mid k}, \mathcal{I}_{k+1 \mid k+1}, J_{k+1}\left(s_{k+1}\right)\right) \\
& s_{0}=\bar{s}_{0}, \quad \mathcal{I}_{0 \mid 0}=\overline{\mathcal{I}}_{0 \mid 0},
\end{array}
$$

where $z=\left\{\mathcal{I}_{k \mid k}, k=0, \ldots, N, u_{0: N-1}\right\}$. If the objective function is convex, the function $g$ that describes the sensor dynamics is linear, and the information contained in a measurement, $J_{k}$, is an affine function of the sensor state, this is a convex problem.

\section{Example 3.1: Position-dependent measurement quality}

Consider the IPP problem of planning the trajectory of a mobile sensor with the task of estimating the state of a system of interest before going back to the base at the origin. Assume that the measurement noise level depends on the position of the mobile sensor, which has to move to a different part of the area to gather information about the system of interest.

The dynamics of the mobile sensor are modeled as a discretized near-constant velocity model, where the state $s=\left[\begin{array}{llll}\mathrm{s}_{1} & \mathrm{~s}_{2} & \mathrm{v}_{1} & \mathrm{v}_{2}\end{array}\right]^{\top}$ represents the sensor's twodimensional position and velocity, and the control input $u_{k}$ corresponds to its 
acceleration:

$$
s_{k+1}=\underbrace{\left[\begin{array}{cc}
I & \tau I \\
0 & I
\end{array}\right]}_{\triangleq A} s_{k}+\underbrace{\left[\begin{array}{c}
\frac{\tau^{2}}{2} I \\
\tau I
\end{array}\right]}_{\triangleq B} u_{k},
$$

where $\tau$ is the sampling period. The acceleration $u_{k}$ is limited to have a magnitude of less than one in each direction. The state of the system of interest evolves according to a random walk model driven by white Gaussian noise with unit covariance:

$$
x_{k+1}=x_{k}+w_{k}, \quad w_{k} \sim \mathcal{N}(0, I) .
$$

The measurement model is linear in the state of the system of interest and the measurement noise is white and Gaussian, and the covariance matrix is a function of the sensor state:

$$
\begin{aligned}
y_{k} & =x_{k}+e_{k}\left(s_{k}\right), \quad e_{k}\left(s_{k}\right) \sim \mathcal{N}\left(0, R\left(s_{k}\right)\right), \\
R^{-1}\left(s_{k}\right) & =\left(100+\mathrm{s}_{1, k}\right) I .
\end{aligned}
$$

The relation between sensor state and measurement noise is selected for illustrational purposes rather than describing a real-world sensor. An information filter, where the information matrix recursion depends on the state of the mobile sensor, is used to estimate the state of the system of interest:

$$
\mathcal{I}_{k+1 \mid k+1}=f_{\mathcal{I}}\left(\mathcal{I}_{k \mid k}, s_{k+1}\right)
$$

With the initial state of the mobile sensor given by $\bar{s}_{0}=[8,0,0,0]^{\top}$, the initial information matrix $\mathcal{I}_{0 \mid 0}=I$, a planning horizon of $N=15$ time steps, and terminal and stage cost functions given by

$$
\begin{aligned}
\ell_{N}\left(\mathcal{I}_{N \mid N}, s_{N}\right) & =-\operatorname{tr}\left(\mathcal{I}_{N \mid N}\right)+100\left\|s_{N}\right\|_{2}^{2}, \\
\ell_{k}\left(\mathcal{I}_{k \mid k}, s_{k}, u_{k}\right) & =-\operatorname{tr}\left(\mathcal{I}_{k \mid k}\right), \quad k=0, \ldots, N-1,
\end{aligned}
$$

the corresponding linear Gaussian IPP problem can be formulated as:

$$
\begin{array}{cl}
\underset{u_{0: N-1}}{\operatorname{minimize}} & 100\left\|s_{N}\right\|_{2}^{2}-\sum_{k=0}^{N} \operatorname{tr}\left(\mathcal{I}_{k \mid k}\right) \\
\text { subject to } & s_{k+1}=A s_{k}+B u_{k} \\
& -1 \preceq u_{k} \preceq 1 \\
& \mathcal{I}_{k+1 \mid k+1}=f_{\mathcal{I}}\left(\mathcal{I}_{k \mid k}, s_{k+1}\right) \\
& s_{0}=\bar{s}_{0}, \quad \mathcal{I}_{0 \mid 0}=I .
\end{array}
$$

This problem satisfies all requirements of Theorem 3.1: $-\operatorname{tr}\left(\mathcal{I}_{k \mid k}\right)$ is matrix decreasing [18], $f_{\mathcal{I}}$ is matrix increasing [26], and $I \in \mathbf{S}_{++}^{n}$. The constraints $\mathcal{I}_{k+1 \mid k+1}=$ $f_{\mathcal{I}}\left(\mathcal{I}_{k \mid k}, s_{k+1}\right)$ can thus be replaced by $\mathcal{I}_{k+1 \mid k+1} \preceq f_{\mathcal{I}}\left(\mathcal{I}_{k \mid k}, s_{k+1}\right)$, which can be further reformulated as $0 \preceq \Gamma\left(\mathcal{I}_{k \mid k}, \mathcal{I}_{k+1 \mid k+1}, J\left(s_{k+1}\right)\right)$, where $\Gamma$ is defined in $(3.20)$ 


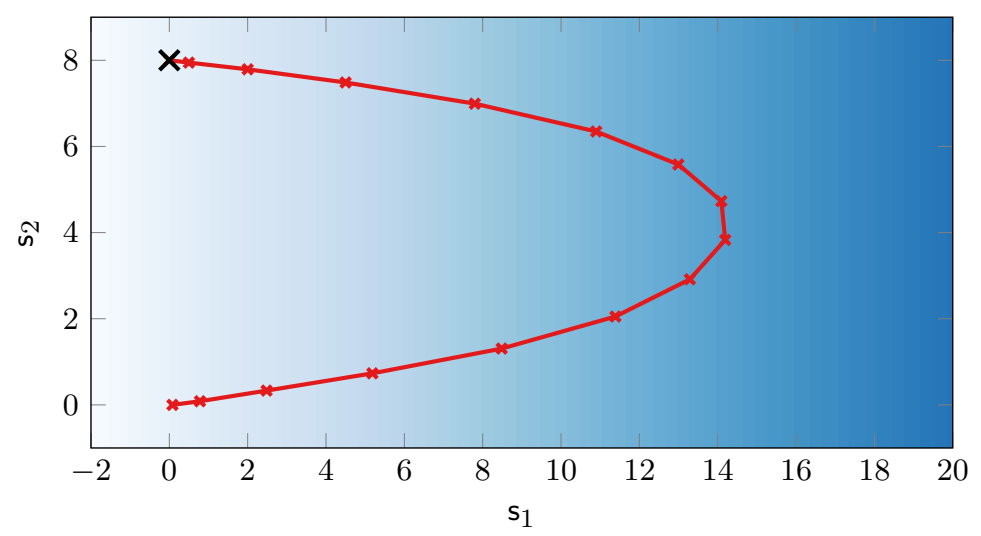

Figure 3.1: The mobile sensor starts at $(0,8)$ and the goal is to collect as much information as possible and go to the origin in fifteen time steps. The amount of information contained in a measurement from a point in space is color-dependent: white is less informative, blue is more informative.

and $J\left(s_{k}\right)=\left(100+s_{1, k}\right) I$. Hence, $(3.27)$ is equivalent to the following convex problem:

$$
\begin{array}{ll}
\underset{z}{\operatorname{minimize}} & 100\left\|s_{N}\right\|_{2}^{2}-\sum_{k=0}^{N} \operatorname{tr}\left(\mathcal{I}_{k \mid k}\right) \\
\text { subject to } & s_{k+1}=A s_{k}+B u_{k} \\
& -1 \preceq u_{k} \preceq 1 \\
& 0 \preceq \Gamma\left(\mathcal{I}_{k \mid k}, \mathcal{I}_{k+1 \mid k+1},\left(100+\mathrm{s}_{1, k+1}\right) I\right) \\
& s_{0}=\bar{s}_{0}, \quad \mathcal{I}_{0 \mid 0}=I,
\end{array}
$$

where $z=\left\{\mathcal{I}_{k \mid k}, k=0, \ldots, N, u_{0: N-1}\right\}$. The optimal solution to this problem, obtained using MOSEK [3], gives the sensor trajectory illustrated in Figure 3.1.

\subsection{Informative path planning for surveillance}

In this section, we consider an application of IPP where a mobile sensor with limited sensing range is used to monitor the evolution of a number of features in a given environment. The features are separated in space, which means that the sensor is unable to obtain measurements of all features at the same time, and the problem is to compute a trajectory for the mobile sensor such that it is able to keep track of the state of all features. With the assumptions that the dynamics of the features are linear and that the measurements equation is linear in the state of the features, the corresponding IPP problem can be formulated as a deterministic optimal control problem. The considered scenario is illustrated in Figure 3.2. 


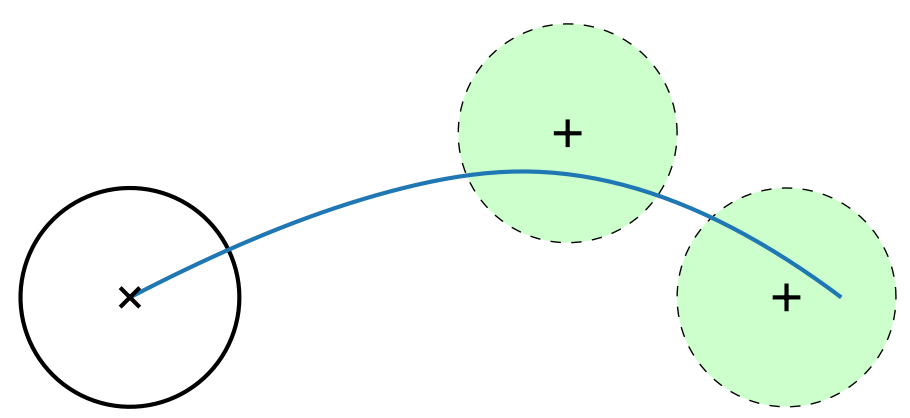

Figure 3.2: A mobile robot equipped with a sensor can obtain noisy measurements of static features in the environment if they are within the sensor's sensing range. The problem, which is illustrated in the figure, is to find a sensing path that is maximizing the gathered information. The initial position of the mobile sensor is indicated by the $\mathrm{x}$, and the black circle illustrates its sensing range. A feature (marked with + ) is visible to the mobile sensor if the sensor is located within the corresponding green region.

\subsubsection{Problem formulation}

This section defines the sensor and dynamics models, and formulates a deterministic IPP problem.

\section{Motion and measurement models}

Consider a mobile sensor with deterministic and linear dynamics, given by

$$
s_{k+1}=A s_{k}+B u_{k},
$$

where $s_{k}$ is the state of the mobile sensor and $u_{k}$ is the control input, both at time $k$. The set of feasible states is denoted by $\mathcal{S}$ and the set of admissible control inputs is denoted by $\mathcal{U}$. The sets $\mathcal{S}$ and $\mathcal{U}$ are both assumed to be convex sets.

The mobile sensor is operating in an environment where there are a number of distinct features at known locations. The superindex $(i)$ will be used for quantities related to the $i$ th feature. The position of the $i$ th feature, denoted $p_{k}^{(i)}$, is assumed to be known at all times and is not considered to be part of the state of the feature, which instead corresponds to an associated property of interest, representing e.g. air quality or temperature at the feature's position. The state of a feature evolves according to a linear model

$$
x_{k+1}^{(i)}=F^{(i)} x_{k}^{(i)}+G^{(i)} w_{k}^{(i)}, \quad w_{k}^{(i)} \sim \mathcal{N}\left(0, Q^{(i)}\right),
$$

where $x_{k}^{(i)}$ is the state of the feature, and the process noise $w_{k}^{(i)}$ is white and Gaussian with covariance $Q^{(i)} \succ 0$. The states of different features are assumed 
to be mutually independent and the state transition matrix $F^{(i)}$ is assumed to be non-singular for each feature. The total number of features in the environment is denoted $L$.

The mobile sensor has a limited sensing range and obtains measurements of features that are in the vicinity of its current position. The set of points from which measurements can be obtained, referred to as the sensing region, is thus a function of the state of the mobile sensor and is defined as $\mathcal{V}\left(s_{k}\right)=\left\{p \mid f_{\mathcal{V}}\left(s_{k}, p\right) \leq 0\right\}$. Assuming that $f_{\mathcal{V}}$ is a convex function, $\mathcal{V}\left(s_{k}\right)$ is a convex set. The measurement model is discontinuous in the sense that a linear measurement of the state of the feature is obtained if the feature is within the sensing region at time $k$, and no measurement otherwise. As argued in [65], not obtaining a measurement is equivalent to obtaining a measurement with an infinite noise covariance. The measurement equation can thus be written

$$
y_{k}^{(i)}=H^{(i)} x_{k}^{(i)}+e_{k}^{(i)}\left(s_{k}, p_{k}^{(i)}\right), \quad e_{k}^{(i)}\left(s_{k}, p_{k}^{(i)}\right) \sim \mathcal{N}\left(0, R^{(i)}\left(s_{k}, p_{k}^{(i)}\right)\right),
$$

where the measurement noise is white and Gaussian with covariance

$$
R^{(i)}\left(s_{k}, p_{k}^{(i)}\right)= \begin{cases}\bar{R}^{(i)}, & p_{k}^{(i)} \in \mathcal{V}\left(s_{k}\right) \\ \sigma^{2} I, & p_{k}^{(i)} \notin \mathcal{V}\left(s_{k}\right)\end{cases}
$$

where $\sigma^{2} \rightarrow \infty$.

\section{Information representation}

The measurement model (3.31) is linear in the state of the feature, $x_{k}^{(i)}$, and nonlinear only in the state of the sensor, $s_{k}$. Together with the dynamics model (3.30), it constitutes a linear Gaussian state-space model for the state of the feature. Hence, a linear information filter can be employed to estimate its state from the obtained measurements. As described in Section 2.1.4, the information filter maintains an information matrix to represent the accuracy of the state estimate. The recursion for the information matrix corresponding to the $i$ th feature becomes

$$
\begin{aligned}
\mathcal{I}_{k+1 \mid k+1}^{(i)} & =f_{\mathcal{I}}\left(\mathcal{I}_{k \mid k}^{(i)}, s_{k+1}, p_{k+1}^{(i)}\right) \\
& =\left(F^{(i)} \mathcal{I}_{k \mid k}^{(i)^{-1}} F^{(i)^{\top}}+G^{(i)} Q^{(i)} G^{(i)^{\top}}\right)^{-1}+J^{(i)}\left(s_{k+1}, p_{k+1}^{(i)}\right),
\end{aligned}
$$

where

$$
\begin{aligned}
& J^{(i)}\left(s_{k}, p_{k}^{(i)}\right)=H^{(i)^{\top}}\left(R^{(i)}\left(s_{k}, p_{k}^{(i)}\right)\right)^{-1} H^{(i)} \\
& = \begin{cases}H^{(i)^{\top}} \bar{R}^{(i)^{-1}} H^{(i)}, & p_{k}^{(i)} \in \mathcal{V}\left(s_{k}\right) \\
0, & p_{k}^{(i)} \notin \mathcal{V}\left(s_{k}\right)\end{cases}
\end{aligned}
$$

is the information contained in the measurement of the $i$ th feature at time $k$. For notational convenience further on, we introduce the constant matrix

$$
\bar{J}^{(i)}=H^{(i)^{\top}} \bar{R}^{(i)^{-1}} H^{(i)} .
$$


Note that the information matrix recursion does not depend on the actual measurement values, but only on whether or not measurements are obtained. Since the position of each feature and the state of the sensor is considered to be perfectly known at each point in time, it is possible to use the information filter to predict the accuracy of future estimates before the actual measurements have been obtained.

\section{Optimization problem}

The task of the mobile sensor is to monitor the evolution of the state of the features. Since the sensor's noise characteristics depend on the state of the mobile sensor, the choice of trajectory through the environment will affect the sensor's monitoring performance. The problem of finding the best trajectory for the mobile sensor can be formulated as an IPP problem, where the performance is captured by a measure of the resulting information matrices in the objective function. The objective function could also contain penalties on the state of the mobile sensor or the control input to e.g. enable a trade-off between informativeness and energy consumption. For the remainder of this section, we will use an objective function composed of the following terminal and stage cost functions:

$$
\begin{aligned}
& \ell_{N}\left(\left\{\mathcal{I}_{N \mid N}^{(i)}\right\}_{i=1}^{L}, s_{N}\right)=s_{N}^{\top} V_{s} s_{N}-\left(\operatorname{det} \mathcal{I}_{N}\right)^{1 / n}, \\
& \ell_{k}\left(\left\{\mathcal{I}_{k \mid k}^{(i)}\right\}_{i=1}^{L}, s_{k}, u_{k}\right)=u_{k}^{\top} V_{u} u_{k}+s_{k}^{\top} V_{s} s_{k}-\left(\operatorname{det} \mathcal{I}_{k}\right)^{1 / n}, k=0, \ldots, N-1,
\end{aligned}
$$

where $V_{u}$ and $V_{s}$ are positive semidefinite weight matrices, $\mathcal{I}_{k}=\operatorname{blkdiag}\left(\left\{\mathcal{I}_{k \mid k}^{(i)}\right\}_{i=1}^{L}\right)$ and $n$ is the number of rows in $\mathcal{I}_{k}$.

The problem of finding the best trajectory for the mobile sensor can thus be formulated as

$$
\begin{array}{cl}
\underset{u_{0: N-1}}{\operatorname{minimize}} & \sum_{k=0}^{N-1} u_{k}^{\top} V_{u} u_{k}+\sum_{k=0}^{N}\left(s_{k}^{\top} V_{s} s_{k}-\left(\operatorname{det} \mathcal{I}_{k}\right)^{1 / n}\right) \\
\text { subject to } & s_{k+1}=A s_{k}+B u_{k} \\
& \mathcal{I}_{k+1 \mid k+1}^{(i)}=f_{\mathcal{I}}\left(\mathcal{I}_{k \mid k}^{(i)}, s_{k+1}, p_{k+1}^{(i)}\right) \\
& u_{k} \in \mathcal{U}, \quad s_{k} \in \mathcal{S} \\
& s_{0}=\bar{s}_{0}, \quad \mathcal{I}_{0 \mid 0}^{(i)}=\overline{\mathcal{I}}_{0 \mid 0}^{(i)},
\end{array}
$$

where $\bar{s}_{0}$ is the initial state of the mobile sensor and $\overline{\mathcal{I}}_{0 \mid 0}^{(i)}, i=1, \ldots, L$, are the initial information matrices corresponding to the estimated states of the features. In addition to involving nonlinear equality constraints, which by definition makes the problem nonconvex, the problem is nonsmooth due to the discontinuities in the measurement model. Finding the optimal sequence of control inputs by directly solving the problem formulated as a continuous nonlinear optimization program is thus challenging, since there is no gradient information that can guide the solver towards the regions where measurements can be obtained. Instead, in the next section, we will formulate the problem as a mixed-binary program. 


\subsubsection{Reformulation to mixed-binary semidefinite program}

Assuming that the initial information matrices $\overline{\mathcal{I}}_{0 \mid 0}^{(i)}, i=1, \ldots, L$, are positive definite, the problem (3.37) fulfills the requirements of Theorem 3.1:

- The information part of the stage cost, $\ell_{k}^{\mathcal{I}}=-\left(\operatorname{det} \mathcal{I}_{k}\right)^{1 / n}, k=0, \ldots, N$, is matrix decreasing on $\mathbf{S}_{++}^{n}[18]$.

- The constraint function $f_{\mathcal{I}}$, corresponding to the information matrix recursion, is matrix decreasing for all feasible values of $s_{k}[26]$.

- The constraint function $f_{\mathcal{I}}$ is finite-valued given that the largest eigenvalue of $J^{(i)}\left(s_{k}\right)$ is finite for all $s_{k}$.

The theorem can thus be applied, and the equality constraints representing the information matrix recursion can equivalently be replaced by inequality constraints, which can be further reformulated as the linear matrix inequalities

$$
0 \preceq \Gamma\left(\mathcal{I}_{k \mid k}^{(i)}, \mathcal{I}_{k+1 \mid k+1}^{(i)}, J^{(i)}\left(s_{k+1}, p_{k+1}^{(i)}\right)\right), \quad k=0, \ldots, N-1, i=1, \ldots, L,
$$

where the function $\Gamma$ is defined in (3.20).

The discrete nature of the measurement model can be modeled by introducing an auxiliary binary variable $\delta_{k}^{(i)}$ for each time step $k$ and feature $i$. This variable is a nonlinear function of the state of the sensor, $s_{k}$, and the position of the $i$ th feature, $p_{k}^{(i)}$, and takes the value one if a measurement of the $i$ th feature is obtained at time step $k$, and the value zero otherwise:

$$
\delta_{k}^{(i)}=\delta\left(s_{k}, p_{k}^{(i)}\right)= \begin{cases}1, & p_{k}^{(i)} \in \mathcal{V}\left(s_{k}\right) \\ 0, & p_{k}^{(i)} \notin \mathcal{V}\left(s_{k}\right)\end{cases}
$$

This logic relation between $s_{k}$ and $\delta_{k}^{(i)}$ can be modeled using a big- $M$ formulation [71], as will be described next. The following constraint forces the binary variable $\delta_{k}^{(i)}$ to take the value zero when the $i$ th feature is outside of the mobile sensor's sensing region, i.e., when $f_{\mathcal{V}}\left(s_{k}, p_{k}^{(i)}\right)>0$ :

$$
f_{\mathcal{V}}\left(s_{k}, p_{k}^{(i)}\right)-\left(1-\delta_{k}^{(i)}\right) M_{\delta} \leq 0
$$

where $M_{\delta}$ is a constant coefficient representing a known upper bound for $f_{\mathcal{V}}\left(s_{k}, p_{k}^{(i)}\right)$. Logically, (3.40) achieves the condition

$$
f_{\mathcal{V}}\left(s_{k}, p_{k}^{(i)}\right)>0 \rightarrow \delta_{k}^{(i)}=0
$$

where ' $\rightarrow$ ' stands for 'implies'. This provides a sufficient link between $s_{k}$ and $\delta_{k}^{(i)}$ in this IPP problem, since the condition

$$
f_{\mathcal{V}}\left(s_{k}, p_{k}^{(i)}\right) \leq 0 \rightarrow \delta_{k}^{(i)}=1
$$


is achieved implicitly at optimality. This follows from that although $\delta_{k}^{(i)}=0$ does not violate the constraints if $f_{\mathcal{V}}\left(s_{k}, p_{k}^{(i)}\right) \leq 0$, it is suboptimal since $\delta_{k}^{(i)}=1$ is also feasible and results in a measurement update of the corresponding information matrix, and thereby yields a better objective function value.

With the introduction of the binary variables, the information contained in a measurement, defined in (3.34), can be written $J^{(i)}\left(s_{k}, p_{k}^{(i)}\right)=\delta_{k}^{(i)} \bar{J}^{(i)}$. By letting $z=\left\{\mathcal{I}_{k \mid k}^{(i)}, k=0, \ldots, N, i=1, \ldots, L, \delta_{1: N}^{(i)}, i=1, \ldots, L, u_{0: N-1}\right\}$, the problem $(3.37)$ can thus be equivalently reformulated as

$$
\begin{array}{cl}
\underset{z}{\operatorname{minimize}} & \sum_{k=0}^{N-1} u_{k}^{\top} V_{u} u_{k}+\sum_{k=0}^{N}\left(s_{k}^{\top} V_{s} s_{k}-\left(\operatorname{det} \mathcal{I}_{k}\right)^{1 / n}\right) \\
\text { subject to } & s_{k+1}=A s_{k}+B u_{k} \\
& 0 \preceq \Gamma\left(\mathcal{I}_{k \mid k}^{(i)}, \mathcal{I}_{k+1 \mid k+1}^{(i)}, \delta_{k+1}^{(i)} \bar{J}^{(i)}\right) \\
& f_{\mathcal{V}}\left(s_{k}, p_{k}^{(i)}\right) \leq\left(1-\delta_{k}^{(i)}\right) M_{\delta} \\
& u_{k} \in \mathcal{U}, \quad s_{k} \in \mathcal{S}, \quad \delta_{k}^{(i)} \in\{0,1\} \\
& s_{0}=\bar{s}_{0}, \quad \mathcal{I}_{0 \mid 0}^{(i)}=\overline{\mathcal{I}}_{0 \mid 0}^{(i)},
\end{array}
$$

which is a mixed-binary SDP problem. The relaxed problem that is obtained by relaxing the binary constraints $\delta_{k}^{(i)} \in\{0,1\}$ in (3.43) to interval constraints $\delta_{k}^{(i)} \in[0,1]$ is thus a convex SDP problem. As described in Section 2.2.2, this means that a branch and bound method can be applied to efficiently find the globally optimal solution, or alternatively to solve the problem to any user-defined level of suboptimality. Furthermore, the equivalence between the original problem (3.37) and the reformulated problem (3.43) implies that their optimal solutions coincide, and that the globally optimal solution to (3.37) can be obtained by solving (3.43). Introducing the binary variables directly in the original problem formulation (3.37) would have resulted in nonconvex relaxations that are of little use in branch and bound, since that problem involves nonlinear equality constraints. The key to finding a globally optimal trajectory for the mobile sensor is thus the application of Theorem 3.1 .

\subsubsection{Numerical illustrations}

In this section, the performance of the mixed-binary SDP formulation of the IPP problem is compared to the performance of the rapidly exploring information gathering tree (RIG-tree) algorithm from [28]. The RIG-tree algorithm is a samplingbased method that is based on the rapidly expanding random tree (RRT) algorithm [40] for path planning. The RIG-tree algorithm was implemented in Python while the optimization problems in the proposed approach were solved in Matlab 2017b using YALMIP's implementation of a standard branch and bound algorithm, BnB, [45] which uses MOSEK [3] to solve the convex relaxations. All simulations were 


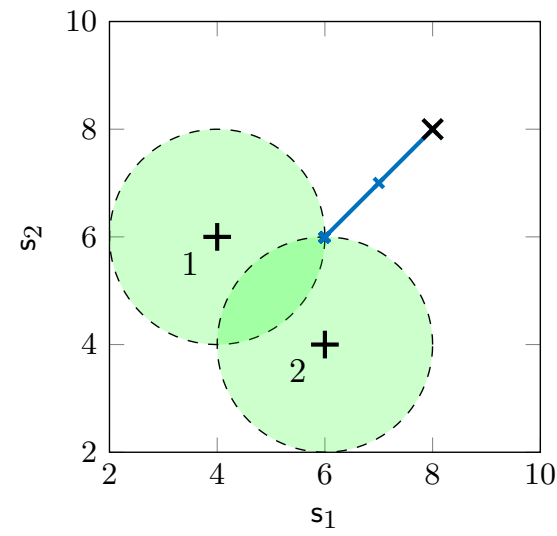

(a) Scenario 1

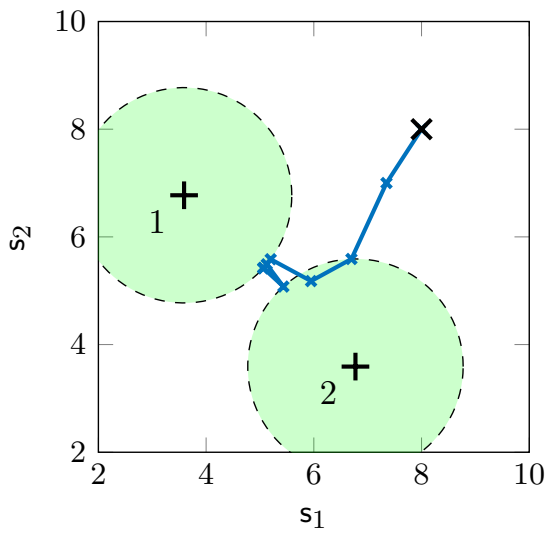

(b) Scenario 2

Figure 3.3: Resulting trajectories for a mobile sensor starting at the point marked with $x$ with the objective of maximizing the acquired information of the two features marked with + . The green regions represent the areas from where the features are visible to the sensor.

run on a laptop with a $2.6 \mathrm{GHz}$ Intel i7 processor with $16 \mathrm{~GB}$ of RAM running Ubuntu 16.04.

The performance evaluation is based on the two scenarios that are illustrated in Figure 3.3. Both scenarios involve two stationary features of interest, whose properties evolve according to random walk models,

$$
x_{k+1}^{(i)}=x_{k}^{(i)}+w_{k}^{(i)}, \quad w_{k}^{(i)} \sim \mathcal{N}\left(0, Q^{(i)}\right),
$$

where the process noise covariance is ten times larger for feature 1 (upper left) than for feature 2: $Q^{(1)}=10 I, Q^{(2)}=I$. The higher noise level implies that a higher measurement rate is required to keep the state estimate of the first feature accurate, compared to the second feature. The state of the mobile sensor represents its two-dimensional position and velocity, $s=\left[\begin{array}{llll}s_{1} & s_{2} & v_{1} & v_{2}\end{array}\right]^{\top}$, and the control input corresponds to its acceleration, which is limited to $u_{k} \in \mathcal{U}=\{u \mid-1 \preceq u \preceq 1\}$. The sensing region is a circle with radius $2 \mathrm{~m}$ centered in the mobile sensor's current position. The measurement that is obtained if the $i$ th feature is within the sensing region at time $k$ is defined by

$$
y_{k}^{(i)}=x_{k}^{(i)}+e_{k}^{(i)}, \quad e_{k}^{(i)} \sim \mathcal{N}(0, I) .
$$

As previously, the measurement noise covariance is considered to be infinite if the feature is outside the sensing region. The planning horizon is $N=10$ time steps and the weight matrices in the objective function are given by

$$
V_{s}=\left[\begin{array}{cc}
0 & 0 \\
0 & 0.01 I
\end{array}\right], \quad V_{u}=0 .
$$



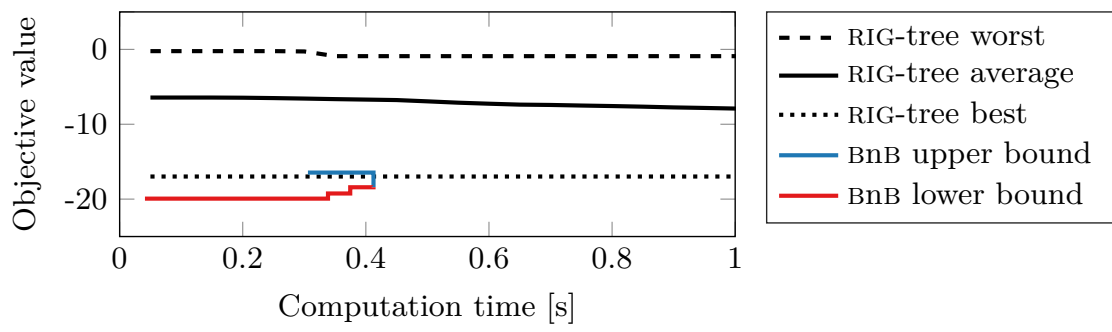

(a) Scenario 1

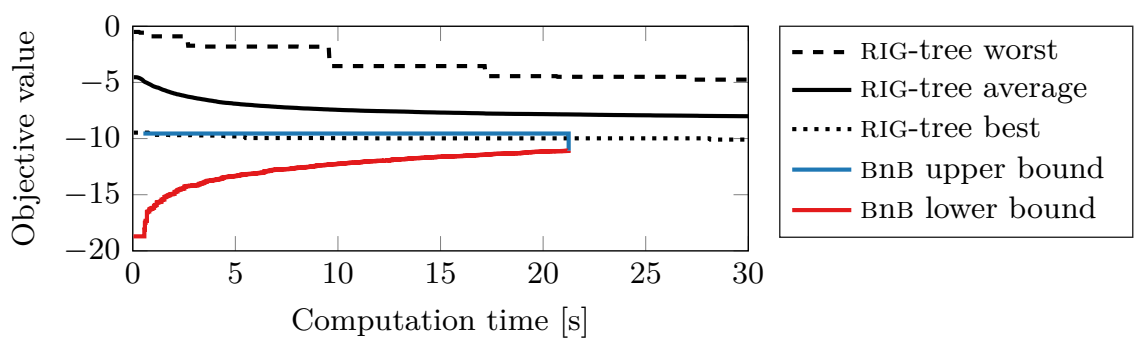

(b) Scenario 2

Figure 3.4: Objective value as a function computation time in seconds. The RIG-tree data is generated using 1000 Monte Carlo runs.

Hence, there is a small penalty on the velocity of the mobile sensor and no penalty on the control input.

Figure 3.3 shows the optimized trajectories obtained using the proposed mixedbinary SDP formulation. In Scenario 1, the sensor moves as fast as possible to a point from where both features are visible, where it remains static. In Scenario 2, there is no such point. Instead, the sensor makes a quick visit to feature 2 and then spends most time near the feature with high process noise covariance.

Figure 3.4 illustrates how the objective function value improves as a function of the computation time for the proposed approach and the RIG-tree algorithm. Since the RIG-tree algorithm is a sampling-based method that returns different results each time it is called, and the performance in terms of objective function value thus varies, Monte Carlo simulations are used to generate the relevant data. YALMIP's BnB quickly finds a feasible solution which is nearly as good as the best solution found by the RIG-tree algorithm in all simulations and significantly better than the average solution. When given more time, BnB finds a better solution than the RIG-tree algorithm and also proves that it is a globally optimal solution. In contrast to the proposed method, the RIG-tree does not provide a certificate of the level of suboptimality, which means that there is no way of knowing how far the obtained solution is from an optimal one. 


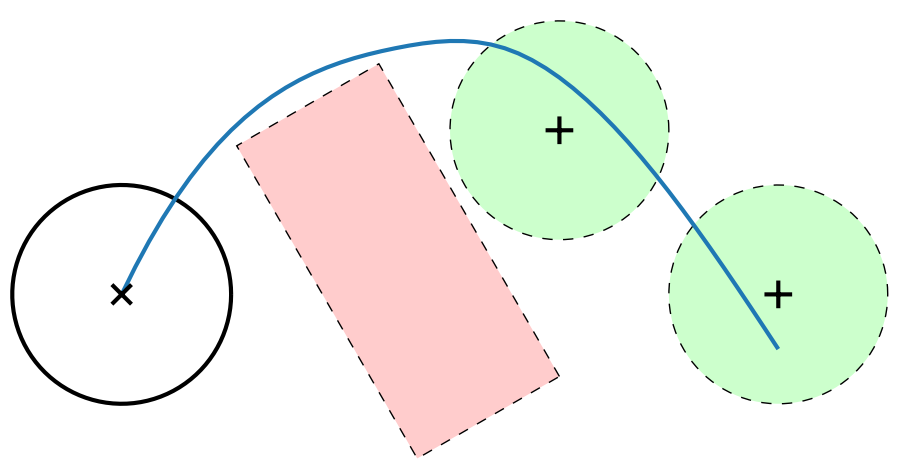

Figure 3.5: The scenario illustrated in Figure 3.2 is here extended with an adversarial observer with limited sensing region (red area) that tracks the mobile sensor. The problem is to find a sensing path that is maximizing the gathered information of the features while minimizing the tracking performance of the adversarial observer.

\subsection{Avoiding adversarial observers}

In this section, we extend the work on global optimization for IPP from the previous section to also handle scenarios that include adversarial observers that should be avoided. Gathering information about features of interest is no longer the only task for the mobile sensor; it also has to avoid being tracked by an opponent while doing so. Figure 3.5 illustrates the considered problem.

The problem of planning paths to minimize the exposure to adversarial observers, i.e., computing stealthy paths, has received some attention in the literature, and a survey of different approaches can be found in [1]. We will make use of binary variables to indicate whether the adversarial observer obtains measurements of the mobile sensor. There is thus a connection to collision avoidance problems, that can be solved using mixed-integer optimization [60], but the adversarial sensing regions in this section are not to be considered as obstacles, as the mobile sensor is allowed to travel through them, at a certain cost.

\subsubsection{Problem formulation}

We begin by considering only the avoidance part of the problem illustrated in Figure 3.5 and define the involved models and the corresponding optimization problem in this section. In Section 3.4.3, the stealthy path planning problem is then combined with the IPP problem.

\section{Dynamics and measurement models}

In the environment that the mobile sensor is operating in, there is now an adversarial observer that seeks to estimate the state of the mobile sensor. The adversarial 
observer is static and obtains measurements of the mobile sensor if it is located within a limited sensing region denoted $\Pi$. The measurement model is defined by

$$
y_{k}= \begin{cases}H_{s} s_{k}+e_{k}, & s_{k} \in \Pi \\ \emptyset, & s_{k} \notin \Pi\end{cases}
$$

where $y_{k}$ and $e_{k}$ are the measurement output and measurement noise, respectively. The measurement noise is assumed to be white and Gaussian with mean zero and covariance $R_{s} \succ 0$.

The discrete nature of the measurement model is captured by a binary variable $\gamma_{k}$ for each time step, that indicates whether the sensor can obtain a measurement or not. The variable $\gamma_{k}=\gamma\left(s_{k}, \Pi\right)$ is a function of the state of the mobile sensor and takes the value one if $s_{k}$ is within the adversarial observer's sensing region $\Pi$ and the value zero otherwise:

$$
\gamma_{k}= \begin{cases}1, & s_{k} \in \Pi \\ 0, & s_{k} \notin \Pi .\end{cases}
$$

\section{Information model}

The adversarial observer is assumed to employ a Kalman filter to estimate the state of the mobile sensor. The true dynamics of the mobile sensor might be unknown to the adversarial, and a general linear motion model is applied to represent its dynamics:

$$
s_{k+1}=F_{s} s_{k}+w_{k}, \quad w_{k} \sim \mathcal{N}\left(0, Q_{s}\right),
$$

where $w_{k}$ is the process noise which is assumed to be white and Gaussian with covariance $Q_{s}$.

Let $P_{k \mid k}$ be the covariance matrix corresponding to the adversarial observer's estimate of $s_{k}$ given all measurements up until and including time step $k$ and let $P_{k}$ denote the one-step predicted covariance matrix:

$$
P_{k}=P_{k \mid k-1}=F_{s} P_{k-1 \mid k-1} F_{s}^{\top}+Q_{s} .
$$

The recursion for the covariance matrix of the filtered estimates can then be written as

$$
\begin{aligned}
P_{k \mid k} & =f_{P}\left(P_{k-1 \mid k-1}, \gamma_{k}\right) \\
& =P_{k}-\gamma_{k} P_{k} H_{s}^{\top}\left(H_{s} P_{k} H_{s}^{\top}+R_{s}\right)^{-1} H_{s} P_{k} .
\end{aligned}
$$

Note that $\gamma_{k}=0$ corresponds to propagating the previous covariance matrix when there is no measurement update available at time $k$.

Since a Kalman filter is used to track the mobile sensor, an indication of the adversarial observer's tracking performance can be obtained by a measure of the resulting covariance matrix $P_{k \mid k}$. The problem here is to minimize the tracking performance, which corresponds to making the covariance matrix as big as possible. 


\section{Nonlinear mixed-binary optimization problem}

The stealthy path planning problem can then be formally stated as the following mixed-binary program:

$$
\begin{array}{cl}
\underset{u_{0: N-1}, \gamma_{1: N}}{\operatorname{minimize}} & \ell_{N}\left(P_{N \mid N}, s_{N}\right)+\sum_{k=0}^{N-1} \ell_{k}\left(P_{k \mid k}, s_{k}, u_{k}\right) \\
\text { subject to } & s_{k+1}=A s_{k}+B u_{k} \\
& P_{k+1 \mid k+1}=f_{P}\left(P_{k \mid k}, \gamma_{k+1}\right) \\
& \gamma_{k}=\gamma\left(s_{k}, \Pi\right) \in\{0,1\} \\
& u_{k} \in \mathcal{U}, \quad s_{k} \in \mathcal{S} \\
& s_{0}=\bar{s}_{0}, \quad P_{0 \mid 0}=\bar{P}_{0 \mid 0},
\end{array}
$$

where $\bar{s}_{0}$ is the initial state of the mobile sensor and $\bar{P}_{0 \mid 0}$ is the covariance matrix corresponding to the adversarial observer's estimate of $s_{0}$. The binary variables are considered as auxiliary decision variables connected to the state of the mobile sensor via constraints. The terminal and stage cost functions are assumed to be separable in $\left(s_{k}, u_{k}\right)$ and $P_{k \mid k}$, i.e.,

$$
\begin{aligned}
\ell_{N}\left(P_{N \mid N}, s_{N}\right) & =w_{s, u} \ell_{N}^{s}\left(s_{N}\right)+w_{P} \ell_{N}^{P}\left(P_{N \mid N}\right), \\
\ell_{k}\left(P_{k \mid k}, s_{k}, u_{k}\right) & =w_{s, u} \ell_{k}^{s, u}\left(s_{k}, u_{k}\right)+w_{P} \ell_{k}^{P}\left(P_{k \mid k}\right), \quad k=0, \ldots, N-1,
\end{aligned}
$$

where $w_{s, u}$ and $w_{P}$, are weighting parameters.

\subsubsection{Computing globally optimal solutions}

Branch and bound methods make use of relaxations of the original problem when searching for the globally optimal solution to a mixed-integer program. The relaxations are obtained by relaxing the binary constraints $\gamma_{k} \in\{0,1\}$, often to interval constraints $\gamma_{k} \in[0,1]$. A prerequisite for the search to be correct is that the relaxations are convex problems, to guarantee that they can be solved to global optimality and hence give correct lower bounds. Since the stealthy path planning problem (3.52) includes nonlinear equality constraints (3.51), a straight-forward continuous relaxation of the problem is a nonconvex problem [18]. In this section, it is shown that the problem can be reformulated as an equivalent problem where the continuous relaxations are convex problems.

\section{Convex reformulation of the nonlinear equality constraints}

Assuming that the initial covariance matrix $\bar{P}_{0 \mid 0}$ is positive definite and that $\ell_{k}^{P}, k=0, \ldots, N$, is matrix decreasing on $\mathbf{S}_{++}^{n}$, the stealthy path planning problem (3.52) fulfills the requirements of Theorem 3.1 since the constraint function $f_{P}$, corresponding to the covariance matrix recursion, is finite valued and matrix decreasing for $\gamma \in\{0,1\}[26]$. The theorem can thus be applied, and the equality 
constraints representing the covariance matrix recursion (3.51) can equivalently be replaced by inequality constraints according to

$$
\begin{aligned}
P_{k \mid k} & \preceq f_{P}\left(P_{k-1 \mid k-1}, \gamma_{k}\right) \\
& =P_{k}-\gamma_{k} P_{k} H_{s}^{\top}\left(H_{s} P_{k} H_{s}^{\top}+R_{s}\right)^{-1} H_{s} P_{k} .
\end{aligned}
$$

Similarly as with the IPP problem in Section 3.3, the new inequality constraints can be further rewritten. By inserting $\gamma_{k}$ at appropriate places, which is possible since $\gamma_{k}$ is binary, and applying the Schur complement condition for positive semidefiniteness of a block matrix [18], the following equation is obtained:

$$
\begin{array}{rlr}
P_{k \mid k} & \preceq P_{k}-\gamma_{k} P_{k} H_{s}^{\top}\left(H_{s} P_{k} H_{s}^{\top}+R_{s}\right)^{-1} H_{s} P_{k} & \Longleftrightarrow \\
P_{k \mid k} \preceq P_{k}-\gamma_{k} P_{k} H_{s}^{\top}\left(\gamma_{k} H_{s} P_{k} H_{s}^{\top}+R_{s}\right)^{-1} H_{s} P_{k} \gamma_{k} & \Longleftrightarrow \\
0 & \preceq\left[\begin{array}{cc}
P_{k}-P_{k \mid k} & \gamma_{k} P_{k} H_{s}^{\top} \\
H_{s} P_{k} \gamma_{k} & \gamma_{k} H_{s} P_{k} H_{s}^{\top}+R_{s}
\end{array}\right], &
\end{array}
$$

where the last inequality involves products of the binary variable $\gamma_{k}$ and the continuous variable $P_{k}$. Following [9], a linear representation of this product can be obtained by introducing an auxiliary real variable $\widetilde{P}_{k} \triangleq \gamma_{k} P_{k}$ that satisfies

$$
\widetilde{P}_{k}= \begin{cases}P_{k}, & \gamma_{k}=1 \\ 0, & \gamma_{k}=0 .\end{cases}
$$

By letting $M_{k}$ be an over-estimate of the largest eigenvalue of $P_{k}$ and $m_{k}$ be an under-estimate of the smallest eigenvalue of $P_{k}$, the relation $\widetilde{P}_{k}=\gamma_{k} P_{k}$ is imposed by the following set of inequalities:

$$
\begin{aligned}
& \widetilde{P}_{k} \preceq \gamma_{k} M_{k} I, \\
& \widetilde{P}_{k} \succeq \gamma_{k} m_{k} I, \\
& \widetilde{P}_{k} \preceq P_{k}-\left(1-\gamma_{k}\right) m_{k} I, \\
& \widetilde{P}_{k} \succeq P_{k}-\left(1-\gamma_{k}\right) M_{k} I .
\end{aligned}
$$

Note that appropriate values for $m_{k}$ and $M_{k}$ can be obtained by propagating the initial covariance matrix $\bar{P}_{0 \mid 0}$ through (3.51) with $\gamma=1$ and $\gamma=0$, respectively.

Inserting the new auxiliary variable $\widetilde{P}_{k}$ into $(3.55)$ gives

$$
0 \preceq\left[\begin{array}{cc}
P_{k}-P_{k \mid k} & \widetilde{P}_{k} H_{s}^{\top} \\
H_{s} \widetilde{P}_{k} & H_{s} \widetilde{P}_{k} H_{s}^{\top}+R_{s}
\end{array}\right],
$$

which is a linear matrix inequality in $P_{k \mid k}, P_{k}$, and $\widetilde{P}_{k}$. An equivalent, linear, representation of the nonlinear constraint (3.55) is thus given by (3.57) and (3.58).

\section{Representation of the adversarial sensing region}

It is assumed that the adversarial sensing region can be described by a polyhedron in the form of the intersection of $q$ halfspaces:

$$
\Pi=\left\{s \mid A_{\Pi} s \preceq b_{\Pi}\right\} .
$$


The logic relation (3.48) between $s_{k}$ and $\gamma_{k}$ can then be modeled using a big- $M$ formulation [71] and an auxiliary binary vector $\tilde{\gamma}$. The following constraints:

$$
\begin{aligned}
& A_{\Pi} s_{k}-b_{\Pi} \preceq\left(1-\tilde{\gamma}_{k}\right) M_{\gamma}, \\
& A_{\Pi} s_{k}-b_{\Pi} \succ-\tilde{\gamma}_{k} M_{\gamma},
\end{aligned}
$$

where $M_{\gamma}$ is a sufficiently large constant coefficient, force all elements of the auxiliary binary vector $\tilde{\gamma}_{k}$ to take the value one when $s_{k} \in \Pi$ and at least one element of $\tilde{\gamma}_{k}$ to take the value zero when $s_{k} \notin \Pi$. This means that the condition $\gamma_{k}=1$ if and only if $s_{k} \in \Pi$ can be achieved by letting the product of all elements in $\tilde{\gamma}_{k}$ represent $\gamma_{k}$, i.e.,

$$
\gamma_{k}=\prod_{i=1}^{q} \tilde{\gamma}_{k}^{i}
$$

where superscript $i$ denotes the $i$ th element of the corresponding vector. The product of binary variables is equivalent to the following set of inequalities [9]:

$$
\begin{aligned}
\gamma_{k}-\tilde{\gamma}_{k}^{i} & \leq 0, \quad i=1, \ldots, q, \\
-\gamma_{k}+\sum_{i=1}^{q} \tilde{\gamma}_{k}^{i} & \leq q-1 .
\end{aligned}
$$

Hence, the auxiliary binary vector $\tilde{\gamma}_{k}$ together with (3.60) and (3.62) can be used to represent the constraint $\gamma_{k}=\gamma\left(s_{k}, \Pi\right)$. Since both (3.60) and (3.62) are linear constraints, they are convex constraints when $\gamma$ and $\tilde{\gamma}$ are considered as continuous variables.

Note that (3.60a), which achieves the condition

$$
s_{k} \in \Pi \rightarrow \gamma_{k}=1
$$

provides a sufficient link between $s_{k}$ and $\gamma_{k}$ in this case. The condition

$$
s_{k} \notin \Pi \rightarrow \gamma_{k}=0
$$

is achieved implicitly at optimality since if both $\gamma_{k}=0$ and $\gamma_{k}=1$ are feasible, $\gamma_{k}=0$ will result in a better objective function value. However, including (3.60b) in the problem formulation might be computationally desirable [71]. 


\section{Reformulated problem}

Using the reformulations described in the previous two subsections, an equivalent formulation of (3.52) is given by the following mixed-binary SDP:

$$
\begin{array}{ll}
\underset{z}{\operatorname{minimize}} & \ell_{N}\left(P_{N \mid N}, s_{N}\right)+\sum_{k=0}^{N-1} \ell_{k}\left(P_{k \mid k}, s_{k}, u_{k}\right) \\
\text { subject to } & s_{k+1}=A s_{k}+B u_{k} \\
& 0 \preceq\left[\begin{array}{cc}
P_{k}-P_{k \mid k} & \widetilde{P}_{k} H_{s}^{\top} \\
H_{s} \widetilde{P}_{k} \quad H_{s} \widetilde{P}_{k} H_{s}^{\top}+R_{s}
\end{array}\right] \\
& \widetilde{P}_{k} \preceq \gamma_{k} M_{k} I \\
& \widetilde{P}_{k} \succeq \gamma_{k} m_{k} I \\
& \widetilde{P}_{k} \preceq P_{k}-\left(1-\gamma_{k}\right) m_{k} I \\
& \widetilde{P}_{k} \succeq P_{k}-\left(1-\gamma_{k}\right) M_{k} I \\
& P_{k}=F_{s} P_{k-1 \mid k-1} F_{s}^{\top}+Q_{s} \\
& A_{\Pi} s_{k}-b_{\Pi} \preceq\left(1-\tilde{\gamma}_{k}\right) M_{\gamma} \\
& A_{\Pi} s_{k}-b_{\Pi} \succ-\tilde{\gamma}_{k} M_{\gamma} \\
& \gamma_{k}-\tilde{\gamma}_{k}^{i} \leq 0 \\
& -\gamma_{k}+\sum_{i=1}^{q} \tilde{\gamma}_{k}^{i} \leq q-1, \\
& s_{k} \in \mathcal{S}, \quad u_{k} \in \mathcal{U}, \quad \gamma_{k}, \tilde{\gamma}_{k}^{i} \in\{0,1\}, \\
& s_{0}=\bar{s}_{0}, \quad P_{0 \mid 0}=\bar{P}_{0 \mid 0} .
\end{array}
$$

where $z=\left\{P_{k \mid k}, k=0, \ldots, N, \widetilde{P}_{1: N}, u_{0: N-1}, \gamma_{1: N}, \tilde{\gamma}_{1: N}\right\}$.

Relaxing the binary constraints $\gamma_{k}, \tilde{\gamma}_{k}^{i} \in\{0,1\}$ in (3.65) to interval constraints $\gamma_{k}, \tilde{\gamma}_{k}^{i} \in[0,1]$ yields a convex SDP problem. This means that a branch and bound method can be used to solve the problem to global optimality, or to a user-defined certified level of suboptimality. Furthermore, the equivalence between the original problem (3.52) and (3.65) implies that the optimal solutions to both problems coincide, and that a globally optimal solution to the seemingly intractable problem (3.52) can be obtained by solving the tractable problem (3.65).

\subsubsection{Stealthy informative path planning}

In this section, the stealthy path planning problem (3.52) is combined with the IPP problem from Section 3.3 and the problem illustrated in Figure 3.5 is formulated as a mixed-binary nonlinear program. For simplicity, we formulate the problem for just one feature of interest located at $p_{k}$, but the results hold also for the case with multiple features. The problem of computing the best trajectory for the mobile 
sensor can be formulated as the following optimization problem:

$$
\begin{array}{cl}
\underset{z}{\operatorname{minimize}} & \ell_{N}\left(\mathcal{I}_{N \mid N}, P_{N \mid N}, s_{N}\right)+\sum_{k=0}^{N-1} \ell_{k}\left(\mathcal{I}_{k \mid k}, P_{k \mid k}, s_{k}, u_{k}\right) \\
\text { subject to } & s_{k+1}=A s_{k}+B u_{k} \\
& P_{k+1 \mid k+1}=f_{P}\left(P_{k \mid k}, \gamma_{k+1}\right) \\
& \gamma_{k}=\gamma\left(s_{k}, \Pi\right) \in\{0,1\} \\
& \mathcal{I}_{k+1 \mid k+1}=f_{\mathcal{I}}\left(\mathcal{I}_{k \mid k}, \delta_{k+1}\right) \\
& \delta_{k}=\delta\left(s_{k}, p_{k}\right) \in\{0,1\} \\
& u_{k} \in \mathcal{U}, \quad s_{k} \in \mathcal{S} \\
& s_{0}=\bar{s}_{0}, \quad P_{0 \mid 0}=\bar{P}_{0 \mid 0}, \quad \mathcal{I}_{0 \mid 0}=\overline{\mathcal{I}}_{0 \mid 0},
\end{array}
$$

where $z=\left\{u_{0: N-1}, \gamma_{1: N}, \delta_{1: N}\right\}$ is a concatenation of the decision variables, $\bar{s}_{0}$ is the initial state of the mobile sensor, $\bar{P}_{0 \mid 0}$ is the covariance matrix corresponding to the adversarial observer's estimate of $s_{0}$, and $\overline{\mathcal{I}}_{0 \mid 0}$ is the initial information matrix corresponding to the estimated state of the feature of interest.

With the reasonable assumptions that the objective function is separable, i.e., that the terminal cost and stage cost functions are given by

$$
\begin{aligned}
\ell_{N}\left(P_{N \mid N}, \mathcal{I}_{N \mid N}, s_{N}\right) & =w_{s, u} \ell_{N}^{s}\left(s_{k}\right)+w_{P} \ell_{N}^{P}\left(P_{N \mid N}\right)+w_{\mathcal{I}} \ell_{N}^{\mathcal{I}}\left(\mathcal{I}_{N \mid N}\right), \\
\ell_{k}\left(P_{k \mid k}, \mathcal{I}_{k \mid k}, s_{k}, u_{k}\right) & =w_{s, u} \ell_{k}^{s, u}\left(s_{k}, u_{k}\right)+w_{P} \ell_{k}^{P}\left(P_{k \mid k}\right)+w_{\mathcal{I}} \ell_{k}^{\mathcal{I}}\left(\mathcal{I}_{k \mid k}\right),
\end{aligned}
$$

for $k=0, \ldots, N-1$, where $w_{s, u}, w_{P}$, and $w_{\mathcal{I}}$ are weighting parameters, and that both $\ell_{k}^{\mathcal{I}}$ and $\ell_{k}^{P}, k=0, \ldots, N$, are matrix decreasing on $\mathbf{S}_{++}^{n}$, the problem satisfies the requirements of Theorem 3.1, and the reformulations from the previous sections can be applied. Hence, the nonlinear equality constraints corresponding to the covariance matrix recursion can, as described in Section 3.4.2, be replaced by auxiliary decision variables and linear matrix inequality constraints, and the nonlinear equality constraints corresponding to the information matrix recursion can, as described in Section 3.3.2, be replaced by linear matrix inequalities. The stealthy IPP problem (3.66) is thus equivalent to a mixed-binary SDP problem that can be solved to global optimality using off-the-shelf branch and bound methods.

\section{Numerical illustrations}

This section presents results from simulations where the proposed method has been used to plan the path for a mobile sensor. The considered scenario involves a single feature of interest and one adversarial sensing region, and was selected to illustrate the concept and to demonstrate that intuitively sound trajectories are computed. The optimization problems are solved in Matlab 2017b using YALMIP's BnB solver [45], which uses MOSEK [3] to solve the convex relaxations.

In the simulations, the mobile sensor's dynamics are modeled as a discretized near-constant velocity model, where the state $s=\left[\begin{array}{llll}s_{1} & s_{2} & v_{1} & v_{2}\end{array}\right]^{\top}$ represents the mobile sensor's two-dimensional position and its velocity, and the control input $u$ 
corresponds to its acceleration. The set of admissible control inputs is given by $\mathcal{U}=\{u \mid-1 \preceq u \preceq 1\}$. The state of the feature is governed by a random walk model subject to white Gaussian noise with unit covariance. The adversarial observer estimates the state of the mobile sensor using measurements of its position, corrupted by white Gaussian measurement noise with unit covariance. A planning horizon of $N=6$ is used, and the terminal and stage cost functions are defined by

$$
\begin{aligned}
& \ell_{N}\left(P_{N \mid N}, \mathcal{I}_{N \mid N}, s_{N}\right)=w_{s, u} s_{N}^{\top} V_{s} s_{N}-w_{P} \operatorname{tr}\left(P_{N \mid N}\right)-w_{\mathcal{I}} \operatorname{tr}\left(\mathcal{I}_{N \mid N}\right), \\
& \ell_{k}\left(P_{k \mid k}, \mathcal{I}_{k \mid k}, s_{k}, u_{k}\right)=w_{s, u} s_{k}^{\top} V_{s} s_{k}-w_{P} \operatorname{tr}\left(P_{k \mid k}\right)-w_{\mathcal{I}} \operatorname{tr}\left(\mathcal{I}_{k \mid k}\right),
\end{aligned}
$$

for $k=0, \ldots, N-1$, where the weighting matrix $V_{s}=\operatorname{blkdiag}(0, I)$ is used to encode a penalty on the velocity of the mobile sensor and the terms are weighted with $w_{x, u}, w_{P}$, and $w_{\mathcal{I}}$.

In the first experiment, stealth and informativeness are weighted equally in the objective function, and there is a small penalty on velocity. The resulting trajectory is illustrated in Figure 3.6. The mobile sensor completely avoids entering the adversarial sensing region and is able to obtain three measurements of the feature of interest.

Results from a second experiment are shown in Figure 3.7. Here, information gathering has been prioritized by lowering the weight parameter on stealth. This results in a path through the adversarial sensing region, and gives away one measurement to the adversary, but the mobile sensor is able to obtain a fourth measurement of the feature of interest.

\subsection{Summary}

In this chapter, we have studied linear Gaussian IPP problems, for which the optimal sensor trajectory can be determined independently of the future measurement values. The main application was trajectory planning for a mobile sensor that is monitoring the evolution of features of interest in the environment. We have showed that under certain conditions, it is possible to reformulate seemingly intractable IPP problems as problems that reliably can be solved to global optimality using off-the-shelf optimization tools. Finally, we included the task of avoiding adversarial observers to the problem formulation, and proposed a method that allows for a trade-off between stealth and path informativeness when computing globally optimal sensor trajectories. 

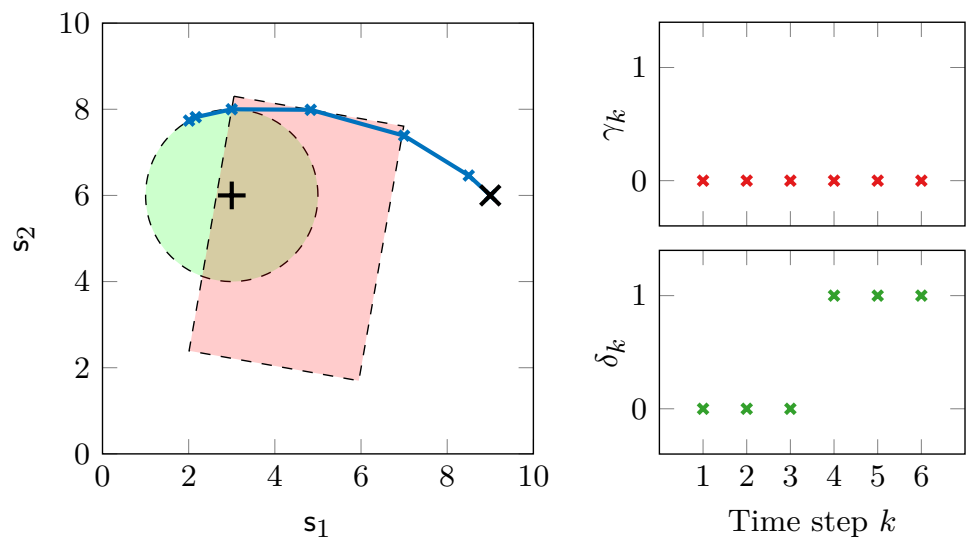

Figure 3.6: The mobile sensor starts at rest at $(9,6)$. The feature of interest is located at $(3,6)$ and is visible to the mobile sensor if the sensor is located in the green area. The red area is the sensing region of the adversarial observer. The weight parameters of the objective function are set to equally prioritize stealth and information gathering, and a small penalty on velocity $\left(w_{\mathcal{I}}=1, w_{P}=1, w_{s, u}=0.01\right)$. Left: Computed path for the mobile sensor. Upper right: Binary variables indicating whether or not the adversarial observer obtains measurements. Lower right: Binary variables indicating whether or not the mobile sensor obtains measurements.
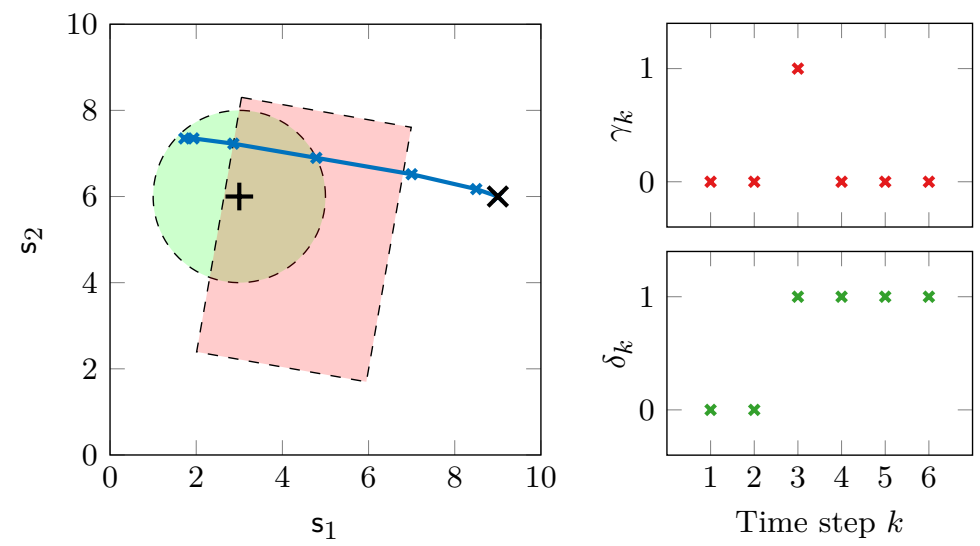

Figure 3.7: The setup is the same as in Figure 3.6, but the weight of stealth has been given a lower value $\left(w_{\mathcal{I}}=1, w_{P}=0.01, w_{s, u}=0.01\right)$. Left: Computed path for the mobile sensor. Upper right: Binary variables indicating whether or not the adversarial observer obtains measurements. Lower right: Binary variables indicating whether or not the mobile sensor obtains measurements. 


\section{Informative path planning for active target tracking}

This chapter considers tracking of a maneuvering target using a mobile sensor with limited field of view. The problem is formulated as an IPP problem, where the goal is to generate a sensor trajectory that maximizes the tracking performance, captured by a measure of the covariance matrix of the target state estimate. When the measurements are nonlinear functions of the target state, the tracking performance depends on the actual measurements, which are unavailable in the planning stage. Hence, the separation principle from Chapter 3 does not hold and the planning problem becomes a stochastic optimal control problem, where the expected tracking performance is used in the objective function. An approximation of the problem based on deterministic sampling of the distribution of the predicted target trajectory is proposed. It is shown that the proposed method significantly improves the ability to track maneuvering targets, compared to a baseline approach.

\subsection{Problem formulation}

In this chapter, we consider the problem of tracking a maneuvering target using a mobile sensor with limited field of view. This is formulated as a stochastic IPP problem with a measure of the expected tracking accuracy as objective function, and where models of the measurements, as well as the platform and the target, become constraints, as outlined below.

\subsubsection{Motion and measurement models}

Consider a mobile sensor, where the dynamics are governed by the following model,

$$
s_{k+1}=g\left(s_{k}, p_{k}\right)
$$


where $s_{k}$ is the state of the mobile sensor and $p_{k}$ is the applied motion primitive at time $k$. In this chapter, we will consider a lattice-based planning algorithm. The state of the mobile sensor is thus restricted to a discrete set of states, $s_{k} \in \mathcal{S}$, and the motion primitive, which is a sequence of control inputs, must be selected from the discrete set of applicable motion primitives at the state $s_{k}$, denoted $\mathcal{P}\left(s_{k}\right)$.

The motion of the target is assumed random, and it is modeled by the stochastic motion model

$$
x_{k+1}=f\left(x_{k}\right)+w_{k}, \quad w_{k} \sim \mathcal{N}(0, Q),
$$

where $x_{k}$ represents the state of the target and $w_{k}$ is the process noise, which is assumed to be white and Gaussian, with covariance $Q$ and zero mean.

The sensor is assumed to have limited field of view and sensing range. If the target is visible to the sensor at time $k$, an imperfect observation of the target is obtained according to

$$
y_{k}=h\left(x_{k}, s_{k}\right)+e_{k}, \quad e_{k} \sim \mathcal{N}(0, R),
$$

where $y_{k}$ is the measurement obtained by the sensor and $e_{k}$ is additive white measurement noise, which is assumed to be Gaussian with covariance $R$ and zero mean.

\subsubsection{Information representation}

The state of the target is tracked using an EKF, of which the main steps are outlined in Section 2.1.3. An important difference from the linear version of the Kalman filter is that the covariance matrix in an EKF depends on the actual measurements

and cannot be determined in advance. This is due to the linearization of the measurement equation and dynamics around the current state, which in turn depends on the measurement values. For notational convenience, the time and measurement update steps for the state estimate and corresponding covariance matrix are abbreviated as

$$
\begin{aligned}
& \hat{x}_{k+1 \mid k+1}=f_{x}\left(\hat{x}_{k \mid k}, P_{k \mid k}, s_{k+1}, y_{k+1}\right), \\
& P_{k+1 \mid k+1}=f_{P}\left(\hat{x}_{k \mid k}, P_{k \mid k}, s_{k+1}\right),
\end{aligned}
$$

where the measurement update is omitted if there is no measurement available at time $k+1$.

\subsubsection{Optimization problem}

The goal of the considered planning problem is to generate a sensor trajectory that maximizes the tracking performance. Since an EKF is used to track the target, an indication of the tracking performance can be obtained by a measure of the resulting covariance matrix of the target state estimate. Ignoring costs related to the motion of the mobile sensor, the objective function can be written as

$$
J_{N}=\sum_{k=0}^{N} \ell_{k}\left(P_{k \mid k}\right),
$$


where $N$ is the planning horizon. Appropriate choices of the stage cost functions $\ell_{k}$ are discussed in Section 2.1.5.

With the objective function and models outlined above, the problem of computing the optimal control policy $\pi=\left\{\mu_{0}, \ldots, \mu_{N-1}\right\}$, where $\mu_{k}$ gives the optimal motion primitive given the current output from the EKF and the state of the mobile sensor, can be formulated as an instance of the stochastic IPP problem (2.54):

$$
\begin{array}{ll}
\underset{\pi}{\operatorname{minimize}} & \mathbf{E}\left\{\sum_{k=0}^{N} \ell_{k}\left(P_{k \mid k}\right)\right\} \\
\text { subject to } & s_{k+1}=g\left(s_{k}, p_{k}\right) \\
& x_{k+1}=f\left(x_{k}\right)+w_{k} \\
& y_{k}=h\left(x_{k}, s_{k}\right)+e_{k} \\
& \hat{x}_{k+1 \mid k+1}=f_{x}\left(\hat{x}_{k \mid k}, P_{k \mid k}, s_{k+1}, y_{k+1}\right) \\
& P_{k+1 \mid k+1}=f_{P}\left(\hat{x}_{k \mid k}, P_{k \mid k}, s_{k+1}\right) \\
& p_{k}=\mu_{k}\left(\hat{x}_{k \mid k}, P_{k \mid k}, s_{k}\right) \in \mathcal{P}\left(s_{k}\right) \\
& s_{0}=\bar{s}_{0}, \quad s_{k} \in \mathcal{S},
\end{array}
$$

where $\bar{s}_{0}$ is the initial state of the mobile sensor, the expectation is taken with respect to the joint distribution of all the involved random variables, i.e., the target state trajectory, the measurement noise, and the prior distribution of the target state estimate, which is specified by $\mathcal{N}\left(\hat{x}_{0 \mid 0}, P_{0 \mid 0}\right)$.

The following sections propose methods to solve the planning problem using approximations based on deterministic sampling of the distribution of predicted target state trajectories.

\subsection{Approximations}

Instead of explicitly finding the optimal closed-loop policy by solving (4.6), we employ a standard receding horizon control scheme [46]. In each iteration of the receding horizon control loop, an open-loop optimal control problem, i.e., optimization over sequences of motion primitives instead of control policies $\pi$, is solved based on the current state of the mobile sensor and the current estimate of the state of the target. This gives an optimal sequence of motion primitives, $p_{k}, k=0, \ldots, N-1$, of which the first is applied to the mobile sensor. This moves the sensor to a different state from where it obtains a new measurement, and the target state estimate is updated using the EKF equations (4.4). In the next time step, the whole procedure is repeated with the new sensor state as $\bar{s}_{0}$ and the updated output from the EKF as $\mathcal{N}\left(\hat{x}_{0 \mid 0}, P_{0 \mid 0}\right)$, which leads to a standard receding horizon control loop.

The open-loop formulation of (4.6) is still a stochastic optimal control problem that is difficult to solve, since it requires prediction of states of nonlinear and noise-affected systems and involves an expectation operator in the objective function. This section develops approximations of the stochastic IPP problem based on sampling of the predicted target state distribution in different ways. First, a 
commonly used approach that will be used as baseline is presented. Following this, the approximation that is developed for this work is discussed.

\subsubsection{Baseline approach}

One way to handle stochastic systems in receding horizon control is to consider them as deterministic ones over the planning horizon [11]. The idea is then to repeatedly solve the problem and thus handle the uncertainty by the use of replanning. A straightforward approach is to replace stochastic quantities with their maximum likelihood values. For the considered problem, there are two sources of stochasticity: the uncertain dynamics of the target and the measurement noise. Thus, a reasonable approximation is to replace the stochastic target state by its maximum likelihood value $x_{k}^{\mathrm{ml}}=\arg \max p\left(x_{k}\right)$ and the stochastic observation $y_{k}$ by $y_{k}^{\mathrm{ml}}=\arg \max _{y} p\left(y \mid x_{k}^{\mathrm{ml}}, s_{k}\right)$. With these assumptions, the stochasticity in (4.4a) is eliminated and by that also the stochasticity in (4.4b), and the original problem is approximated by the following deterministic problem:

$$
\begin{array}{cl}
\underset{p_{0: N-1}}{\operatorname{minimize}} & \sum_{k=0}^{N} \ell_{k}\left(P_{k \mid k}\right) \\
\text { subject to } & s_{k+1}=g\left(s_{k}, p_{k}\right) \\
& \hat{x}_{k+1 \mid k+1}=f\left(\hat{x}_{k \mid k}\right) \\
& P_{k+1 \mid k+1}=f_{P}\left(\hat{x}_{k \mid k}, P_{k \mid k}, s_{k+1}\right) \\
& p_{k} \in \mathcal{P}\left(s_{k}\right), \quad s_{k} \in \mathcal{S} \\
& s_{0}=\bar{s}_{0}, \quad\left(\hat{x}_{0 \mid 0}, P_{0 \mid 0}\right)=\left(\overline{\hat{x}}_{0 \mid 0}, \bar{P}_{0 \mid 0}\right),
\end{array}
$$

where $\bar{s}_{0}$ is the initial state of the mobile sensor and $\left(\overline{\hat{x}}_{0 \mid 0}, \bar{P}_{0 \mid 0}\right)$ are the current state estimate and covariance matrix from the EKF.

The assumption of obtaining maximum likelihood observations corresponds to linearizing the measurement model around the predicted target trajectory during the planning phase and is commonly used in both IPP [4, 63] and motion planning under uncertainty $[53,57]$. This approximation will be used as a baseline approach in this work, and will thus be referred to as the baseline approach for the remainder of this chapter.

\subsubsection{Proposed approach}

A more advanced approximation than the baseline approach is to explicitly consider the stochasticity in the target state and use the maximum likelihood assumption only for the measurements. This approximation gives another stochastic optimal control problem, where the expectation is taken with respect to the target trajectory. This problem is still challenging, and finding a solution would require solving a complicated integral in each time step. However, numerical integration can be used as an approximation. By sampling the distribution of the predicted target state, a number of plausible target trajectories are obtained. These candidate target trajectories are then used to approximate the expectation with respect 
to the target state. This approximation yields the following deterministic optimization problem:

$$
\begin{array}{ll}
\underset{p_{0: N-1}}{\operatorname{minimize}} & \sum_{k=0}^{N} \sum_{i=1}^{L} w^{(i)} \ell_{k}\left(P_{k \mid k}^{(i)}\right) \\
\text { subject to } & s_{k+1}=g\left(s_{k}, p_{k}\right) \\
& y_{k}^{(i)}=h\left(\bar{x}_{k}^{(i)}, s_{k}\right) \\
& \hat{x}_{k+1 \mid k+1}^{(i)}=f_{x}\left(\hat{x}_{k \mid k}^{(i)}, P_{k \mid k}^{(i)}, s_{k+1}, y_{k+1}^{(i)}\right) \\
& P_{k+1 \mid k+1}^{(i)}=f_{P}\left(\hat{x}_{k \mid k}^{(i)}, P_{k \mid k}^{(i)}, s_{k+1}\right) \\
& p_{k} \in \mathcal{P}\left(s_{k}\right), \quad s_{k} \in \mathcal{S} \\
& s_{0}=\bar{s}_{0}, \quad\left(\hat{x}_{0 \mid 0}^{(i)}, P_{0 \mid 0}^{(i)}\right)=\left(\bar{x}_{0 \mid 0}, \bar{P}_{0 \mid 0}\right),
\end{array}
$$

where $L$ is the number of candidate target trajectories and superindex $(i)$ is used for entities related to the $i$ th candidate target trajectory: $\bar{x}_{k}^{(i)}$ is the state of the target at time $k$ given candidate trajectory $i$ and $w^{(i)}$ is the corresponding weight. The measurement at time $k$ corresponding to the $i$ th candidate trajectory is denoted $y_{k}^{(i)}$, and the resulting target state estimate and covariance matrix are denoted $\hat{x}_{k \mid k}^{(i)}$ and $P_{k \mid k}^{(i)}$, respectively. The approximated problem can be seen as a multiple-target problem; by considering each candidate target trajectory as a separate target, the optimal trajectory for the mobile sensor is the one that gives the best tracking performance in average for all targets.

The number of candidate target trajectories and the method for selecting them affect the accuracy of the approximation. If a large number of candidate trajectories are selected at random, the expectation with respect to the target trajectory is approximated using Monte Carlo integration [61]. If instead a few deterministically sampled trajectories are used to represent the distribution, the approach resembles the unscented transform [33]. If just a single candidate target trajectory is used and selected to be the most likely target trajectory, the method coincides with the baseline approach from [4].

As described above, there are several alternatives on how to select the candidate trajectories. The method used here is inspired by the unscented transform, and the candidate trajectories are generated through deterministic sampling of the distribution of predicted target state trajectories. In the standard form of the unscented transform, which is used in this work, $L=2 n_{x}+1$ carefully chosen samples, called sigma points, are used to represent the distribution. The parameter $n_{x}$ is the dimension of the state, and the representing sigma points $x^{(i)}$ and their corresponding weights $w^{(i)}$ are chosen as

$$
\begin{array}{ll}
x^{(0)}=\mathbf{E} x, & w^{(0)} \text { is a parameter, } \\
x^{( \pm i)}=x^{(0)} \pm \sqrt{\frac{n_{x}}{1-w^{(0)}}} z_{i}, & w^{( \pm i)}=\frac{1-w^{0}}{2 n_{x}},
\end{array}
$$




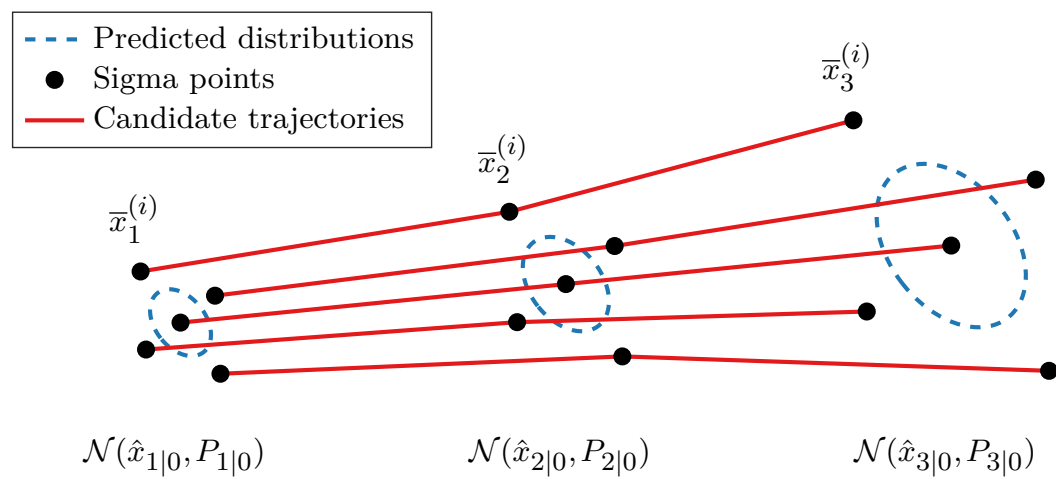

Figure 4.1: Conceptual illustration of how the candidate target trajectories are generated.

where $z_{i}$ is the $i$ th column in the square root of the covariance matrix of $x ; B$ is the a square root of $A$ if $A=B B^{\top}$.

The proposed method to generate candidate target trajectories consists of three steps: (i) prediction, (ii) sampling, and (iii) connection. The first step is to predict the a priori target state distribution for each time step within the planning horizon $N$. This generates the distributions $\mathcal{N}\left(\hat{x}_{k \mid 0}, P_{k \mid 0}\right), k=1, \ldots, N$. In the sampling step, the predicted distributions are sampled according to (4.9) in order to create sets of representing sigma points $\bar{x}_{k}^{(i)}$ and weights $w^{(i)}$. In the third step, the candidate target trajectories are created by connecting the matching sigma points. The process is illustrated in Figure 4.1.

\subsection{Graph search algorithm}

The solution to IPP problems with discrete sets of motion primitives can be obtained by enumerating all possible sensor trajectories and finding the one that results in the smallest objective function value. However, the number of possible trajectories increases exponentially with the planning horizon $N$ and the number of motion primitives, which makes the exhaustive search computationally intractable. This section presents methods that decrease the computational complexity of the search, without giving up guarantees on global optimality of the solution.

\subsubsection{Search tree generation}

As discussed in Section 2.3.3, classical motion planning using state-lattices involves a systematic search over a directed graph, where the nodes are the discrete states of the lattice $s_{k} \in \mathcal{S}$ and the edges are the motion primitives of $\mathcal{P}$ [56]. In the IPP setting, the aim is not to find a trajectory to a given goal state but rather to find a trajectory from where the sensor can obtain maximally informative measurements. Thus, each node also contains the accumulated objective function value $J_{k}$, which 


\section{Algorithm 4.1: Expand tree}

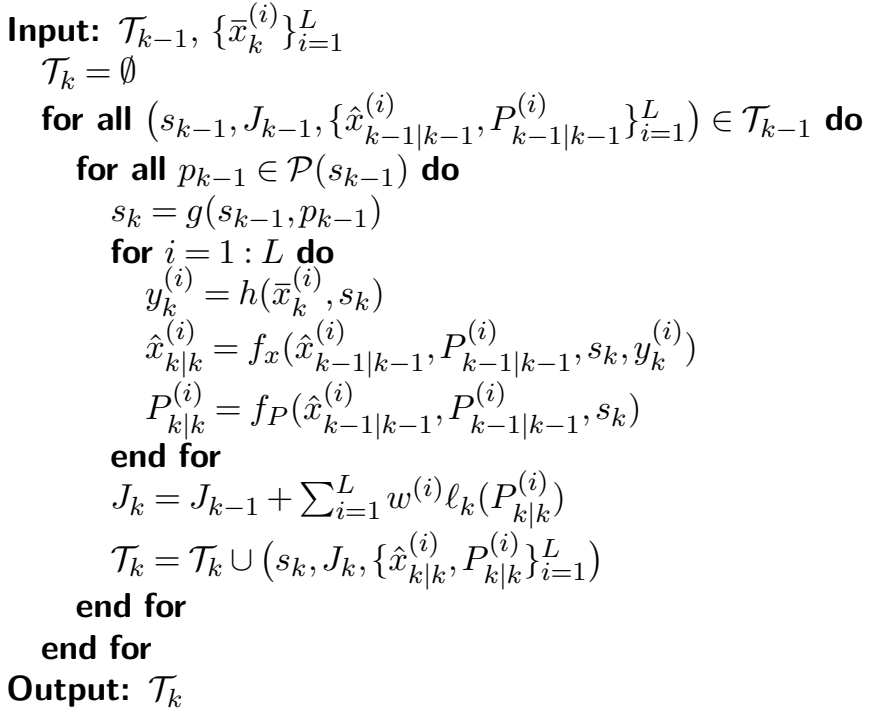

indicates the informativeness of the trajectory that leads to the node, as well as the target state estimate $\hat{x}_{k \mid k}^{(i)}$ and the corresponding covariance matrix $P_{k \mid k}^{(i)}$ for each of the candidate target trajectories. The graph, or search tree, is constructed using a forward search. A level of the tree is denoted $\mathcal{T}_{k}$ and defined as the set of nodes that can be reached by applying $k$ motion primitives from the initial state. Furthermore, let expand_tree denote the process of generating the set of nodes $\mathcal{T}_{k+1}$ by expanding the tree from level $\mathcal{T}_{k}$, i.e., applying every feasible motion primitive $p_{k} \in \mathcal{P}$ to each node $\left(s_{k}, J_{k},\left\{\hat{x}_{k \mid k}^{(i)}, P_{k \mid k}^{(i)}\right\}_{i=1}^{L}\right) \in \mathcal{T}_{k}$ in order to generate new nodes. The process is outlined in Algorithm 4.1.

By constructing the search tree, traditional systematic graph search methods can be applied to find the solution to the planning problem. The most straightforward approach is to perform an exhaustive search and form the search tree by sequentially applying expand_tree to its outermost level. The exhaustive search is guaranteed to find the optimal trajectory but suffers from exponential complexity since the number of nodes in the search tree corresponds to the number of trajectories of length $N$. Thus, this approach quickly gets intractable.

Traditional graph search methods typically use heuristic estimates of the remaining cost-to-go in order to increase the efficiency of the graph search [39]. For classical motion planning, where a goal state is specified, an intuitive heuristic is the remaining path length, e.g., approximated by the Euclidean distance to the goal. When the heuristic function is defined, algorithms like $A^{*}$ [54] can be used to avoid searching in parts of the tree that are unlikely to contain the optimal solution. For IPP problems it is more difficult to come up with a suitable heuristic function, since there is no well-defined goal state to which the distance could be used to estimate the remaining cost-to-go. Instead, the heuristic function would 


\section{Algorithm 4.2: Greedy search}

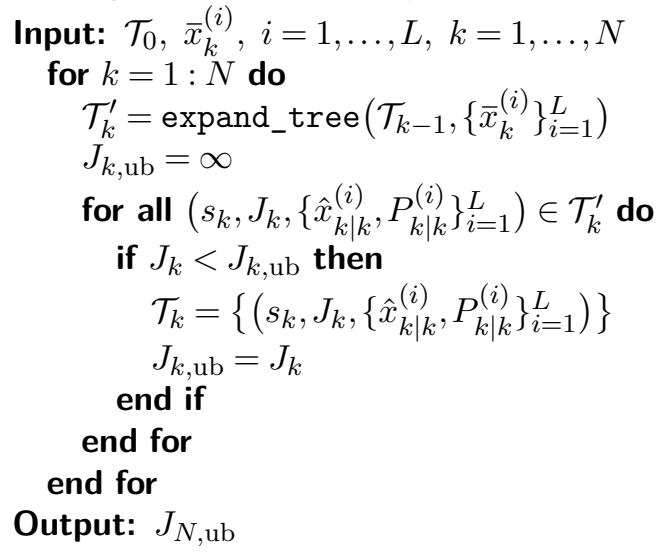

be used to approximate the amount of information that is possible to collect from the current state until the end of the planning horizon. An alternative to focusing the search using heuristics is to prune the search tree in order to reduce its size and thereby decrease the computational burden.

\subsubsection{Pruning strategies}

The search algorithm that is used in this work uses two types of pruning strategies. These are described in the following subsections.

\section{Pruning based on branch and bound}

The first pruning strategy has similarities to the branch and bound algorithm [23] in the sense that it is based on upper and lower bounds on the optimal objective function value. During the execution of the search algorithm, an upper bound on the globally optimal objective function value is maintained. As a new node is created, a lower bound on the optimal objective function value for a trajectory that contains the newly created node is computed. If the lower bound at a certain node is greater than the upper bound on the optimal objective function value, the subtree rooted at that particular node does not need to be further explored. The optimal sensor trajectory will not contain the node, since there must exist a better node in another part of the search tree, and the node can thus be pruned.

The first upper bound on the optimal objective function value is obtained using a greedy search method that is outlined in Algorithm 4.2. Each level of the search tree created with this greedy method contains just one single node - all nodes except the one with lowest objective function value are discarded when the level is created.

As the full search is conducted and new nodes are added, the upper bound may be improved. When a new node is created, an upper bound on the optimal objective function value for a trajectory that contains that particular node is computed. 
If the current upper bound on the optimal objective function value is greater than the new bound, it is replaced by the new bound. There are several ways to compute the upper bound, e.g., by performing a greedy search from the new node to the end of the planning horizon. Here, the upper bound is computed using a worst case scenario by evaluating the objective function as if no more measurements were to be obtained until the planning horizon.

The lower bound in branch and bound algorithms can be found in various ways, e.g., by convex relaxations of the original problem as in Chapter 3. In general, there is a trade-off between how tight a lower bound is and the effort needed to compute it. Here, a simple method is used to find the lower bound: assuming that the stage cost function is non-negative, a lower bound on the optimal objective function value for a trajectory that contains a certain node is the accumulated cost at that particular node. If the trace of the covariance matrix is used as the stage cost function, this corresponds to assuming that the state of the target will be perfectly known for the remaining time steps.

\section{Pruning based on algebraic redundancy}

The second pruning strategy is based on the ideas in [70]. If there are several trajectories that end up in the same state of the mobile sensor at the same level of the search tree, but yield different covariance matrices, the trajectories that result in clearly less informative measurements are considered redundant and can be discarded. This pruning strategy is the main result of [70], where the idea of redundancy is formalized using the following definition and theorem:

Definition 4.1 (Algebraic redundancy [70]). A node $\left(s, J,\left\{\hat{x}^{(i)}, P^{(i)}\right\}_{i=1}^{L}\right)$ is algebraically redundant with respect to the set $\left\{\left(s_{j}, J_{j},\left\{\hat{x}_{j}^{(i)}, P_{j}^{(i)}\right\}_{i=1}^{L}\right)\right\}_{j=1}^{K}$ if there, for $i=1, \ldots, L$, exist nonnegative constants $\alpha_{j}^{(i)}$ such that

$$
\sum_{j \text { s.t. } s_{j}=s} \alpha_{j}^{(i)}=1, \quad \text { and } \quad\left[\begin{array}{cc}
P^{(i)} & 0 \\
0 & J
\end{array}\right] \succeq \sum_{j \text { s.t. } s=s_{j}} \alpha_{j}^{(i)}\left[\begin{array}{cc}
P_{j}^{(i)} & 0 \\
0 & J_{j}
\end{array}\right] \text {. }
$$

Theorem 4.1 (Algebraic redundancy pruning [70]). Let $\mathcal{T}$ be a set of nodes at the same level of the search tree. If the node $A \in \mathcal{T}$ is algebraically redundant with respect to $\mathcal{T} \backslash A$, then the node $A$ and all of its descendants can be pruned without eliminating the optimal solution from the search tree.

By applying Theorem 4.1, algebraically redundant nodes can be pruned from the search tree without the risk of eliminating the optimal trajectory.

\subsubsection{Resulting algorithm}

By combining the two pruning strategies presented in the previous section, it is possible to reduce the number of nodes that need to be explored in order to find the optimal solution to the graph search problem. As illustrated in Figure 4.2, 


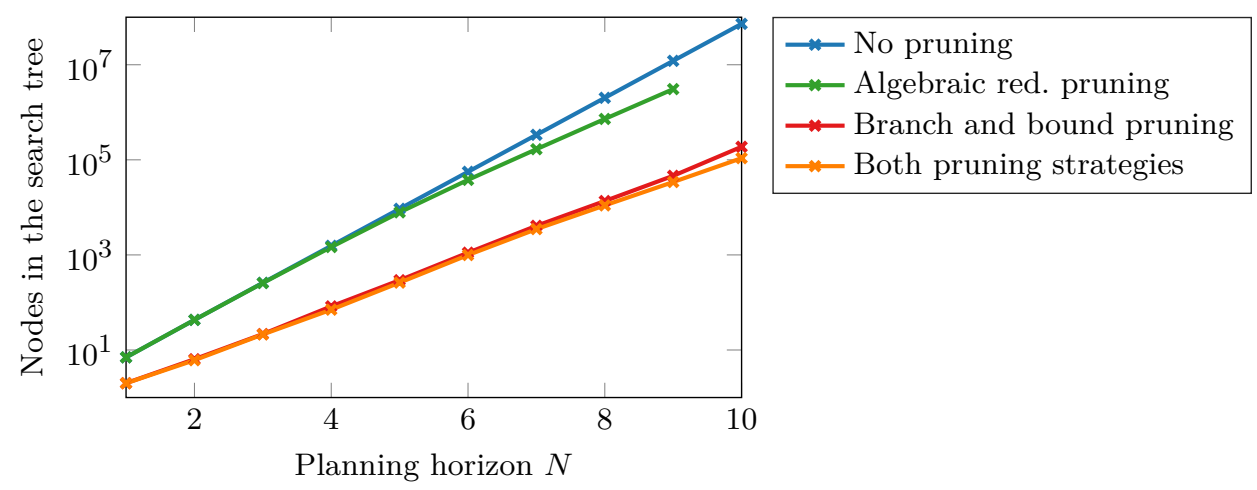

Figure 4.2: Total number of nodes in the search tree for different pruning strategies and planning horizons.

the branch and bound pruning eliminates far more nodes than the algebraic redundancy pruning. The main reason for this is that the algebraic redundancy pruning strategy only compares nodes that correspond to the same state of the mobile sensor, while the branch and bound pruning strategy considers all nodes at a given level of the tree. The resulting algorithm for constructing the search tree is outlined in Algorithm 4.3.

\section{Algorithm 4.3: Graph search}

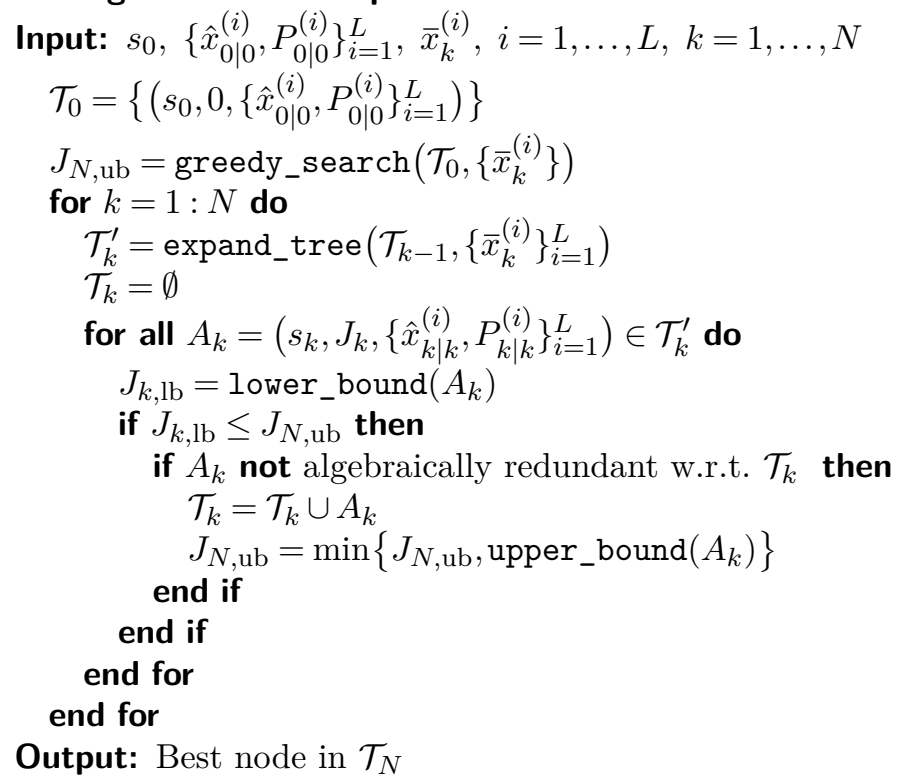




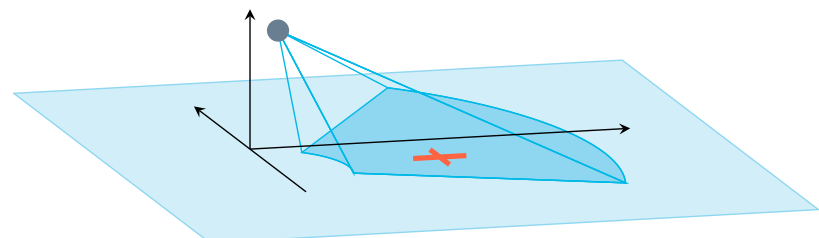

Figure 4.3: Illustration of the sensor's limited field of view. Targets located in the darker area are visible to the sensor.

\subsection{Simulation study}

In this section, the planning approaches are evaluated on a number of target tracking scenarios. The aim of the simulation study is to evaluate the effects of varying target agility, planning horizon, and robustness to different measurement noise levels.

\subsubsection{Simulation setup}

The true target moves on the ground according to a differential drive model with varying angular rate $\omega$ and constant speed of five meters per seconds. For planning and estimation, the target state is modeled by its two-dimensional position and velocity, $x=\left[\begin{array}{llll}\mathrm{x}_{1} & \mathrm{x}_{2} & \mathrm{v}_{1} & \mathrm{v}_{2}\end{array}\right]^{\top}$, and a near-constant velocity motion model driven by Gaussian white noise, discretized with a sampling period $\tau=0.5 \mathrm{~s}$. That is,

$$
x_{k+1}=\left[\begin{array}{cc}
I & \tau I \\
0 & I
\end{array}\right] x_{k}+w_{k}, \quad w_{k} \sim \mathcal{N}\left(0, q\left[\begin{array}{cc}
\tau^{3} / 3 I & \tau^{2} / 2 I \\
\tau^{2} / 2 I & \tau I
\end{array}\right]\right) .
$$

The mobile sensor moves according to differential drive dynamics at a constant altitude of 20 meters, and the state consists of its two-dimensional position, heading angle, and speed, $s=\left[\begin{array}{llll}\mathrm{s}_{1} & \mathrm{~s}_{2} & \theta & v\end{array}\right]^{\top}$. The center of the field of view is fixed to the heading angle. When applying the state-lattice approach, the state space needs to be discretized. For the experiments, the state-space discretization is made such that the discretization accuracy of the position is 0.5 meter for both $\mathrm{s}_{1}$ and $\mathrm{s}_{2}$, the heading angle is discretized into 16 different angles and $v \in\{4,6\} \mathrm{m} / \mathrm{s}$. The motion primitives are generated using ACADO [29] and satisfy the dynamic constraints of the motion model, which makes it possible to guarantee that the planned trajectory will be dynamically feasible for the mobile sensor.

The mobile sensor carries a lidar-like sensor with limited field of view and limited sensing range, as illustrated in Figure 4.3. If the target is visible to the sensor, the obtained measurement consists of the horizontal range and bearing from the sensor to the target:

$$
\begin{aligned}
y_{k} & =h\left(x_{k}, s_{k}\right)+e_{k}, \quad e_{k} \sim \mathcal{N}(0, R), \\
h(x, s) & =\left[\begin{array}{l}
r(x, s) \\
\alpha(x, s)
\end{array}\right]=\left[\begin{array}{c}
\sqrt{\left(\mathrm{x}_{1}-\mathrm{s}_{1}\right)^{2}+\left(\mathrm{x}_{2}-\mathrm{s}_{2}\right)^{2}} \\
\arctan \left(\left(\mathrm{x}_{2}-\mathrm{s}_{2}\right) /\left(\mathrm{x}_{1}-\mathrm{s}_{1}\right)\right)
\end{array}\right] .
\end{aligned}
$$




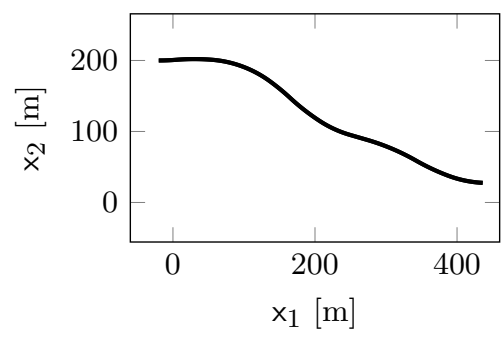

(a) Slowly maneuvering target

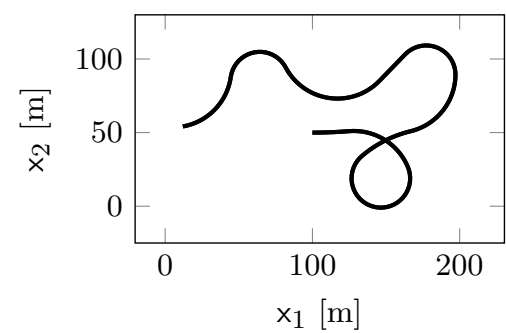

(b) Agile target

Figure 4.4: Target trajectories considered in the experiments.

where the measurement noise $e_{k}$ is assumed to be white and Gaussian with covariance $R$. Note that even though the magnitude of the measurement noise, given by the constant covariance matrix $R$, is independent of the current state $s_{k}$ of the sensor, the Jacobian of the measurement equation is given by

$$
H=\frac{1}{r(x, s)}\left[\begin{array}{cccc}
\left(\mathrm{x}_{1}-\mathrm{s}_{1}\right) & \left(\mathrm{x}_{2}-\mathrm{s}_{2}\right) & 0 & 0 \\
-\sin (\alpha(x, s)) & \cos (\alpha(x, s)) & 0 & 0
\end{array}\right],
$$

which means that the information contained in a measurement, given by $H^{\top} R^{-1} H$, is inversely proportional to the distance between sensor and target. Furthermore, note that the information cannot increase indefinitely due to the sensor footprint, illustrated in Figure 4.3, which limits the minimum range for a measurement. Since the magnitude of the covariance of the target state estimate is tightly connected to the amount of information collected and the aim is to reduce the covariance as much as possible, the sensor should position itself such that the target is as close as possible, but still within field of view.

\subsubsection{Findings}

The proposed method is evaluated and compared to the baseline approach using Monte Carlo simulations of different relevant tracking scenarios. The statistics are generated from 1000 simulations for each experiment. In all simulations, the stage cost is the trace of the covariance matrix: $\ell_{k}(P)=\operatorname{tr}(P)$, and the sampling period is $\tau=0.5 \mathrm{~s}$. The performance metrics are: (i) the ability to maintain track of the target, (ii) the root mean square error (RMSE) of the estimated target position, and (iii) the computational complexity of the planning algorithms.

\section{Choice of planning horizon}

The first set of experiments investigates how the planning horizon affects the performance. Two different scenarios are considered, both illustrated in Figure 4.4, in order to determine the impact of the planning horizon for different target motions. For all simulations, the parameter $q$ is set to 0.1 and the sensor parameters are 

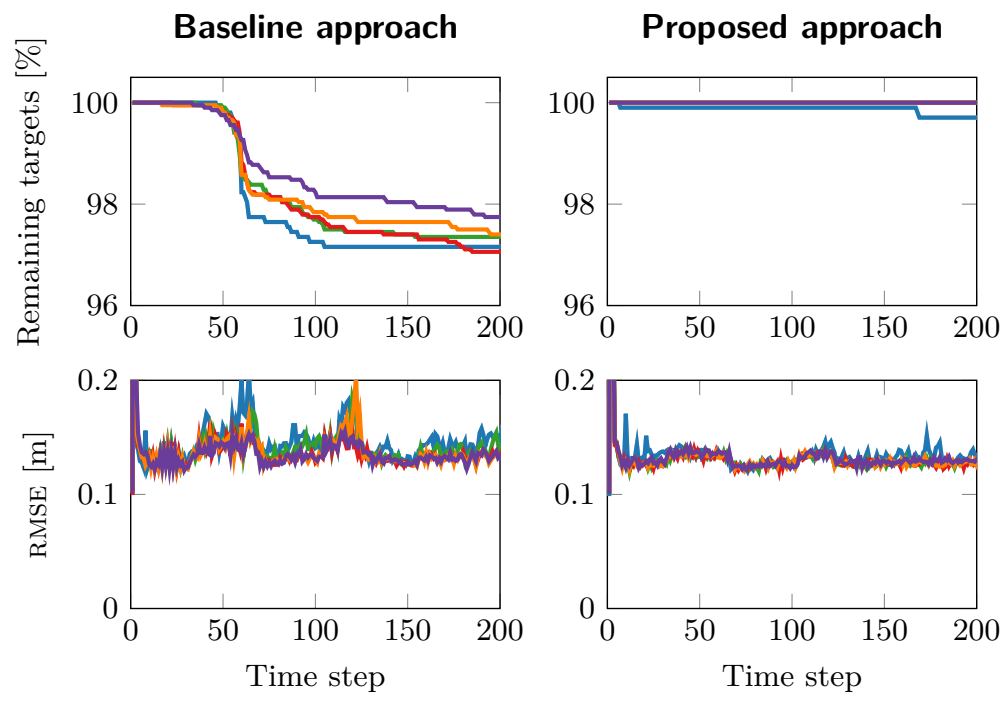

$-N=1-N=2-N=4-N=6-N=8$

Figure 4.5: Results for active tracking of a slowly maneuvering target with trajectory as in Figure 4.4a.

set to model a high-quality lidar, inspired by [42]: the standard deviation of the range and bearing measurements are $\sigma_{r}=0.05 \mathrm{~m}$ and $\sigma_{\alpha}=0.5^{\circ}$, respectively.

In the first scenario, the target is slowly maneuvering and follows an almost straight path, which means that the near-constant velocity model is a fairly accurate description of target motion. This means that the maximum-likelihood prediction of the target trajectory often coincides with the actual target trajectory. Thus, the baseline approach should perform well in this scenario.

The results from the simulations of this scenario are presented in Figure 4.5. As expected, the baseline approach performs well and the sensor loses track of the target in only about two percent of the simulations. The performance of the baseline approach also increases with longer planning horizon, which is expected since the target behaves as the model predicts. However, it is still outperformed by the proposed method, which manages to maintain track of the target in almost all simulations.

In the second scenario, an agile target is performing sharp turns and is thus constantly deviating from the trajectory predicted by the constant velocity motion model. Since the baseline approach does not take uncertainty in the target trajectory into account, it is expected to perform worse than it did in the previous scenario. The proposed approach on the other hand takes this uncertainty into account, and should be able to handle also agile targets. These predictions are confirmed by the simulations, of which the results are presented in Figure 4.6. The proposed approach is able to maintain track of the target in almost every 

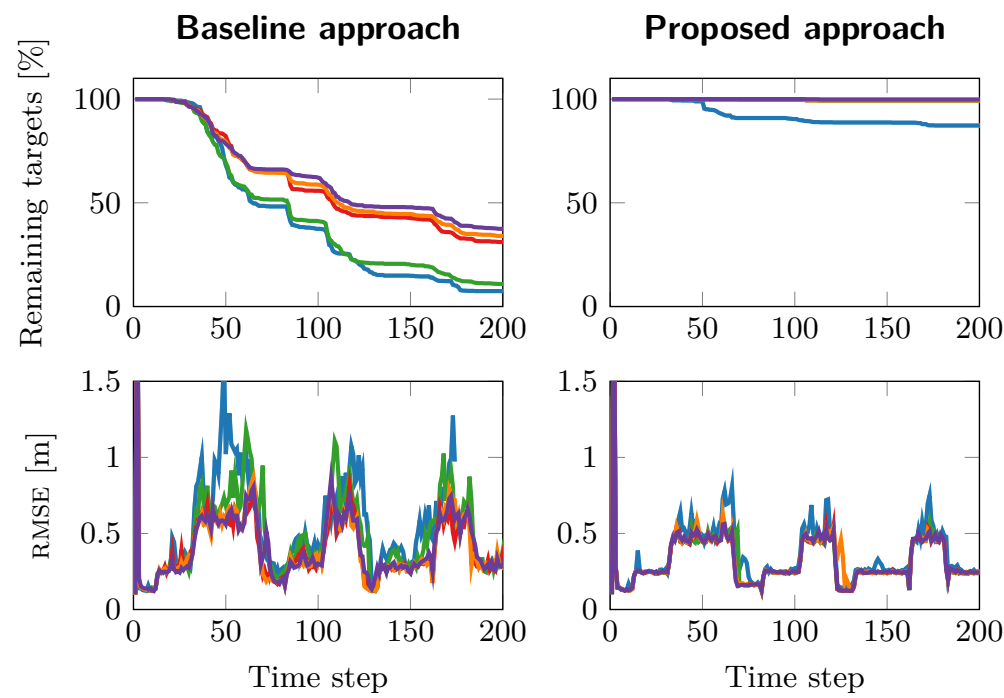

$$
-N=1-N=2-N=4-N=6-N=8
$$

Figure 4.6: Results for active tracking of an agile target with trajectory as in Figure $4.4 b$.

simulation, provided that the planning horizon is longer than just a single time step. The baseline approach performs poorly for this scenario and even though the performance is increased by increasing the planning horizon, the agile target is still lost in more than $50 \%$ of the simulations after 200 time steps.

Overall, the proposed approach is significantly less sensitive to the choice of the planning horizon $N$. Even though a planning horizon of two steps would be enough for the proposed approach, $N=6$ will be used for the remainder of the experiments since it gives reasonable performance also for the baseline approach.

\section{Target agility}

The next set of experiments investigates how well the planning algorithms are able to handle target trajectories of varying agility. We let the angular rate $\omega$ reflect the target's agility level and test the performance for the target trajectories that are illustrated in Figure 4.7. The trajectory with largest angular rate, $\omega=\pi / 12.5 \mathrm{rad} / \mathrm{s}$, corresponds to the minimum turning radius for the sensor platform. For all simulations, the planning horizon is $N=6$ and the standard deviation of the range and bearing measurements are $\sigma_{r}=0.05 \mathrm{~m}$ and $\sigma_{\alpha}=0.5^{\circ}$, respectively.

The tuning parameter $q$ reflects the expected agility of the target. A high value of $q$ allows for tracking a highly maneuvering target. To evaluate how sensitive the algorithms are to the tuning of this parameter, the simulations are run for three different values of $q$ : (i) a low value $(q=0.01)$, representative for target trajectories where the magnitude of the angular rate $\omega$ is less than $\pi / 100 \mathrm{rad} / \mathrm{s}$; 


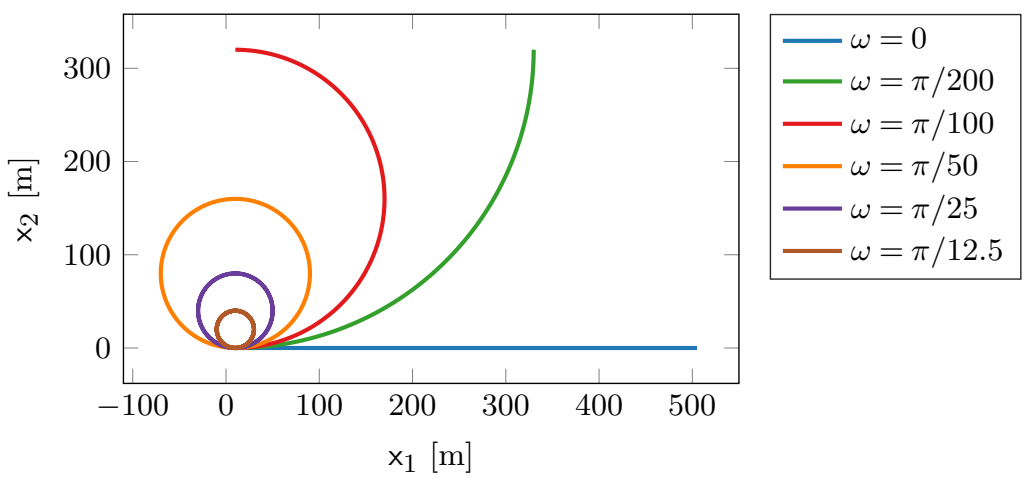

Figure 4.7: Target trajectories for different angular rates $\omega$.

(ii) a "normal" value $(q=0.1)$, that could be considered as a reasonable tuning for tracking of targets with trajectories as in Figure 4.7; and (iii) a high value $(q=1)$, which would be appropriate to use for targets that turn more quickly than in the considered trajectories. The results from this set of experiments are presented in Figures 4.8 and 4.9 .

The sensor's ability to maintain track of the target is shown in Figure 4.8. The baseline approach is able to handle slowly maneuvering targets, i.e., trajectories with small angular rate $\omega$, if the parameter $q$ is tuned appropriately. In other cases, it generates sensor trajectories that eventually lead to losing track of the target. If the parameter $q$ is set to a high value, the latest measurement greatly affects the current target state estimate. Since the baseline approach plans the sensor trajectory based only on the most likely target trajectory given the current estimate of the target state, a noisy measurement could send the sensor in a direction that leads to a lost track.

By taking the uncertainty in the target trajectory into account, the proposed approach significantly improves the ability to maintain track of the target. As shown in the second column of Figure 4.8, the target is essentially not lost in any of the simulations except for the case when the value of the parameter $q$ is significantly too small. Compared to the baseline approach, the proposed approach is significantly more robust to the tuning of the parameter $q$ and avoids losing track of the target. In particular, the method copes with higher agility than expected much better than the baseline approach. This allows for tuning for the nominal case while still being able to handle extreme cases.

Figure 4.9 shows the tracking accuracy of the tracked targets. Note that only targets that are tracked are taken into account when calculating the RMSE. By comparing the first column with the second column of Figure 4.9, it is evident that the position RMSE for the remaining targets are approximately the same for both approaches. Thus, the increased robustness of the proposed approach does not come at the price of reduced tracking accuracy in terms of RMSE. 


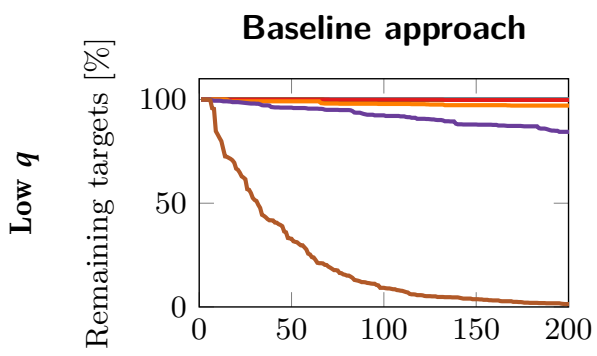

Proposed approach
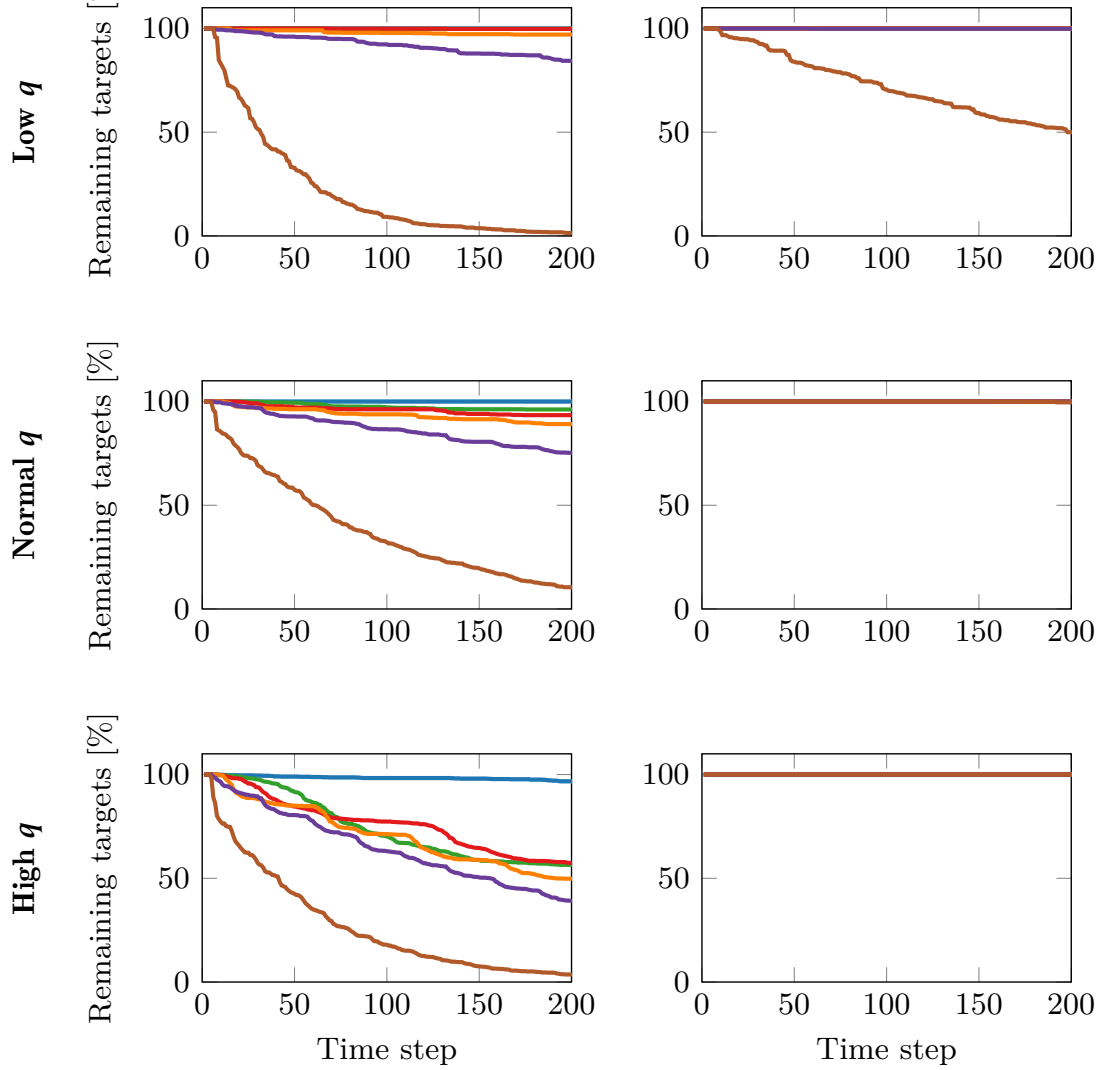

$$
\begin{array}{lllll}
\omega=0 & -\omega=\pi / 200 & \omega & \omega=\pi / 100 \\
\omega=\pi / 50 & \omega=\pi / 25 & \omega & \omega=\pi / 12.5
\end{array}
$$

Figure 4.8: Percentage of the simulations in which the sensor is able to keep track of the target for different target turning rates. 

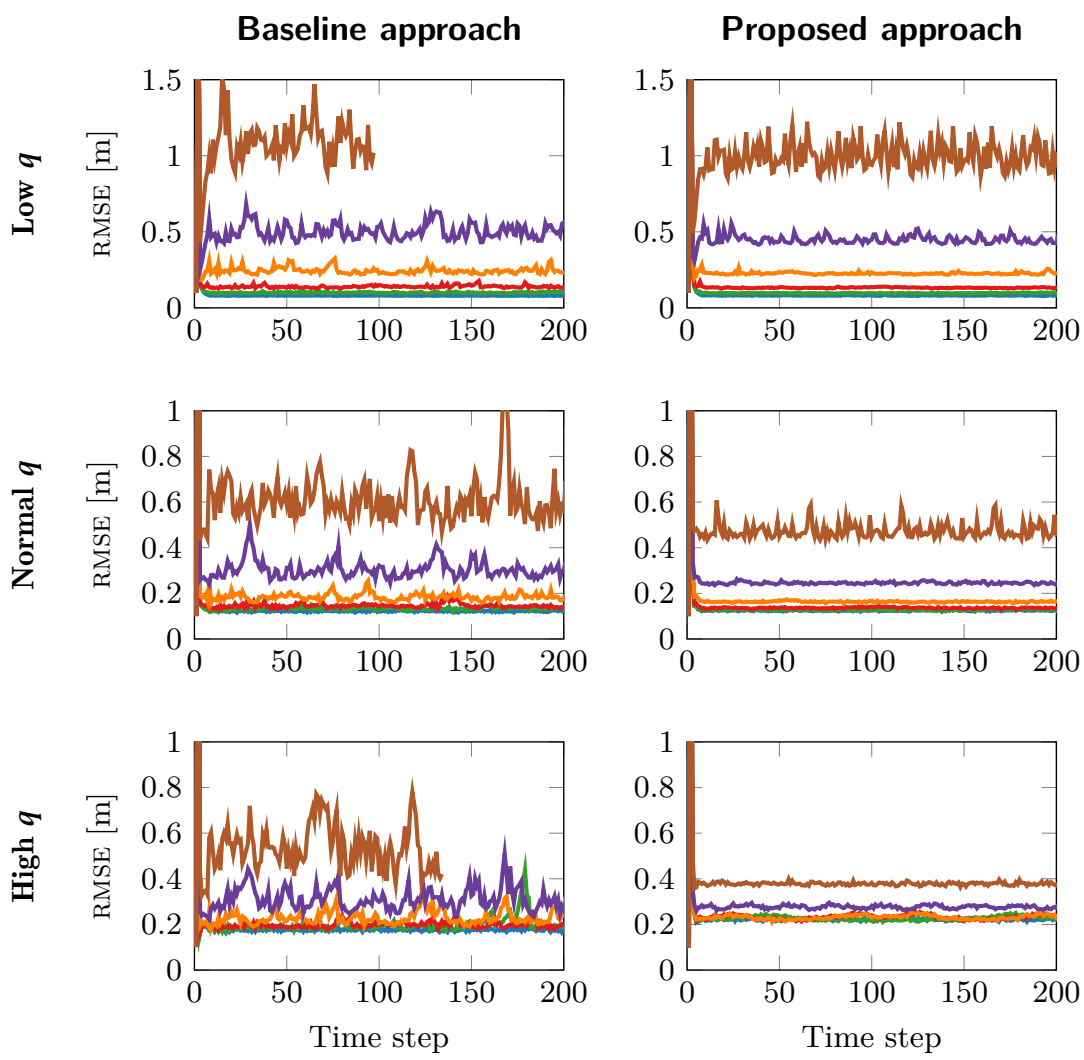

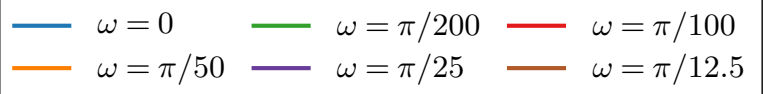

Figure 4.9: RMSE of the estimated target position for different target turning rates. 

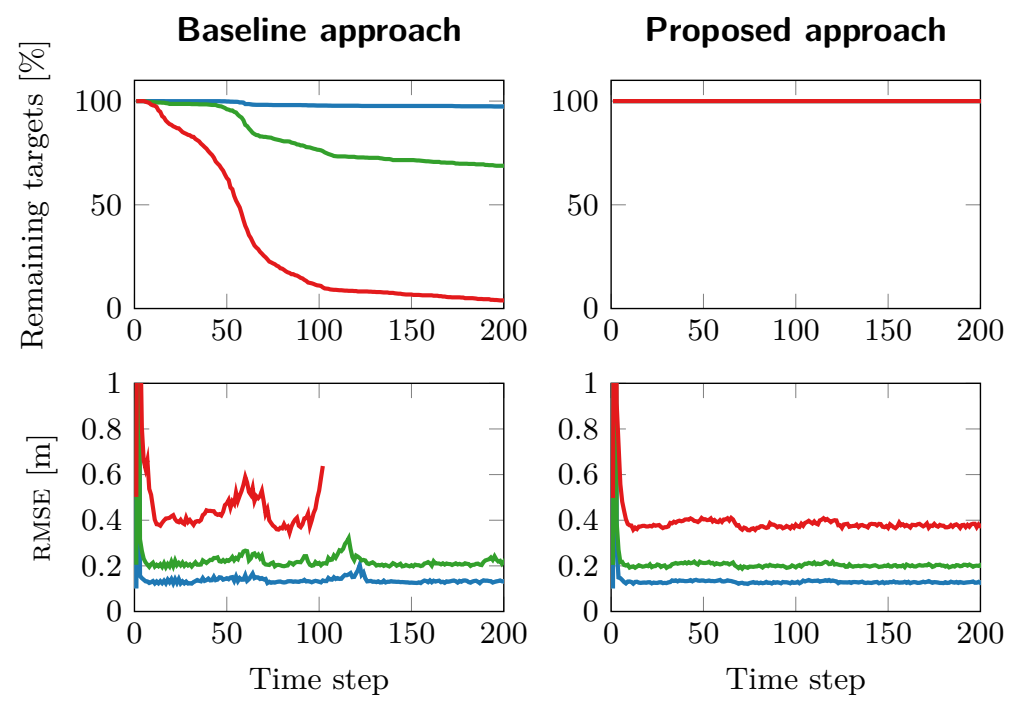

$$
\begin{aligned}
-\sigma_{r}=0.05 \mathrm{~m}, & \sigma_{\alpha}=0.5^{\circ} \\
-\sigma_{r}=0.1 \mathrm{~m}, & \sigma_{\alpha}=1.0^{\circ} \\
-\sigma_{r}=0.25 \mathrm{~m}, & \sigma_{\alpha}=2.5^{\circ}
\end{aligned}
$$

Figure 4.10: Results for active tracking of a slowly maneuvering target with trajectory as in Figure 4.4a.

\section{Measurement noise}

This set of experiments investigates how the magnitude of the measurement noise affects the performance of the planning algorithms. This is tested by simulating the scenarios illustrated in Figure 4.4 using different values for the standard deviation of the measurement noise. The noise levels are reasonable for lidars, and not greater than what the baseline approach can handle. For all simulations, the planning horizon is $N=6$ and the parameter $q$ is set to 0.1 . The results are presented in Figures 4.10 and 4.11 .

The ability to keep track of the target degrades with increasing level of measurement noise, but the proposed approach is more robust than the baseline approach. The baseline approach depends on having an accurate estimate of the target's velocity, since it does not take uncertainty into account. The velocity is not measured directly, but estimated through measurements of the target's position, which means that the accuracy of this estimate rapidly decreases as the magnitude of the measurement noise increases. Furthermore, for both scenarios and planning approaches, the performance in terms of RMSE degrades with increasing level of measurement noise, as might be expected. 

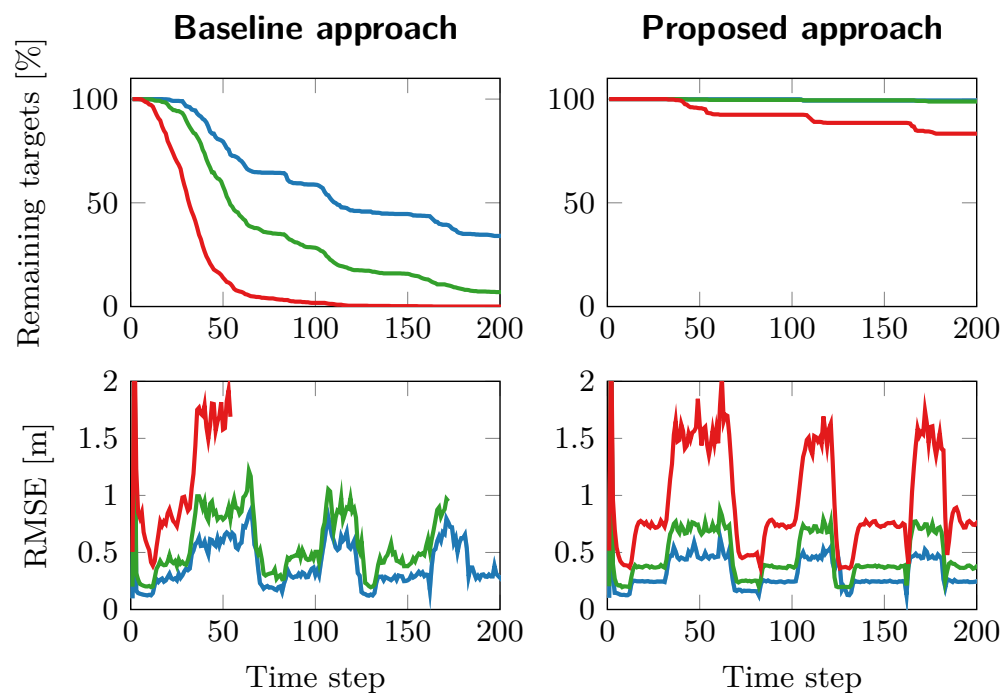

$$
\begin{aligned}
-\sigma_{r}=0.05 \mathrm{~m}, & \sigma_{\alpha}=0.5^{\circ} \\
-\sigma_{r}=0.1 \mathrm{~m}, & \sigma_{\alpha}=1.0^{\circ} \\
-\sigma_{r}=0.25 \mathrm{~m}, & \sigma_{\alpha}=2.5^{\circ}
\end{aligned}
$$

Figure 4.11: Results for active tracking of an agile target with trajectory as in Figure $4.4 b$.

\section{Computational complexity}

As new measurements are obtained, it is necessary to re-plan the sensor trajectory online to adapt to the newly acquired information. Hence, the time needed to find a solution to the graph search problem is of interest if the planning algorithm is to be used in real-time applications. To investigate this, the algorithms have been naively implemented in $\mathrm{C}++$, with little effort to optimize the code, and simulations run on a laptop with a $2.6 \mathrm{GHz}$ Intel i7 processor with $16 \mathrm{~GB}$ of RAM running Ubuntu 16.04. Figure 4.12 reveals that the computational complexity of both approaches grow rapidly with the planning horizon and that the proposed approach is, not surprisingly, computationally heavier than the baseline approach. However, recall that the proposed approach performs significantly better than the baseline approach even when using a shorter planning horizon. Furthermore, when applying a receding horizon approach, the next plan can be computed during execution of the first motion primitive of the current plan. In this case, the duration of all motion primitives are $0.5 \mathrm{~s}$, which means that even this rudimentary implementation of the proposed approach is fast enough for use in real-time applications when using a planning horizon of less than eight time steps. 


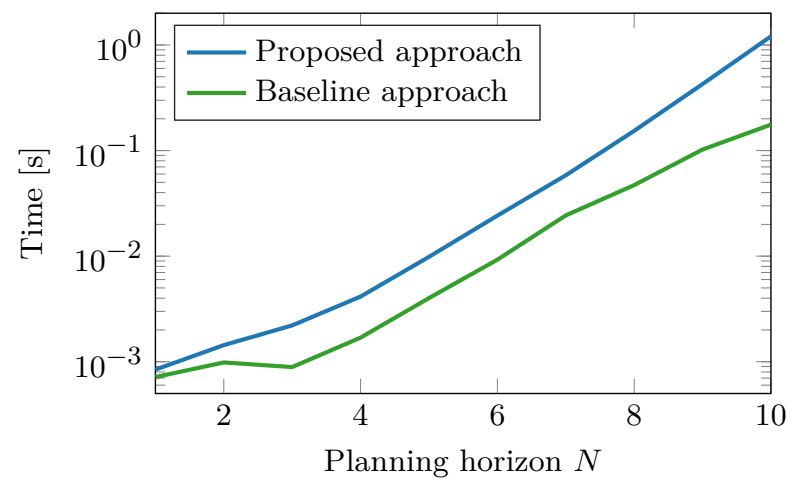

Figure 4.12: Average computation time needed to find the solution to the graph search problem for different planning horizons.

\subsection{Summary}

In this chapter, we have considered the problem of active target tracking and formulated it as a nonlinear stochastic IPP problem. An approximate planning method, that takes the uncertainty in the future target trajectory into account by deterministic sampling of the a priori distributions, has been proposed. As demonstrated in a simulation study, the proposed method significantly increases the tracking performance compared to a conventional approach that neglects the uncertainty in the future target trajectory. In particular, the proposed method decreases the need for a long planning horizon and is less sensitive to tuning of the parameters related to target maneuverability and measurement noise. 


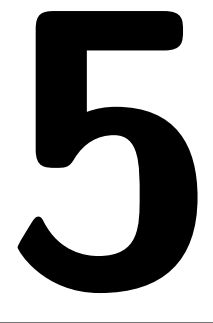

\section{Concluding remarks}

This chapter concludes the thesis by summarizing the results and discussing some possible directions for future work.

\subsection{Conclusions}

A fully automated sensor system requires the capability of deciding where to go to collect necessary information. Informative path planning (IPP), which has been studied in this thesis, is thus an important part of sensor management. Arguably, both informative path planning and sensor management are difficult problems. In general, problems in these areas can be formulated as nonlinear optimal control problems for stochastic systems.

Chapter 3 addressed the problem of providing optimality guarantees in informative path planning. As in many other areas, an assumption of linear Gaussian systems simplified things. Here, it reduced the stochastic optimal control problem to a deterministic one. Although the linear Gaussian problem is deterministic, it is both nonlinear and nonconvex, and still difficult to solve. Through a series of reformulations, it was shown that the considered problem is equivalent to a mixed binary SDP, that can reliably be solved to global optimality using off-the-shelf optimization tools. Furthermore, it was shown that also problems that include adversarial observers can be reformulated in a similar manner. The proposed approach allows for a trade-off between stealth and informativeness when computing globally optimal sensor trajectories.

Chapter 4 dealt with stochasticity in informative path planning. The considered scenario was an active target tracking problem, where the goal was to compute a sensor trajectory that maximizes the expected tracking performance. A receding horizon control strategy was employed, and the stochasticity was handled by deterministic sampling of the distribution of future target trajectories. This way, 
several candidate target trajectories were considered when optimizing the sensor trajectory. A simulation study showed that the proposed approach significantly increases the tracking performance compared to a baseline approach, where only the most likely target trajectory is considered.

\subsection{Future work}

This thesis has taken steps towards creating a sensor management system by optimizing the movement of the sensor platform. The work of this thesis can be extended in several ways.

One possible direction for future research is to improve the methods developed in this thesis, with the focus on a single sensor platform. The methodology proposed in Chapter 4 opens up for taking other aspects of the uncertainty into account, e.g., by using more advanced schemes for selecting the candidate target trajectories or considering the effects of different measurement noise realizations.

Another interesting topic for future research is to explicitly consider several sensor platforms in the problem formulation. Although the theoretical results of this thesis hold also for multiple-sensor systems, the focus has been on systems consisting of a single sensor. A possible extension is thus to develop informative path planning methods that are both scalable and decentralized, to be more suitable for multiple-platform settings. The multiple-platform case can be further extended by also letting each platform carry several heterogeneous sensors.

A third possible direction for future research is to move towards full-scale sensor management. This means including more degrees of freedom in the problem formulation, e.g., considering sensors with several different operational modes with varying level of accuracy, and constrained budgets that limit the use of certain sensors or the movement of the sensor platforms during a mission. 


\section{Bibliography}

[1] Mohamed Al Marzouqi and Ray A. Jarvis. Robotic covert path planning: A survey. In Proceedings of IEEE Conference on Robotics, Automation and Mechatronics, pages 77-82, Beijing, China, 2011.

[2] Olov Andersson, Oskar Ljungqvist, Mattias Tiger, Daniel Axehill, and Fredrik Heintz. Receding-horizon lattice-based motion planning with dynamic obstacle avoidance. In Proceedings of the 57th IEEE Conference on Decision and Control, pages 4467-4474, Miami Beach, FL, USA, 2018.

[3] MOSEK ApS. The MOSEK optimization toolbox for MATLAB manual. Version 8.1., 2017.

[4] Nikolay Atanasov, Jerome Le Ny, Kostas Daniilidis, and George J. Pappas. Information acquisition with sensing robots: Algorithms and error bounds. In Proceedings of IEEE International Conference on Robotics and Automation, pages 6447-6454, Hong Kong, China, 2014.

[5] Daniel Axehill, Thomas Besselmann, Davide Martino Raimondo, and Manfred Morari. A parametric branch and bound approach to suboptimal explicit hybrid MPC. Automatica, 50(4):240-246, 2014.

[6] Yaakov Bar-Shalom, Peter K. Willett, and Xin Tian. Tracking and data fusion: A handbook of algorithms. YBS publishing, Storrs, CT, USA, 2011.

[7] Mokhtar S. Bazaraa, Hanif D. Sherali, and Chitharanjan M. Shetty. Nonlinear programming: theory and algorithms. John Wiley \& Sons, 2013.

[8] Richard E. Bellman. Dynamic Programming. Princeton University Press, Princeton, NJ, USA, 1957.

[9] Alberto Bemporad and Manfred Morari. Control of systems integrating logic, dynamics, and constraints. Automatica, 35(3):407 - 427, 1999.

[10] Kristoffer Bergman and Daniel Axehill. Combining homotopy methods and numerical optimal control to solve motion planning problems. In Proceedings of the IEEE Intelligent Vehicles Symposium, pages 347-354, Chang Shu, China, 2018. 
[11] Dimitri P. Bertsekas. Dynamic programming and optimal control, volume 1. Athena Scientific, Belmont, MA, USA, 2005.

[12] Dimitri P. Bertsekas. Nonlinear Programming. Athena Scientific, Belmont, MA, USA, 2016.

[13] John T. Betts. Practical methods for optimal control and estimation using nonlinear programming. SIAM, Philadelphia, PA, USA, 2010.

[14] Samuel Blackman and Robert Popoli. Design and Analysis of Modern Tracking Systems. Artech house, Norwood, MA, USA, 1999.

[15] Per Boström-Rost, Daniel Axehill, and Gustaf Hendeby. Informative path planning in the presence of adversarial observers. Accepted for publication in Proceedings of the International Conference on Information Fusion (FUSION), Ottawa, Canada, 2019.

[16] Per Boström-Rost, Daniel Axehill, and Gustaf Hendeby. On global optimization for informative path planning. IEEE Control Systems Letters, 2(4):833$838,2018$.

[17] Per Boström-Rost, Daniel Axehill, and Gustaf Hendeby. Informative path planning for active tracking of agile targets. In Proceedings of IEEE Aerospace Conference, Big Sky, MT, USA, 2019.

[18] Stephen Boyd and Lieven Vandenberghe. Convex Optimization. Cambridge University Press, Cambridge, UK, 2004.

[19] Stephen Boyd, Laurent El Ghaoui, Eric Feron, and Venkataramanan Balakrishnan. Linear matrix inequalities in system and control theory. SIAM, Philadelphia, PA, USA, 1994.

[20] Christof Büskens and Dennis Wassel. The ESA NLP solver WORHP. In Modeling and optimization in space engineering, pages 85-110. Springer, 2012.

[21] Thomas M. Cover and Joy A. Thomas. Elements of Information Theory. John Wiley \& Sons, Hoboken, NJ, USA, 2006.

[22] Matthew Dunbabin and Lino Marques. Robots for environmental monitoring: Significant advancements and applications. IEEE Robotics and Automation Magazine, 19(1):24-39, 2012.

[23] Christodoulos A. Floudas. Nonlinear and mixed-integer optimization: Fundamentals and applications. Oxford University Press, London, UK, 1995.

[24] Gene H. Golub and Charles F. Van Loan. Matrix Computations (3rd Ed.). Johns Hopkins University Press, Baltimore, MD, USA, 1996. ISBN 0-80185414-8.

[25] Peter E. Hart, Nils J. Nilsson, and Bertram Raphael. A formal basis for the heuristic determination of minimum cost paths. IEEE Transactions on Systems Science and Cybernetics, 4(2):100-107, 1968. 
[26] Gustaf Hendeby and Fredrik Gustafsson. Fundamental filtering limitations in linear non-Gaussian systems. In Proceedings of the 16th Triennial IFAC World Congress, pages 1209-1220, Prague, Czech Republic, 2005.

[27] Alfred O. Hero and Douglas Cochran. Sensor management: Past, present, and future. IEEE Sensors Journal, 11(12):3064-3075, 2011.

[28] Geoffrey A. Hollinger and Gaurav S. Sukhatme. Sampling-based robotic information gathering algorithms. The International Journal of Robotics Research, 33(9):1271-1287, 2014.

[29] Boris Houska, Hans Joachim Ferreau, and Moritz Diehl. ACADO Toolkit An open source framework for automatic control and dynamic optimization. Optimal Control Applications and Methods, 32(3):298-312, 2011.

[30] Marco Huber. Probabilistic framework for sensor management. PhD thesis, Univerität Karlsruhe (TH), 2009.

[31] Andrew H. Jazwinski. Stochastic Processes and Filtering Theory. Academic Press, New York, NY, USA, 1970.

[32] Siddharth Joshi and Stephen Boyd. Sensor selection via convex optimization. IEEE Transactions on Signal Processing, 57(2):451-462, 2009.

[33] Simon J. Julier. The scaled unscented transformation. In Proceedings of American Control Conference, pages 4555-4559, Anchorage, AK, USA, 2002.

[34] Thomas Kailath, Ali H. Sayed, and Babak Hassibi. Linear Estimation. Prentice Hall, Upper Saddle River, NJ, USA, 2000.

[35] Rudolph E. Kalman. A new approach to linear filtering and prediction problems. Transactions of the ASME, Journal of Basic Engineering, 82(1):35-45, 1960 .

[36] Sertac Karaman and Emilio Frazzoli. Sampling-based algorithms for optimal motion planning. The International Journal of Robotics Research, 30(7):846$894,2011$.

[37] Vijay Kumar, Daniela Rus, and Sanjiv Singh. Robot and sensor networks for first responders. IEEE Pervasive computing, 3(4):24-33, 2004.

[38] Xiaodong Lan and Mac Schwager. Rapidly exploring random cycles: Persistent estimation of spatiotemporal fields with multiple sensing robots. IEEE Transactions on Robotics, 32(5):1230-1244, 2016.

[39] Steven M. LaValle. Planning Algorithms. Cambridge University Press, Cambridge, UK, 2006.

[40] Steven M. LaValle and James J. Kuffner Jr. Randomized kinodynamic planning. The International Journal of Robotics Research, 20(5):378-400, 2001. 
[41] Jerome Le Ny and George J. Pappas. On trajectory optimization for active sensing in Gaussian process models. In Proceedings of the 48th IEEE Conference on Decision and Control, pages 6286-6292, Shanghai, China, 2009.

[42] Velodyne Lidar. Puck LITE. URL https://velodynelidar.com/ vlp-16-lite.html. Accessed: May 3rd, 2019.

[43] Maxim Likhachev, Geoffrey J. Gordon, and Sebastian Thrun. ARA*: Anytime A* with provable bounds on sub-optimality. In Advances in Neural Information Processing Systems 16, pages 767-774. MIT Press, 2004.

[44] Oskar Ljungqvist, Niclas Evestedt, Marcello Cirillo, Daniel Axehill, and Olov Holmer. Lattice-based motion planning for a general 2-trailer system. In Proceedings of the IEEE Intelligent Vehicles Symposium, pages 819-824, 2017.

[45] Johan Löfberg. YALMIP: A toolbox for modeling and optimization in MATLAB. In Proceedings of the CACSD Conference, Taipei, Taiwan, 2004.

[46] Jan Marian Maciejowski. Predictive control: With constraints. Prentice Hall, Englewood Cliffs, NJ, USA, 2002.

[47] Ronald Mahler. Objective functions for Bayesian control-theoretic sensor management, I: Multitarget first-moment approximation. In Proceedings of IEEE Aerospace Conference, pages 1905-1923, Big Sky, MT, USA, 2003.

[48] Salvatore Manfreda, Matthew McCabe, Pauline Miller, Richard Lucas, Victor Pajuelo Madrigal, Giorgos Mallinis, Eyal Ben Dor, David Helman, Lyndon Estes, Giuseppe Ciraolo, et al. On the use of unmanned aerial systems for environmental monitoring. Remote sensing, 10(4):641, 2018.

[49] Lewis Meier, John Peschon, and Robert Dressler. Optimal control of measurement subsystems. IEEE Transactions on Automatic Control, 12(5):528-536, 1967 .

[50] Jorge Nocedal and Stephen J. Wright. Numerical Optimization. Springer, New York, NY, USA, 2006.

[51] Brian Paden, Michal Čáp, Sze Zheng Yong, Dmitry Yershov, and Emilio Frazzoli. A survey of motion planning and control techniques for self-driving urban vehicles. IEEE Transactions on Intelligent Vehicles, 1(1):33-55, 2016.

[52] Sachin Patil, Yan Duan, John Schulman, Ken Goldberg, and Pieter Abbeel. Gaussian belief space planning with discontinuities in sensing domains. In Proceedings of IEEE International Conference on Robotics and Automation, pages 6483-6490, Hong Kong, China, 2014.

[53] Sachin Patil, Gregory Kahn, Michael Laskey, John Schulman, Ken Goldberg, and Pieter Abbeel. Scaling up Gaussian belief space planning through covariance-free trajectory optimization and automatic differentiation. In Algorithmic Foundations of Robotics XI, pages 515-533. Springer, Cham, Switzerland, 2015. 
[54] Judea Pearl. Heuristics: Intelligent search strategies for computer problem solving. Addison-Wesley Longman Publishing Co., Inc., Boston, MA, USA, 1984 .

[55] Mihail Pivtoraiko and Alonzo Kelly. Kinodynamic motion planning with state lattice motion primitives. In Proceedings of the IEEE/RSJ International Conference on Intelligent Robots and Systems, pages 2172-2179, San Francisco, CA, USA, 2011.

[56] Mihail Pivtoraiko, Ross A. Knepper, and Alonzo Kelly. Differentially constrained mobile robot motion planning in state lattices. Journal of Field Robotics, 26(3):308-333, 2009.

[57] Robert Platt Jr, Russ Tedrake, Leslie Kaelbling, and Tomas Lozano-Perez. Belief space planning assuming maximum likelihood observations. In Proceedings of Robotics: Science and Systems VI, Zaragoza, Spain, 2010.

[58] Samuel Prentice and Nicholas Roy. The belief roadmap: Efficient planning in belief space by factoring the covariance. The International Journal of Robotics Research, 28(11-12):1448-1465, 2009.

[59] Friedrich Pukelsheim. Optimal design of experiments. John Wiley \& Sons, New York, NY, USA, 1993.

[60] Arthur Richards, Tom Schouwenaars, Jonathan P. How, and Eric Feron. Spacecraft trajectory planning with avoidance constraints using mixed-integer linear programming. Journal of Guidance, Control, and Dynamics, 25(4):755$764,2002$.

[61] Christian P. Robert and George Casella. Monte Carlo statistical methods. Springer-Verlag, New York, NY, USA, 1999.

[62] Venkat Roy, Andrea Simonetto, and Geert Leus. Spatio-temporal sensor management for environmental field estimation. Signal Processing, 128:369$381,2016$.

[63] Brent Schlotfeldt, Dinesh Thakur, Nikolay Atanasov, Vijay Kumar, and George J. Pappas. Anytime planning for decentralized multirobot active information gathering. IEEE Robotics and Automation Letters, 3(2):1025-1032, 2018 .

[64] Amarjeet Singh, Andreas Krause, Carlos Guestrin, William J. Kaiser, and Maxim A. Batalin. Efficient planning of informative paths for multiple robots. In Proceedings of AAAI International Joint Conference on Artificial Intelligence, pages 2204-2211, Hyderabad, India, 2007.

[65] Bruno Sinopoli, Luca Schenato, Massimo Franceschetti, Kameshwar Poolla, Michael I. Jordan, and Shankar S. Sastry. Kalman filtering with intermittent observations. IEEE Transactions on Automatic Control, 49(9):1453-1464, 2004 . 
[66] Stephen L. Smith, Mac Schwager, and Daniela Rus. Persistent robotic tasks: Monitoring and sweeping in changing environments. IEEE Transactions on Robotics, 28(2):410-426, 2012.

[67] Simo Särkkä. Bayesian filtering and smoothing, volume 3. Cambridge University Press, Cambridge, UK, 2013.

[68] Sebastian Thrun, Wolfram Burgard, and Dieter Fox. Probabilistic robotics. MIT press, Cambridge, MA, USA, 2005.

[69] Jur Van Den Berg, Sachin Patil, and Ron Alterovitz. Motion planning under uncertainty using iterative local optimization in belief space. The International Journal of Robotics Research, 31(11):1263-1278, 2012.

[70] Michael P. Vitus, Wei Zhang, Alessandro Abate, Jianghai Hu, and Claire J. Tomlin. On efficient sensor scheduling for linear dynamical systems. Automatica, 48(10):2482-2493, 2012.

[71] H. Paul Williams. Model building in mathematical programming. John Wiley \& Sons, New York, NY, USA, 2013.

[72] Laurence A. Wolsey. Integer programming. John Wiley \& Sons, New York, NY, USA, 1998.

[73] Andreas Wächter and Lorenz T. Biegler. On the implementation of an interior-point filter line-search algorithm for large-scale nonlinear programming. Mathematical programming, 106(1):25-57, 2006.

[74] Chun Yang, Lance Kaplan, and Erik Blasch. Performance measures of covariance and information matrices in resource management for target state estimation. IEEE Transactions on Aerospace and Electronic Systems, 48(3): 2594-2613, 2012.

[75] Fumin Zhang and Naomi Ehrich Leonard. Cooperative filters and control for cooperative exploration. IEEE Transactions on Automatic Control, 55(3): 650-663, 2010.

[76] Karl J. Åström. Introduction to stochastic control theory. Academic Press, New York, NY, USA, 1970. 


\section{Licentiate Theses \\ Division of Automatic Control \\ Linköping University}

P. Andersson: Adaptive Forgetting through Multiple Models and Adaptive Control of Car Dynamics. Thesis No. 15, 1983.

B. Wahlberg: On Model Simplification in System Identification. Thesis No. 47, 1985.

A. Isaksson: Identification of Time Varying Systems and Applications of System Identification to Signal Processing. Thesis No. 75, 1986.

G. Malmberg: A Study of Adaptive Control Missiles. Thesis No. 76, 1986.

S. Gunnarsson: On the Mean Square Error of Transfer Function Estimates with Applications to Control. Thesis No. 90, 1986.

M. Viberg: On the Adaptive Array Problem. Thesis No. 117, 1987.

K. Ståhl: On the Frequency Domain Analysis of Nonlinear Systems. Thesis No. 137, 1988.

A. Skeppstedt: Construction of Composite Models from Large Data-Sets. Thesis No. 149, 1988.

P. A. J. Nagy: MaMiS: A Programming Environment for Numeric/Symbolic Data Processing. Thesis No. 153, 1988.

K. Forsman: Applications of Constructive Algebra to Control Problems. Thesis No. 231, 1990.

I. Klein: Planning for a Class of Sequential Control Problems. Thesis No. 234, 1990.

F. Gustafsson: Optimal Segmentation of Linear Regression Parameters. Thesis No. 246, 1990.

H. Hjalmarsson: On Estimation of Model Quality in System Identification. Thesis No. 251, 1990.

S. Andersson: Sensor Array Processing; Application to Mobile Communication Systems and Dimension Reduction. Thesis No. 255, 1990.

K. Wang Chen: Observability and Invertibility of Nonlinear Systems: A Differential Algebraic Approach. Thesis No. 282, 1991.

J. Sjöberg: Regularization Issues in Neural Network Models of Dynamical Systems. Thesis No. 366, 1993.

P. Pucar: Segmentation of Laser Range Radar Images Using Hidden Markov Field Models. Thesis No. 403, 1993.

H. Fortell: Volterra and Algebraic Approaches to the Zero Dynamics. Thesis No. 438, 1994.

T. McKelvey: On State-Space Models in System Identification. Thesis No. 447, 1994.

T. Andersson: Concepts and Algorithms for Non-Linear System Identifiability. Thesis No. 448, 1994.

P. Lindskog: Algorithms and Tools for System Identification Using Prior Knowledge. Thesis No. 456, 1994.

J. Plantin: Algebraic Methods for Verification and Control of Discrete Event Dynamic Systems. Thesis No. 501, 1995.

J. Gunnarsson: On Modeling of Discrete Event Dynamic Systems, Using Symbolic Algebraic Methods. Thesis No. 502, 1995.

A. Ericsson: Fast Power Control to Counteract Rayleigh Fading in Cellular Radio Systems. Thesis No. 527, 1995.

M. Jirstrand: Algebraic Methods for Modeling and Design in Control. Thesis No. 540, 1996.

K. Edström: Simulation of Mode Switching Systems Using Switched Bond Graphs. Thesis No. 586, 1996. 
J. Palmqvist: On Integrity Monitoring of Integrated Navigation Systems. Thesis No. 600, 1997.

A. Stenman: Just-in-Time Models with Applications to Dynamical Systems. Thesis No. 601, 1997.

M. Andersson: Experimental Design and Updating of Finite Element Models. Thesis No. 611, 1997.

U. Forssell: Properties and Usage of Closed-Loop Identification Methods. Thesis No. 641, 1997.

M. Larsson: On Modeling and Diagnosis of Discrete Event Dynamic systems. Thesis No. 648, 1997.

N. Bergman: Bayesian Inference in Terrain Navigation. Thesis No. 649, 1997.

V. Einarsson: On Verification of Switched Systems Using Abstractions. Thesis No. 705, 1998.

J. Blom, F. Gunnarsson: Power Control in Cellular Radio Systems. Thesis No. 706, 1998.

P. Spångéus: Hybrid Control using LP and LMI methods - Some Applications. Thesis No. 724, 1998.

M. Norrlöf: On Analysis and Implementation of Iterative Learning Control. Thesis No. 727, 1998.

A. Hagenblad: Aspects of the Identification of Wiener Models. Thesis No. 793, 1999.

F. Tjärnström: Quality Estimation of Approximate Models. Thesis No. 810, 2000.

C. Carlsson: Vehicle Size and Orientation Estimation Using Geometric Fitting. Thesis No. 840, 2000.

J. Löfberg: Linear Model Predictive Control: Stability and Robustness. Thesis No. 866, 2001.

O. Härkegård: Flight Control Design Using Backstepping. Thesis No. 875, 2001.

J. Elbornsson: Equalization of Distortion in A/D Converters. Thesis No. 883, 2001.

J. Roll: Robust Verification and Identification of Piecewise Affine Systems. Thesis No. 899, 2001.

I. Lind: Regressor Selection in System Identification using ANOVA. Thesis No. 921, 2001.

R. Karlsson: Simulation Based Methods for Target Tracking. Thesis No. 930, 2002.

P.-J. Nordlund: Sequential Monte Carlo Filters and Integrated Navigation. Thesis No. 945, 2002.

M. Östring: Identification, Diagnosis, and Control of a Flexible Robot Arm. Thesis No. 948, 2002.

C. Olsson: Active Engine Vibration Isolation using Feedback Control. Thesis No. 968, 2002 .

J. Jansson: Tracking and Decision Making for Automotive Collision Avoidance. Thesis No. 965, 2002.

N. Persson: Event Based Sampling with Application to Spectral Estimation. Thesis No. 981, 2002.

D. Lindgren: Subspace Selection Techniques for Classification Problems. Thesis No. 995, 2002.

E. Geijer Lundin: Uplink Load in CDMA Cellular Systems. Thesis No. 1045, 2003.

M. Enqvist: Some Results on Linear Models of Nonlinear Systems. Thesis No. 1046, 2003.

T. Schön: On Computational Methods for Nonlinear Estimation. Thesis No. 1047, 2003.

F. Gunnarsson: On Modeling and Control of Network Queue Dynamics. Thesis No. 1048, 2003.

S. Björklund: A Survey and Comparison of Time-Delay Estimation Methods in Linear Systems. Thesis No. 1061, 2003. 
M. Gerdin: Parameter Estimation in Linear Descriptor Systems. Thesis No. 1085, 2004.

A. Eidehall: An Automotive Lane Guidance System. Thesis No. 1122, 2004.

E. Wernholt: On Multivariable and Nonlinear Identification of Industrial Robots. Thesis No. 1131, 2004.

J. Gillberg: Methods for Frequency Domain Estimation of Continuous-Time Models. Thesis No. 1133, 2004.

G. Hendeby: Fundamental Estimation and Detection Limits in Linear Non-Gaussian Systems. Thesis No. 1199, 2005.

D. Axehill: Applications of Integer Quadratic Programming in Control and Communication. Thesis No. 1218, 2005.

J. Sjöberg: Some Results On Optimal Control for Nonlinear Descriptor Systems. Thesis No. 1227, 2006.

D. Törnqvist: Statistical Fault Detection with Applications to IMU Disturbances. Thesis No. 1258, 2006.

H. Tidefelt: Structural algorithms and perturbations in differential-algebraic equations. Thesis No. 1318, 2007.

S. Moberg: On Modeling and Control of Flexible Manipulators. Thesis No. 1336, 2007.

J. Wallén: On Kinematic Modelling and Iterative Learning Control of Industrial Robots. Thesis No. 1343, 2008.

J. Harju Johansson: A Structure Utilizing Inexact Primal-Dual Interior-Point Method for Analysis of Linear Differential Inclusions. Thesis No. 1367, 2008.

J. D. Hol: Pose Estimation and Calibration Algorithms for Vision and Inertial Sensors. Thesis No. 1370, 2008.

H. Ohlsson: Regression on Manifolds with Implications for System Identification. Thesis No. 1382, 2008.

D. Ankelhed: On low order controller synthesis using rational constraints. Thesis No. 1398, 2009.

P. Skoglar: Planning Methods for Aerial Exploration and Ground Target Tracking. Thesis No. 1420, 2009.

C. Lundquist: Automotive Sensor Fusion for Situation Awareness. Thesis No. 1422, 2009.

C. Lyzell: Initialization Methods for System Identification. Thesis No. 1426, 2009.

R. Falkeborn: Structure exploitation in semidefinite programming for control. Thesis No. 1430, 2010.

D. Petersson: Nonlinear Optimization Approaches to $\mathcal{H}_{2}$-Norm Based LPV Modelling and Control. Thesis No. 1453, 2010.

Z. Sjanic: Navigation and SAR Auto-focusing in a Sensor Fusion Framework. Thesis No. 1464, 2011.

K. Granström: Loop detection and extended target tracking using laser data. Thesis No. 1465, 2011.

J. Callmer: Topics in Localization and Mapping. Thesis No. 1489, 2011.

F. Lindsten: Rao-Blackwellised particle methods for inference and identification. Thesis No. 1480, 2011.

M. Skoglund: Visual Inertial Navigation and Calibration. Thesis No. 1500, 2011.

S. Khoshfetrat Pakazad: Topics in Robustness Analysis. Thesis No. 1512, 2011.

P. Axelsson: On Sensor Fusion Applied to Industrial Manipulators. Thesis No. 1511, 2011.

A. Carvalho Bittencourt: On Modeling and Diagnosis of Friction and Wear in Industrial Robots. Thesis No. 1516, 2012. 
P. Rosander: Averaging level control in the presence of frequent inlet flow upsets. Thesis No. 1527, 2012.

N. Wahlström: Localization using Magnetometers and Light Sensors. Thesis No. 1581, 2013.

R. Larsson: System Identification of Flight Mechanical Characteristics. Thesis No. 1599, 2013.

Y. Jung: Estimation of Inverse Models Applied to Power Amplifier Predistortion. Thesis No. 1605, 2013.

M. Syldatk: On Calibration of Ground Sensor Networks. Thesis No. 1611, 2013.

M. Roth: Kalman Filters for Nonlinear Systems and Heavy-Tailed Noise. Thesis No. 1613, 2013.

D. Simon: Model Predictive Control in Flight Control Design - Stability and Reference Tracking. Thesis No. 1642, 2014.

J. Dahlin: Sequential Monte Carlo for inference in nonlinear state space models. Thesis No. 1652, 2014.

M. Kok: Probabilistic modeling for positioning applications using inertial sensors. Thesis No. 1656, 2014.

J. Linder: Graybox Modelling of Ships Using Indirect Input Measurements. Thesis No. 1681, 2014.

G. Mathai: Direction of Arrival Estimation of Wideband Acoustic Wavefields in a Passive Sensing Environment. Thesis No. 1721, 2015.

I. Nielsen: On Structure Exploiting Numerical Algorithms for Model Predictive Control. Thesis No. 1727, 2015.

C. Veibäck: Tracking of Animals Using Airborne Cameras. Thesis No. 1761, 2016.

N. Evestedt: Sampling Based Motion Planning for Heavy Duty Autonomous Vehicles. Thesis No. 1762, 2016.

H. Nyqvist: On Pose Estimation in Room-Scaled Environments. Thesis No. 1765, 2016.

Y. Zhao: Position Estimation in Uncertain Radio Environments and Trajectory Learning. Thesis No. 1772, 2017.

P. Kasebzadeh: Parameter Estimation for Mobile Positioning Applications. Thesis No. 1786, 2017.

K. Radnosrati: On Timing-Based Localization in Cellular Radio Networks. Thesis No. 1808, 2018.

G. Lindmark: Methods and Algorithms for Control Input Placement in Complex Networks. Thesis No. 1814, 2018.

M. Lindfors: Frequency Tracking for Speed Estimation. Thesis No. 1815, 2018.

D. Ho: Some results on closed-loop identification of quadcopters. Thesis No. 1826, 2018.

O. Ljungqvist: On motion planning and control for truck and trailer systems. Thesis No. 1832, 2019. 


\section{FACULTY OF SCIENCE AND ENGINEERING}

Linköping studies in science and technology. Licentiate Thesis No. 1838

Department of Electrical Engineering

Linköping University

SE-581 83 Linköping, Sweden

www.liu.se 